RACHEL CAMPBELL

\title{
Rethinking Risk
}

\section{in International Financial Markets}




\section{RETHINKING RISK IN INTERNATIONAL FINANCIAL MARKETS}

Rachel Campbell 


\section{EEN NIEUWE KIJK OP RISICO}

IN INTERNATIONALE FINANCIÀLE MARKTEN

\section{PROEFSCHRIFT}

TER VERKRIJGING VAN DE GRAAD VAN DOCTOR AAN DE ERASMUS UNIVERSITEIT ROTTERDAM OP GEZAG VAN DE RECTOR MAGNIFICUS

PROF.DR.IR. J.H. VAN BEMMEL

EN VOLGENS BESLUIT VAN HET COLLEGE VOOR PROMOTIES

DE OPENBARE VERDEDIGING ZAL PLAATSVINDEN OP

VRIJDAG 7 SEPTEMBER 2001 OM 13.30 UUR

DOOR

RACHEL ANN JANE CAMPBELL (POWNALL)

GEBOREN OP 28 DECEMBER 1973 


\section{Promotiecommissie}

Promotor:

Prof. dr. C.G. Koedijk

Overige leden:

Prof. dr. C. de Vries

Prof. dr. J. Spronk

Prof. dr. ir. A. Smidts

Erasmus Research Institute of Management (ERIM)

Erasmus University Rotterdam

Internet: www.erim.eur.nl

ERIM Ph.D. series Research in Management 5

ISBN 90-5892-008-9

All rights reserved. No part of this publication may be reproduced or transmitted in any form or by any means electronic or mechanical, including photocopying, recording, or by any information storage and retrieval system, without permission in writing from the author(s). 


\section{ACKNOWLEDGEMENTS}

I would like to thank all those who have helped me whilst undertaking the research for my doctoral thesis. My gratitude goes in particular to my family and friends, all those at the Finance and Accounting department at Maastricht University, and the Financial Management Department at the Erasmus University, Rotterdam. Without whose support I would not have been able to complete this work. I would also like to thank the NWO for the grant which supported the research. I have very much enjoyed the time spent during my Ph.D. both in Maastricht and in Rotterdam. It has not only allowed me to delve further into the current academic literature in international finance but also to broaden my horizonsÖ thanksÖ 
Naturally devoted to Chris 


\section{FOREWORD}

\section{CHAPTER 1 Introduction}

\section{CHAPTER 2 A Downside Risk Framework for Optimal} Portfolio Selection

2.1

2.2

Portfolio Selection under Shortfall Constraints

2.2.1

2.2 .2

2.3 Portfolio Selection Model

2.3.1

2.3.2

2.4

Portfolio Selection Problem and Downside Risk

Constraint

Optimal Portfolio Construction

Optimal Portfolio Selection for US Stocks and Bonds Empirical Distribution

Alternative Parametric Distributions

Concluding Remarks

\section{CHAPTER 3 Fat Tails in Financial Risk Management}

3.1

3.1 .1

3.1 .2

3.1 .3

3.1 .4

3.2

3.3

3.4
Value-at-Risk Methodology

Methods to Estimate the Cutoff Return and VaR

Empirical VaR

Normal VaR

VaR-x

Data

Value-at-Risk Estimates

Concluding Remarks 
CHAPTER 4 Measuring Credit Spread Risk: Incorporating the Tails

$4.1 \quad$ Credit Spreads

4.2

4.3

4.4

Historical Credit Spread Widenings

Tail Index Estimation

Concluding Remarks

CHAPTER 5 Times of Crisis: A Conditional or Unconditional Approach?

5.1

Risk Management in Asian Financial Markets

5.1 .1

5.1 .2

5.2

Unconditional Value-at-Risk

RiskMetrics ${ }^{\text {TM }}$

5.2 .1

5.2 .2

Downside Risk and Financial Crises

Tail Index Estimation

5.3

Conditional VaR-x

5.4

Implications for Portfolio Selection

Concluding Remarks

\section{CHAPTER 6 Covariance and Correlation}

6.1

Alternative Conditional Correlation Structure

6.2

6.3

International Equity Markets

6.4

Implications for Portfolio Diversification

Concluding Remarks 


\begin{tabular}{|c|c|}
\hline CHAPTER 7 & $\begin{array}{l}\text { The Downside of International Equity } \\
\text { Markets }\end{array}$ \\
\hline 7.1 & Pricing Financial Assets \\
\hline 7.1.1 & Consumption-Based Approach \\
\hline 7.1.1.1 & Equity Premium Puzzle \\
\hline 7.1.1.2 & Home-Bias Phenomenon \\
\hline 7.1.2 & Behavourial Finance \\
\hline 7.1.3 & Downside Risk \\
\hline 7.2 & Optimal Asset Allocation under Downside Risk \\
\hline 7.2.1 & Data \\
\hline 7.2.2 & Empirical Results for US Domestic Market \\
\hline 7.2 .3 & Empirical Results for International Equity Mark \\
\hline 7.3 & Concluding Remarks \\
\hline
\end{tabular}

\section{CHAPTER 8 Concluding Thoughts}

CHAPTER 9 References

CHAPTER 10 Summary in Dutch / Nederlandse Samenvatting 


\section{CHAPTER 1 INTRODUCTION}

International financial markets are inherently risky places for investors and international financial corporations alike. Changes in exchange rates, interest rates, equity prices, and real estate all have a profound affect on national economies by dramatically influencing the profits made by international companies as well as the returns made by institutional and individual investors. Exactly how much an individual or institution is affected depends of course on the degree of exposure to the market. Many investment banks and institutions lost millions due to ëBlack Mondayí on Wall Street, when the Dow Jones Average fell 508.32 points, a drop of $22.6 \%$, the largest drop in both size and volume since 1914 . On ëBlack Wednesdayí in September 1992 those with long positions on the British Pound also made huge losses when it fell and Britain was suddenly forced to leave the European Exchange Rate Mechanism. In December 1994, the Mexican Peso fell dramatically when capital fled out of the country, resulting in liquidity drying up. The so-called Tequila effect resulted, with investors consequently shying away from other Latin American economies, such as those of Argentina and Brazil. The fall of the Baht in Thailand was the spark that caused the eruption of the Asian financial crisis, hitting most of the East Asian markets in mid-1997 and resulting in currencies, stock markets, and other asset prices falling to early 
1990 ís levels. Russia defaulted on its huge foreign debts in August 1998, causing further turbulence in other emerging markets. Exposure to any of these fairly recent financial crises would have had detrimental effects on the portfolio value of many institutions and individuals, resulting in millions of dollars being lost. In the aftermath of these financial crises many financial markets were thought to be more risky than previously perceived, with investors shying away from these markets or where necessary improving their hedging strategies to exposure on international financial markets.

To control movements in international financial markets to avoid such losses is impossible. However risk exposure from positions may be managed by buying insurance to protect against adverse market movements and thus reducing exposure. The development of risk management products, such as derivatives, means that a third party can be paid to take on a companyís or investorís unwanted exposure, without having to close out the position completely. This means that a choice can be made as to the extent of the risk held by the investor or institution and additional unwanted exposure may be hedged. This should result in investors and institutions bearing only the risk that they are prepared to face - reducing their exposure to unwanted risks. For example an airline company exposed to changing demand for air travel, faces the risk of making large loses when demand is low. It also faces the risk of high fuel costs, and therefore is exposed to the commodity risk on the price of oil. To hedge against this risk, and bear only the risk of the core business for air travel, the airline company could choose to hedge its exposure to increasing oil prices by buying derivatives contracts on oil. These act like an insurance policy, and can serve to lock in the futures price for oil, so that the airline is no longer exposed to large negative movements in oil prices. Likewise a European investor for example investing in Latin 


\section{Chapter 1: Introduction}

American equities is exposed to both the market risk of a drop in the price of equities and to an adverse movement in exchange rates. The investor can hedge either of these positions so that the degree to which there is exposure to risk may be chosen. Managing risk should therefore enable a better strategic decision on the type and size of risks faced.

The advent of risk management products and hedging strategies has however also meant greater leveraging on positions and additional speculative risk being taken on. Some risk management products originally developed to improve risk management have therefore led to the downfall of many companies through improper use of derivative instruments and speculative rather than hedged positions being taken. The collapse of Barings, as well as the scandals affecting many investment banks and institutions, such as Daiwa, Sumitomo Corp., Credit Lyonnais, Orange county, Metallgeschellschaft, Morgan Grenfell, NatWest Markets, and Lloyd's of London, are just examples of the financial difficulties resulting from fraudulent and speculative dealings in derivatives.

It is not just the companies themselves who have been affected by recent turmoil in financial markets. The indirect effects of financial market fluctuations also bear heavily on the lives of the average man or woman on the street. Money tied up in life insurance policies or pension funds whose value depends on the movement of stock and bond prices. House prices depend on the real estate market and the term structure of interest rates. Purchasing power whilst on holiday abroad and the strength of the domestic economy depends on current exchange rates. The market value of these policies, stocks, real estate and exchange rates, all depend on the premium paid for bearing the risk of uncertainty regarding the future value of the asset held. Of course this price depends on the average 
investorís appetite towards risk. Exactly how much investors are willing to pay for risky stocks depends on how the investor values the potential high reward that can be earned for bearing the risk. But the risk can only be priced when we know exactly what type of risk investors face from exposure to the various international financial markets. Determining what contributes to the risk we actually face from holding assets in international financial markets is the core of finance theory and is central to this thesis.

To enable a better understanding of the risks inherent in financial markets, we first take a look at the performance of a variety of financial assets in the US in recent years. We employ monthly historical data on equities, corporate and government bonds for various maturities over the past 20 years. It is interesting to compare their return performances over the period, to give us an idea of the extent of the premium earned for bearing additional risk from exposure to fluctuations in the prices of the various financial assets. In general we would expect that only if a premium is earned in terms of higher returns on financial assets will investors be induced to take on the risk of holding more risky assets. Short term Treasury-bills are regarded as the least risky of the various assets. There is hardly any risk of default. They guarantee a certain payoff at a future date in time, so that the risk to the holder is the risk from inflation, since the return is a nominal rather than a real return. The 3-Month T-bill is therefore commonly used as a proxy for the riskfree rate. Long-term Government bonds have the additional risk of price changes, since the bondís yield fluctuates according to changes in interest rates, so that if the bond is not held until maturity the holder bears some price risk. However on maturity the bond has par value and hence the return is known at the purchase time, so that at all points in time the future return is known. Corporate bonds however have the additional risk of default over government 


\section{Chapter 1: Introduction}

bonds, since there is the likelihood that the institution writing the bonds may default on payment of the bond at maturity. The lower the credit rating then the higher this potential risk to the holder of the bond.

\section{Figure 1.1}

\section{US Indices 1980 ñ 2000}

The figure presents the performance of the $S \& P 500$, the $S \& P$ small caps, Salomon Brothers Aaa and Baa rated Corporate Bond Indices, 30-Year and 2-Year Government Bonds, and the 3-Month Treasury bill. We use monthly data over the period 1980-2000.

US Indices: Monthly Data 1980-2000

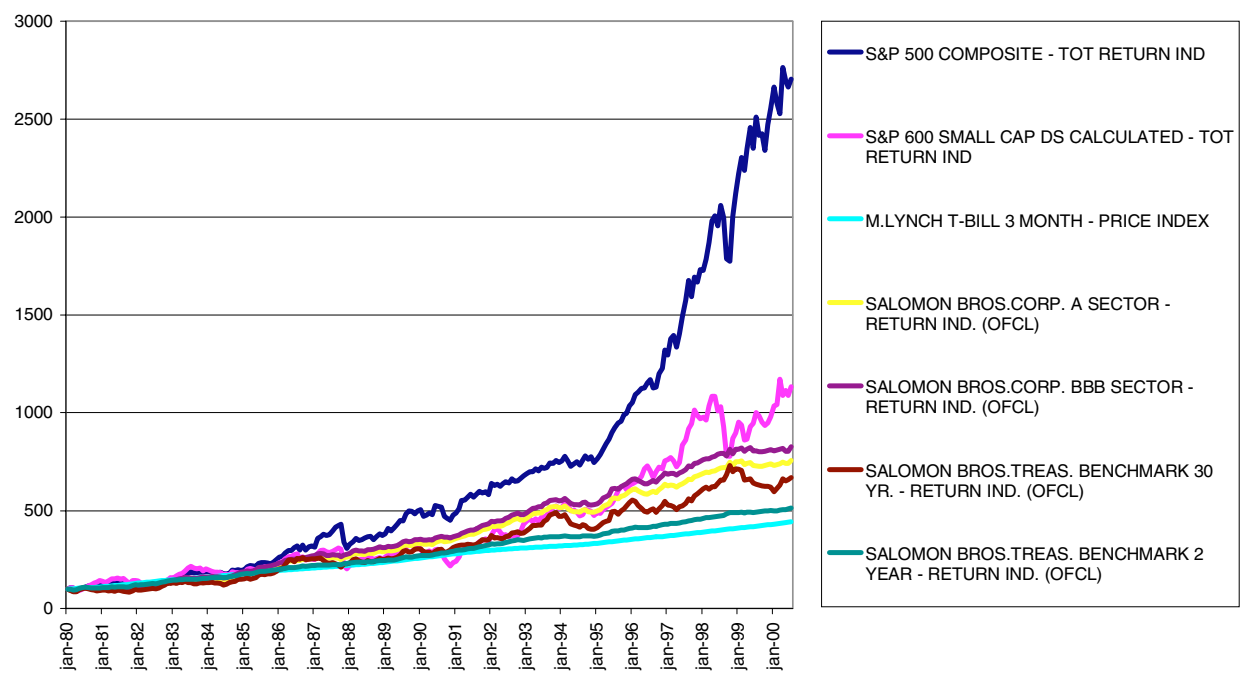

The Stock Market Index for the US, the S\&P500, is regarded as much more risky than bond indices. Common stocks represent a share in a corporation or enterprise so that the return depends on the fortunes of the firm itself, also dependent on overall market and 
Chapter 1: Introduction

economic conditions. The stock market index represents a weighted average of shares, so that any individual business risk is thought to be diversified away. Small caps represent only the percentage of the market for which firms have a small market capitalisation. They are therefore thought to bear additional liquidity risk since they are less frequently traded. However over the past 20 years they have not earned a premium over the S\&P 500 . We can see from figure 1.1 how the return on the S\&P500 has been much greater than on corporate bonds or government bonds, and that an investment in the 3month T-bill would only have generated a return in line with inflation.

In an efficient market the additional risk from holding a financial asset should be correctly priced, so that the premium for risk depends directly on the extent of the risk born by the investor. It should therefore be the case that those assets bearing the greatest risk to the investor, should also on average receive the highest returns. It is easy to measure the extent of the risk premium (the excess return over the risk free rate). However just how do we measure the additional risk involved in holding the S\&P500 instead of corporate bonds? Is this risk worth bearing? And if so how much risk should one face? These questions are core in the theory of optimal portfolio selection and are also at the heart of financial risk management. To determine whether the risk from exposure is worth taking it is first crucial to understand exactly what financial risks are involved when taking on a position in a financial asset. Or stated otherwise, do the rewards from bearing the risk compensate the level of risk borne? This enables us to answer the basic question in optimal asset allocation: how to optimise the level of return for a given amount of risk. Moreover active financial risk management involves how one can best reduce the risk for a given level of 


\section{Chapter 1: Introduction}

return so that the individual or institution is able to bear the risk of loss, in the case of the worst-case scenario actually happening.

However, exactly how we measure and estimate risk is still open to debate. It is all very well stating that stocks are more risky than bonds and that therefore the required rate of return needs to be higher on equities to induce the risk-averse investor to hold equities. However just how do we measure how much more risky equities are for example than bonds to account for the additional return? The exact size of the risk-return trade-off therefore depends crucially on how we estimate risk for the various asset classes. In modern portfolio theory, Markowitz (1954) defines risk as the deviation of returns from the expected return, variance, or its square root, standard deviation. This builds upon the assumption of normally distributed returns, so that the variance is able to capture all variation in the return distribution. It is this definition of risk, which is currently used in assessing risk-return trade-offs in financial markets and is behind the use of beta as a risk measure.

To see how Markowitzís definition of risk compares with the return premiums made in recent times, we have computed the risk-return trade-off using the standard deviation of the return distribution as the proxy for risk. The average annual return for the various assets over the period $1980-2000$, along with the risk premium for each asset, the standard deviation, and the Sharpe Index, (the risk premium divided by the standard deviation of the distribution) are given in Table 1.1. 
Table 1.1

\section{US Indices 1980:2000}

The table presents the performance of the S\&P 500, the S\&P small caps, Salomon Brothers Aaa and Baa rated Corporate Bond Indices, 30-Year and 2-Year Government Bonds, and the 3-Month Treasury bill. We use monthly data over the period 1980-2000.

\begin{tabular}{lcccc}
\hline & $\begin{array}{c}\text { Average } \\
\text { Annual } \\
\text { Return }\end{array}$ & $\begin{array}{c}\text { Risk } \\
\text { Premia }\end{array}$ & $\begin{array}{c}\text { Standard } \\
\text { Deviation }\end{array}$ & $\begin{array}{c}\text { Sharpe } \\
\text { Index }\end{array}$ \\
\hline S\&P 500 COMPOSITE & $17.28 \%$ & $9.93 \%$ & $14.31 \%$ & 0.6938 \\
$\begin{array}{l}\text { S\&P 600 SMALL CAP DS } \\
\text { CALCULATED }\end{array}$ & $13.92 \%$ & $6.57 \%$ & $18.17 \%$ & 0.3618 \\
$\begin{array}{l}\text { SALOMON BROS.CORP. } \\
\text { BBB SECTOR. (OFCL) }\end{array}$ & $10.74 \%$ & $3.39 \%$ & $7.35 \%$ & 0.4618 \\
$\begin{array}{l}\text { SALOMON BROS.CORP. A } \\
\text { SECTOR (OFCL) }\end{array}$ & $10.29 \%$ & $2.94 \%$ & $7.86 \%$ & 0.3745 \\
$\begin{array}{l}\text { SALOMON BROS.TREAS. } \\
\text { BENCHMARK 30 YR. }\end{array}$ & $9.70 \%$ & $2.36 \%$ & $12.21 \%$ & 0.1929 \\
(OFCL) & & & & \\
$\begin{array}{l}\text { SALOMON BROS.TREAS. } \\
\text { BENCHMARK 2 YEAR } \\
\text { OFCL) }\end{array}$ & $8.14 \%$ & $0.79 \%$ & $3.57 \%$ & 0.2206 \\
$\begin{array}{l}\text { M.LYNCH T-BILL 3 MONTH } \\
\text { - PRICE INDEX }\end{array}$ & $7.35 \%$ & $0.00 \%$ & $1.01 \%$ & \\
\hline
\end{tabular}

The return premium for holding equities over 3-month T-bills has for example been roughly $10 \%$ over the last 20 years in the US. This is commonly denoted as the risk premium and is higher than it has been in previous periods. The Sharpe index captures the ratio of the additional return earned on an asset over and above the T-bill rate to the 


\section{Chapter 1: Introduction}

variance of the return distribution, and is given in the final column of Table 1.1. If the standard deviation were able to capture all of the risk involved in investing in financial assets then the Sharpe index would be constant over all asset classes over a particular period in time. Of course the risk premium may not be stochastic, as has been the case over recent years, but in general the Sharpe index should give an indication of the risk-return trade-off in financial markets.

It would however appear that the premium earned by equities and by B grade corporate bonds is far greater than the premium on government bonds. This has been a puzzle to economists and is commonly termed the equity premium puzzle. Using the standard deviation as the appropriate measure for risk, the risk in investing in stocks appears to be very low given the phenomenal returns made in stocks over the past 20 years. To explain this equity risk premium investors would have had to have been extremely averse to risk, i.e. the premium for risk required to induce investors to hold stocks would have had to have been extremely large. Using a utility model to fit the equity premium generally requires an inconceivably large factor for the level of relative risk aversion, way beyond empirical estimates of investor risk aversion. A further puzzle is the home bias phenomenon. Using standard deviation as the measure for risk investors have appeared to shy away from prevailing international diversification benefits, holding a larger percentage of investment portfolios in domestic investments than current portfolio theory would suggest. It would appear that the risk of investing internationally is greater than currently captured by the standard deviation of returns. 
One trajectory for research in resolving many of these puzzles is through alternative measures for the definition of risk. It may be that risk is under-estimated and not fully accounted for. The risk of investing in stocks may in fact be much more risky than the standard deviation assumes. An alternative approach to Markowitzís mean-variance optimisation was developed in the same year by Roy in 1952, Safety First Theory. He pioneered the idea that investors maximise the return among only those assets whose probability of failure does not exceed a critical level. In doing so investors therefore perceive risk as the possibility of negative movements below a critical level, rather than only according to the standard deviation of returns. These two measures are indeed identical when the return distribution is normal. However non-normality in empirical data suggests the likelihood of alternative risk-return trade-offs using the two approaches. Further evidence against the current approach using expected utility theory is the contradicting results of empirical studies on investment behaviour. Expected utility theory is based on the axioms of van Neumann and Morgenstern, (1944), and three tenets: expectation, asset integration and risk aversion. These tenets have however been shown to be broken (Allais 1953, Williams 1966, Kahneman and Tversky 1979) with behaviour differing according to whether the investor is in the positive (gains) or negative (losses) domain for the value function. It would appear that losses loom larger than gains, loss aversion, and instead of treating the negative domain as equal to the positive domain it would be more appropriate to weigh the returns by a value function. This is the approach of Kahneman and Tversky in their pioneering work on Prospect Theory. They also find evidence of decisions depending on the extent of loses and gains being made with respect to a reference point; depending on the original state of being. Thus rather than looking at 


\section{Chapter 1: Introduction}

final states only, investors concern themselves about changes in wealth or welfare, opportunity costs.

In this thesis we aim to address many of the issues raised concerning the appropriate definition and measurement of risk. We investigate an alternative approach to the estimation of risk, and the risk-return trade-off in international financial markets. Rather than focussing on the deviation of returns as the only appropriate measure for risk, we focus on the more relevant negative domain when defining risk, and thus the notion of downside risk as the correct measure for risk. This was in fact Markowitzís original idea, proposing the use of semi-variance as the appropriate measure for risk, however computational difficulty led to the use of variance. We develop upon the pioneering ideas of Markowitz (1954), Roy (1954) and Kahneman and Tversky (1979), and hope to give some greater insight into the risk-return trade-offs faced in international financial markets.

In the following chapter, the first research chapter, we introduce the issue of downside risk for optimal portfolio theory. Investors are more concerned with losses than gains when making decisions on risk, and they are also thought to make them relative to a reference point. In developing the model we move away from the tenets of expected utility theory, and derive a model for optimal portfolio selection for an investor concerned with downside risk. Taking such an approach enables us to provide a general framework, without the need to resort to parametric assumptions about the overall return distribution. We build on the work of Roy (1952) and Arzac and Bawa (1977), and when returns are assumed to be normally distributed include Markowitzís mean-variance portfolio theory (1952) as a 
special case. We are therefore able to compare the effects of alternative parametric assumptions on the optimal portfolio.

In chapter three we focus more specifically on the correct estimation of downside risk and develop a model to improve market risk estimation. Empirical research on international financial market returns has shown that for many asset classes returns are both skewed and fat tailed. This means that there is a larger probability of negative returns than under the assumption of normality. This renders the assumption of normality as inappropriate for analysis of the tails of the return distribution, so that the use of variance alone as a measure for risk is incomplete. Using methods developed from Extreme Value Theory, we develop a model for estimating downside risk for market risk, which incorporates the additional probability of large loses occurring in international financial markets, VaR-x. In the chapter following, chapter four, we focus on credit risk, which also includes the risk from potential counterparty default. The larger the credit spread then the greater the credit risk on the product. Again the potential risk is greater than that captured by the variance of the distribution alone, so that we need to incorporate the additional downside risk of large widenings in credit spreads. We develop a model for improving the estimation of the risk of credit spread widenings and apply it to data on international swap markets.

In chapter five we turn to times of crisis. It is during periods of financial turmoil with the probability of large loses occurring being greater, that we are able to test definitions of risk. We develop a conditional approach to risk management for the VaR-x approach, and compare its performance to a current standard in risk management RiskMetrics ${ }^{\mathrm{TM}}$. We find that during times of crisis deviations from normality become more severe, so that the 


\section{Chapter 1: Introduction}

effects of incorrect measures for risk become more apparent. It would appear that the risk of extreme market movements in times of crisis is much larger than current finance theory leads us to believe; there is additional downside risk which needs to be incorporated into modern finance.

To further our understanding of correct risk measurement in chapter six we also investigate the possibility of conditional correlation structures. We are interested in seeing whether the correlation between markets for large movements is greater than for more common movements. If so the effects are crucial for portfolio management, since diversification benefits are less significant than previously thought. We develop a quantile correlation model that conditions on the size of the market movement, and compare the results to the previous approaches of Longin and Solnik (1995) and Boyer, Gibson and Loretan (1999).

In the final research chapter, chapter seven, we pool together the research of the previous five chapters, to incorporate the results of improved risk estimation on the downside risk portfolio model. Using additional data on international markets we compare the risk-return trade-offís for both US and international investors to provide an alternative view to how investorís perceive risk on financial markets. This gives some insight into how some of the current puzzles in the finance literature may be resolved through an alternative approach to the estimation of risk. Through the research on improving risk estimation we therefore hope to provide a few ideas and possible explanations to some of the puzzles concerning how we think about risk in international financial markets. 
Chapter 1: Introduction 


\section{CHAPTER 2 A DOWNSIDE RISK FRAMEWORK FOR OPTIMAL PORTFOLIO SELECTION ${ }^{1}$}

In this chapter we develop a portfolio selection model which allocates financial assets by maximising expected return subject to the constraint that the expected maximum loss should meet the Value-at-Risk limits set by an individual or institution. Similar to the mean-variance approach a performance index like the Sharpe index is constructed. Furthermore when expected returns are assumed to be normally distributed we show that the model provides almost identical results to the mean-variance approach ${ }^{2}$. We provide an empirical analysis using two risky assets: US stocks and bonds. The results highlight the influence of both non-normal characteristics of the expected return distribution and the length of investment time horizon on the optimal portfolio selection.

Modern portfolio theory aims to allocate assets by maximising the expected risk premium per unit of risk. In a mean-variance framework risk is defined in terms of the possible

This chapter is based on R. A. Campbell, R. Huisman and K. G. Koedijk, ëOptimal Portfolio Selection in a Value-at-Risk Framework,í forthcoming in the Journal of Banking and Finance (2001).

2 A slightly alternative specification on the downside risk constraint provides for the exact specification for normally distributed returns. 
variation of expected portfolio returns. The focus on standard deviation as the appropriate measure for risk implies that investors weigh the probability of negative returns equally against positive returns. However it is a stylised fact that the distribution of many financial return series are non-normal, with skewness and kurtosis pervasive ${ }^{3}$. Furthermore there is ample evidence that agents often treat losses and gains asymmetrically. There is a wealth of experimental evidence for loss aversion, see for example Kahneman, Knetsch and Thaler (1990). The choice therefore of mean-variance efficient portfolios is likely to give rise to an inefficient strategy for optimising expected returns for financial assets whilst minimising risk. It would therefore be more desirable to focus on a measure for risk that is able to incorporate any non-normality in the return distributions of financial assets. Indeed risk measures such as semi-variance were originally constructed in order to measure the negative tail of the distribution separately.

Recent research by Harvey and Siddique (2000), Bekaert et al. (1998) and Das and Uppal (1999) indeed advocate the need to incorporate non-normalities into the portfolio allocation decision. Alternative distributional assumptions are now widely used in the risk management literature, where there is greater consensus on the probability of extreme returns being non-normally distributed. Much of mainstream finance rests however on the assumption of normality, so that a move away from the assumption of normally distributed returns is not particularly favoured; one drawback often stated is the loss in the possibility of moving between discrete and continuous time frameworks.

3 See among others Fama and Roll (1968), Rogalski and Vinso (1978), Boothe and Glassman (1987), Taylor (1986), Jansen and de Vries (1991), and Huisman, Koedijk, Kool, and Palm (1998). 
Chapter 2: A downside risk framework for optimal portfolio selection

However it is precisely this simplifying approach, whereby any deviations from the square root of time rule are ignored, which needs to be incorporated into current finance theory. The ability to focus on additional moments in the return distribution with the possibility of allowing for skewed or leptokurtotic distributions enables additional risk factors (along with the use of standard deviation) to be included into the optimal portfolio selection problem.

We develop an optimal portfolio selection model that maximises expected return subject to a downside risk constraint rather than standard deviation alone. In our approach downside risk is written in terms of portfolio Value-at-Risk, so that additional risk resulting from any non-normality may be used to estimate the portfolio Value-at-Risk. This enables a much more generalised framework to be developed, with the distributional assumption most appropriate to the type of financial assets to be employed. We develop a performance index similar to the Sharpe ratio, and, for the case that financial assets are assumed to be normally distributed, provide a model similar to the mean-variance approach. The adoption of Value-at-Risk also provides us with the tools to incorporate much of the current research into risk management directly into the portfolio selection process. We are therefore able to provide a simple and powerful methodology for portfolio selection and management; whereby alternative distributional assumptions and hence risk factors may be incorporated into the optimal portfolio decision.

The outline of the chapter is as follows. We introduce the framework in the following section. The third section then provides empirical results of the optimal portfolio allocation for a US investor. We also shall address the importance of the non-normal characteristics 
Chapter 2: A downside risk framework for optimal portfolio selection of expected return distributions in such a framework. Conclusions and practical implications are drawn in the final section.

\subsection{PORTFOLIO SELECTION UNDER SHORTFALL CONSTRAINTS}

Portfolio selection under shortfall constraints has its origins in the work by Roy (1952) on safety-first. Roy defines the shortfall constraint such that the probability of the portfolio value falling below a specified disaster level is limited to a specified disaster probability. Although the concept of using shortfall constraints is more in line with investorsí perception to risk, their applicability was rather limited since the disaster levels, minimum returns, confidence levels or disaster probabilities were hard to specify. Further developments therefore, for example by Arzac and Bawa (1977), who provide a market equilibrium analysis, were largely ignored. The issue has however recently re-emerged since the enormous development in risk management techniques for estimating probabilities of disaster occurring. Leibowitz and Kogelman (1991), and Lucas and Klaassen (1998) for example construct portfolios by maximising expected return subject to a shortfall constraint, defined such that a minimum return should be gained over a given time horizon for a given confidence level.

We extend the literature on asset allocation subject to shortfall constraints. We address the criticism concerning the definition of disaster levels and probabilities through the use of Value-at-Risk (VaR), and develop a market equilibrium model for portfolio selection, which allows for alternative parametric distributions to be used. Banks and financial 


\section{Chapter 2: A downside risk framework for optimal portfolio selection}

institutions have adopted VaR as the measure for market risk ${ }^{4}$, whereby VaR is defined as the maximum expected loss on an investment over a specified horizon given some confidence level ${ }^{5}$. For example a $99 \%$ VaR for a 10-day holding period ${ }^{6}$, implies that the maximum loss incurred over the next 10 days should only exceed the VaR limit once in every 100 cases. It therefore reflects the potential downside risk faced on investments in terms of nominal losses. Introducing Value-at-Risk as a shortfall constraint into the portfolio selection decision, so that the portfolio manager or investor is highly concerned about the value of the portfolio falling below the VaR constraint, is much more in fitting with individual perception to risk and more in line with the constraints which management currently face. The advantage being that the shortfall constraint is then clearly defined in terms of a widely accepted market risk measure.

In the framework developed the measure for risk is defined in terms of the VaR over and above the risk free rate of return on the initial wealth. The portfolio is then selected to maximise expected return subject to the level of risk. The final choice of portfolio, including the borrowing and lending decision will therefore meet the specified VaR limit. $\mathrm{VaR}$ is therefore used as an ex-ante market risk control measure, extending the richness of VaR as a risk management tool. Basak and Shapiro (1999) also develop a model of optimal portfolio selection using a Value-at-Risk constraint in a continuous time framework. However the use of continuous time results in the premise that returns are normally distributed, so that any deviation from normality is ignored. Developing upon the

See Jorion (1997) for a comprehensive introduction into Value-at-Risk methodology.

In practice these confidence levels for Value-at-Risk range from $95 \%$ through $99 \%$, whereby the Basle Committee recommends $99 \%$. 
framework as laid out by Arzac and Bawa (1977), we provide a model in terms of downside risk, so that the optimal portfolio is determined in terms of its Value-at-Risk, and a performance index similar to the Sharpe ratio is developed. In this way we are able to leave the distributional assumptions about the structure of the tails of the distribution or any skewness to that most in accordance with the financial asset held. This has the advantage of allowing for non-normal payoffs as with most derivative products, providing a general but highly desirable model for optimal portfolio selection. We shall also see that under certain distributional assumptions the model collapses to the $\mathrm{CAPM}^{7}$, as developed by Sharpe (1964), Lintner (1965) and Mossin (1966). Since the model is able to encompass much of modern portfolio theory we are able to observe the effect on the portfolio decision induced by non-normalities.

\subsection{PORTFOLIO SELECTION MODEL}

In this section we present a portfolio construction model subject to a VaR limit set by the risk manager for a specified horizon. In other words we derive an optimal portfolio such that the maximum expected loss would not exceed the VaR for a chosen investment horizon at a given confidence level. Using $\mathrm{VaR}$ as the measure for risk in this framework is in accordance with the banking regulations in practice and provides a clear interpretation of investorsí behaviour of minimising downside risk. The degree of risk aversion is set

\footnotetext{
6 This is the VaR recommended by the Basle Committee for Banking Regulation used in establishing a bankís capital adequacy requirements.

7 See Arzac and Bawa (1977) for the derivation of CAPM.
} 
Chapter 2: A downside risk framework for optimal portfolio selection according to the VaR limit; hence avoiding the limitations of expected utility theory as to the degree of risk aversion which an investor is thought to exhibit.

\subsubsection{PORTFOLIO SELECTION PROBLEM AND DOWNSIDE RISK CONSTRAINT}

Suppose that we have an amount $\mathrm{W}(0)$ to be invested for an investment horizon $\mathrm{T}$, which we want to invest such that the portfolio meets a chosen VaR limit. This could be set for example by the risk management department, so that the financial institution meets the Basle capital adequacy requirement, or by the private investor according to his individual aversion to risk. This amount can therefore be invested along with an amount B representing borrowing $(B>0)$ or lending $(B<0)$. We assume $r_{f}$ is the interest rate at which the investor can borrow and lend for the period T. There are $\mathrm{n}$ available assets, and $\gamma(\mathrm{i})$ denotes the fraction invested in the risky asset, i. The $\gamma(\mathrm{i}) \mathrm{s}$ must therefore sum to one. Letting $\mathrm{P}(\mathrm{i}, \mathrm{t})$ be the price of asset $\mathrm{i}$ at time $\mathrm{t}$ (the current decision period is therefore when $\mathrm{t}=0$ ). The initial value of the portfolio, in equation (2.1) below, represents the budget constraint:

$$
W(0)+B=\sum_{i=1}^{n} \gamma(i) P(i, 0)
$$

The manager or investor therefore needs to choose the fractions $\gamma(\mathrm{i})$ to be invested with the initial wealth $\mathrm{W}(0)$ and the amount borrowed or lent at time 0 . Allocating the assets in the portfolio and choosing the amount to borrow or lend such that the maximum expected level of final wealth is achieved results in the definition of the portfolio allocation problem. 
Choosing the desired level of Value-at-Risk as $\mathrm{VaR}^{*}$ we therefore formulate the downside risk constraint as follows:

$$
\operatorname{Pr}\left\{W(0)-W(T, p) \geq V a R^{*}\right\} \leq(1-c)
$$

Pr denotes the expected probability conditioned on the information available at time zero for portfolio p. Equation (2.2) is equivalent to:

$$
\operatorname{Pr}\left\{W(T, p) \leq W(0)-V a \mathrm{R}^{*}\right\} \leq(1-c)
$$

Since VaR is the worst expected loss over the investment horizon $\mathrm{T}$ which can be expected with confidence level c, the investorís level of risk aversion is reflected in both the level of the VaR and the confidence level associated with it. The optimal portfolio, which is derived such that equation (2.3) holds, will therefore reflect this.

\subsubsection{OPTIMAL PORTFOLIO CONSTRUCTION}

The introduction of VaR however provides us with a shortfall constraint (denoted by equation (2.3)) that fits perfectly into the Arzac and Bawa framework. We therefore build upon their results to derive an optimal portfolio selection model. The investor is interested in maximising wealth at the end of the investment horizon. Let $r(p)$ be the expected total return on a portfolio $\mathrm{p}$ in period $\mathrm{T}$; assume that asset $\mathrm{i}$ is included with fraction $\gamma(\mathrm{i}, \mathrm{p})$ in portfolio $\mathrm{p}$. The expected wealth from investing in portfolio $\mathrm{p}$ at the end of the investment horizon becomes: 
Chapter 2: A downside risk framework for optimal portfolio selection

$$
E_{0}(W(T, p))=(W(0)+B)(1+r(p))-B\left(1+r_{f}\right)
$$

Substituting in for B as given in equation (2.1), we are able to express final wealth in terms of the risk-free rate of return and the expected portfolio risk premium $\left(r(p)-r_{f}\right)$ :

$$
E_{0}(W(T, p))=W(0)\left(1+r_{f}\right)+\left(\sum_{i=1}^{n} \gamma(i, p) P(i, 0)\right)\left(r(p)-r_{f}\right)
$$

Equation (2.5) shows that as long as the expected risk premium is positive, a risk-averse investor will always invest some fraction of his wealth in the risky assets. In order to determine the optimal portfolio that maximises the expected final wealth subject to the VaR constraint (2.3) we substitute (2.5) into the downside risk constraint, equation (2.3), and rearranging gives:

$$
\operatorname{Pr}\left\{r(p) \leq r_{f}-\frac{V a \mathrm{R}^{*}+W(0) r_{f}}{\sum_{i=1}^{n} \gamma(i, p) P(i, 0)}\right\} \leq(1-c)
$$

Since equation (2.6) simply defines the quantile $q(\mathrm{c}, \mathrm{p})$ that corresponds to probability $(1-\mathrm{c})$ of occurrence that can be read off the cdf of the expected return distribution for portfolio $\mathrm{p}$, we can use this definition of the quantile to derive the following expression from (2.6):

$$
\sum_{i=1}^{n} \gamma(i, p) P(i, 0)=\frac{V a \mathrm{R}^{*}+W(0) r_{f}}{r_{f}-q(c, p)}
$$


Substituting (2.6) back into (2.5) leads us to the following expression for the expected final wealth in terms of the quantile $q(c, p)$, which of course is negative:

$$
E_{0}(W(T, p))=W(0)\left(1+r_{f}\right)+\frac{\left(r(p)-r_{f}\right)}{\left(r_{f}-q(c, p)\right)}\left(V a \mathrm{R}^{*}+W(0) r_{f}\right)
$$

Dividing (2.8) by initial wealth $\mathrm{W}(0)$ we obtain the following expression for the expected return on the initial wealth:

$$
E_{0}\left(\frac{W(T, p)}{W(0)}\right)=\left(1+r_{f}\right)+\frac{\left(r(p)-r_{f}\right)}{\left(W(0) r_{f}-W(0) q(c, p)\right)}\left(V a \mathrm{R} *+W(0) r_{f}\right)
$$

It can be seen from equation (2.9) that the final expected return on wealth is maximised for an investor concerned about the downside risk by the portfolio maximising $S(p)$ in equation (2.10). We denote this maximising portfolio as $\mathrm{p}^{\prime}$, where $\mathrm{q}(\mathrm{c}, \mathrm{p})$ simply defines the quantile that corresponds to probability (1-c) of occurrence, which can be read off the cdf of the expected return distribution for the portfolio $\mathrm{p}$.

$$
p^{\prime}: \max _{p} S(p)=\frac{r(p)-r_{f}}{W(0) r_{f}-W(0) q(c, p)}
$$

Note that although initial wealth is in the denominator of $S(p)$ it does not affect the choice of the optimal portfolio since it is only a scale constant in the maximisation. The asset allocation process is thus independent of wealth. The advantage however of having initial wealth in the denominator is in its interpretation. $\mathrm{S}(\mathrm{p})$ equals the ratio of the expected risk premium offered on portfolio $\mathrm{p}$ to the risk, reflected by the maximum expected loss on 
Chapter 2: A downside risk framework for optimal portfolio selection

portfolio $\mathrm{p}$ that is incurred with probability 1 -c relative to the risk-free rate. Since the negative quantile of the return distribution multiplied by the initial wealth is the Value-atRisk associated with the portfolio for a chosen confidence level, we are able to derive an expression for the risk faced by the investor as $\varphi$. Letting $\operatorname{VaR}(\mathrm{c}, \mathrm{p})$ denote portfolio pís Value-at-Risk, the denominator of (2.10) may be written as:

$$
\varphi(c, p)=W(0) r_{f}-V a R(c, p)
$$

Such a measure for risk is in fitting with investorsí behaviour of focussing on the risk free rate of return as the benchmark return with risk being measured as the potential for losses to be made with respect to the risk free rate as the point of reference. Indeed the measure for risk can be seen as a possible measure for regret, since it measures the potential opportunity loss of investing in risky assets. Investors will therefore only accept greater returns if they can tolerate the regret occurring from the greater potential wealth-at-risk. The risk-return ratio $S(p)$, which is maximised for the optimal portfolio $p^{\prime}$ can therefore be written as:

$$
p^{\prime}: \max _{p} S(p)=\frac{r(p)-r_{f}}{\varphi(c, p)}
$$

$\mathrm{S}(\mathrm{p})$ is thus a performance measure like the Sharpe index that can be used to evaluate the efficiency of portfolios (see Sharpe 1994 for more details). Indeed under the assumption that expected portfolio returns are normally distributed and the risk free rate is zero, $\mathrm{S}(\mathrm{p})$ collapses to a multiple of the Sharpe index. In this case the VaR is expressed as a multiple of the standard deviation of the expected returns so that the point at which both 
performance indices are maximised will lead to the same optimal portfolio being chosen. Only a minimal difference in the optimal portfolio weights occurs for positive risk free rates for a small time horizon and so both approaches will lead to almost identical results, a result that we shall come back to in our empirical analysis. Indeed reformulating the downside risk constraint in equation (2.2) to include the loss made from borrowing the amount $\mathrm{B}$ at the risk free rate results in the risk measure in equation (2.11) simplifying to the portfolio $\mathrm{VaR}$ and hence provides identical results to the Sharpe ratio for normally distributed returns ${ }^{8}$.

We prefer the original specification where the risk measure includes the concept that the investor is concerned with loss compared to a benchmark, and therefore continue with this framework for the empirical results. Since our performance index $\mathrm{S}(\mathrm{p})$ does not rely on any distributional assumptions it has the advantage of being able to incorporate non-normalities into the asset allocation problem through the use of other distributional assumptions. The existence of non-normalities may lead to the choice of different optimal portfolios, an empirical investigation of which we shall encounter later.

The optimal portfolio that maximises $\mathrm{S}(\mathrm{p})$ in $(2.12)$ is chosen independently from the level of initial wealth. It is also independent from the desired VaR, since the risk measure $\varphi$ for the various portfolios depends on the estimated portfolio VaR rather than the desired Value-at-Risk. Investors first allocate the risky assets and then, the amount of borrowing or

8 Including the known loss from the amount of borrowing the downside risk constraint may be reformulated as follows, $\operatorname{Pr}\left\{W(0)-W(T, p) \geq V a \mathrm{R} *-r_{f} B\right\} \leq(1-c)$. This leads to a performance index containing only the quantile, or $\operatorname{VaR}(\mathrm{c}, \mathrm{p})$ in the denominator. 
Chapter 2: A downside risk framework for optimal portfolio selection

lending will reflect by how much the VaR of the portfolio differs from the VaR limit set; thus two-fund separation holds like in the mean-variance framework. However since the investorsí degree of risk aversion is captured by the chosen Value-at-Risk level, the amount of borrowing or lending required to meet the VaR constraint may be determined. This is a significant benefit of the model, and is practical advantageous, with investors easily and accurately being able to determine the desired risk-return trade off with the necessary correct amount of borrowing or lending. The amount to be borrowed is found by substituting (2.7) into equation (2.1), multiplying through by $\mathrm{W}(0)$ and substituting in for equation (2.11). The amount to be borrowed is denoted by equation (2.13):

$$
B=\frac{W(0)\left(V a \mathrm{R}^{*}-V a \mathrm{R}\left(c, p^{\prime}\right)\right)}{\varphi^{\prime}\left(c, p^{\prime}\right)}
$$

The optimal portfolio is independent of the distributional assumption, so that the model has been derived solely on the premise that investors wish to maximise expected return subject to a downside risk constraint.

\subsection{OPTIMAL PORTFOLIO SELECTION FOR US STOCKS AND BONDS}

In order to determine the effect that deviations from normality, and the time horizon chosen for the VaR level we have estimated the optimal portfolios for a US investor using US Stocks and Bonds such that our downside risk constraint over various time horizons is met. We use data obtained from Datastream for the S\&P 500 Composite Return Index for the US, the 10- Year Datastream Benchmark US Government Bond Return Index and the 3- Month US Treasury Bill rate for the risk free rate. 


\section{TABLE 2 .1 SUMMARY STATISTICS}

The table gives the summary statistics for the S\&P 500 Composite Returns Index and the 10-Year Datastream US Benchmark Government Bond Index over the period January 1990 - December 1998.

S\&P 500 COMPOSITE RETURN INDEX

\begin{tabular}{lrrr} 
& Daily & Bi-Weekly & \multicolumn{1}{c}{ Monthly } \\
\hline Observations & 2364 & 248 & 132 \\
Average Return & 0.000528 & 0.00523 & 0.010804 \\
Standard Deviation & 0.007717 & 0.028459 & 0.037896 \\
Maximum Return & 0.058101 & 0.098783 & 0.106718 \\
Minimum Return & -0.03532 & -0.07666 & -0.11075 \\
Skewness & 0.179661 & 0.170371 & -0.06104 \\
Kurtosis & 6.78397 & 3.73567 & 3.45994 \\
\hline
\end{tabular}

10 YeAR DATASTREAM

US BENCHMARK GOVERNMENT BOND INDEX

\begin{tabular}{lrrr} 
& Daily & Bi-Weekly & Monthly \\
\hline Observations & 2364 & 248 & 132 \\
Average Return & 0.000331 & 0.003317 & 0.006572 \\
Standard Deviation & 0.003972 & 0.012421 & 0.018705 \\
Maximum Return & 0.016462 & 0.031485 & 0.039118 \\
Minimum Return & -0.02826 & -0.04402 & -0.05199 \\
Skewness & -0.39087 & -0.38734 & -0.46151 \\
Kurtosis & 6.23627 & 3.34004 & 2.8721 \\
\hline
\end{tabular}


Chapter 2: A downside risk framework for optimal portfolio selection

We employ daily data from these US indices from January 1990 until December 1998, providing us with 2364 observations. The average annual return on the S\&P 500 over the sample period was $16.81 \%$, just over twice as high as the average annual return on the 10 year Government Bond Index of $8.35 \%$. The annual standard deviation is also higher on the S\&P 500 at $13.42 \%$ per annum, compared to the less volatile nature of the Government Bonds with an annual standard deviation of only $6.31 \%$.

Looking at the alternative frequencies in Table 2.1, we see that the monthly average return is naturally greater than the daily return; however the standard deviation of the distribution is also greater, and is even greater than the square root of time rule would suggest. This provides an indication of autocorrelation. We also see that for all three data frequencies significant skewness and kurtosis is prevalent.

\subsubsection{EMPIRICAL DISTRIBUTION}

To find the portfolio which maximises the performance index $S(p)$ in (2.12) we estimate both the expected return $\mathrm{r}(\mathrm{p})$ and the Value-at-Risk for various combinations of US stocks and bonds, using the daily, biweekly and monthly data over the sample period. Plotting the risk return trade off provides us with an efficient VaR frontier for a given confidence level for $\mathrm{VaR}$, moving from a portfolio containing $100 \%$ bonds to a $100 \%$ investment into stocks. In Figure 2.1 we have plotted efficient $\mathrm{VaR}$ frontiers using daily data, whereby alternative distributional assumptions have been used to estimate $\varphi$, the parameter for risk. 


\section{FIGURE 2.1 EFFICIENT VAR FRONTIER ñ DAILY DATA AND DAILY VAR AT}

\section{5\% CONFIDENCE LEVEL}

The figure presents the risk return trade off for portfolios of Stocks and Bonds whereby risk is measured by the downside risk measure $\varphi$ of the portfolio at the 95\% confidence level. The returns and VaR estimates are obtained using daily data on the S\&P 500 Composite Returns Index and the 10- Year Datastream US Benchmark Government Bond Index for the period January 1990 until December 1998. We present the efficient frontier for the empirical distribution, the parametric normal approach and under the assumption of a Student-t distribution with 5 degrees of freedom.

\section{Efficient VaR Frontier: 95\% Confidence Daily Data}

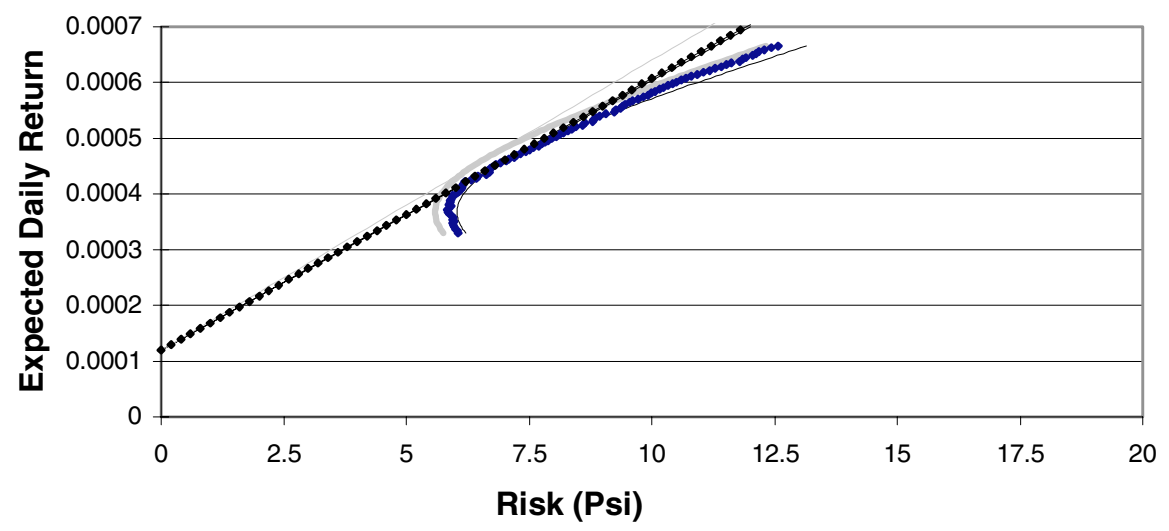

\begin{tabular}{|ll|}
\hline $\begin{array}{l}\text { Historical } \\
- \text { - CML Historical }\end{array}$ & Normal \\
\cline { 2 - 2 }
\end{tabular}


Chapter 2: A downside risk framework for optimal portfolio selection

The efficient $\mathrm{VaR}$ frontier is similar to a mean-variance frontier except for the definition of risk: VaR relative to the benchmark return $(\varphi)$ instead of standard deviation $(\sigma)$. The empirical distribution provides the true risk-return trade off using this risk measure, as observed in financial markets; however the greater the time horizon for the investment then the less precise the efficient $\mathrm{VaR}$ frontier. In order to determine the exact proportion of the portfolio which needs to be held in cash, we need to know the investorís risk profile. The level set for VaR, which includes the choice for the confidence level associated with the VaR level, determines this. In the empirical example below we have set the desired VaR level as the $95 \%$ VaR from the historical distribution. This provides us with a benchmark with which we can compare the alternative distributional assumptions and various time horizons used for the investment period.

An investor who wants to be $95 \%$ confident that his or her wealth will not drop by more than the daily VaR limit whilst still attaining the highest possible return therefore selects the point on the efficient VaR frontier where return per unit of risk is maximised. To determine the optimal allocation between stocks and bonds, we set the risk free rate at $4.47 \%$, the last available 3-month Treasury bill rate in the sample period. For an investor with a VaR limit at the $95 \%$ confidence level the optimal allocation between US stocks and bonds occurs when $36 \%$ of wealth is held in stocks and $64 \%$ in bonds. The combinations for stocks and bonds for a variety of confidence levels are provided in the first two columns of Table 2.2, and the portfolio VaR is given in the third column. 


\section{TABLE 2.2 OPTIMAL PORTFOLIOS USING EMPIRICAL DISTRIBUTION FOR}

\section{VAR ESTIMATION}

Data on the S\&P 500 Composite Returns Index and the 10- Year Datastream US Benchmark Government Bond Index over the period January 1990 - December 1998 are used to find the optimal portfolios. Optimal portfolios consisting of US Stocks and Bonds are found at the point at which the risk-return trade off (equation (2.10)) is maximised. The risk free return is the rate on the last periodsi one month Treasury bill, (4.47\%). The VaRs for $\$ 1000$ held in the portfolios are given for a daily time horizon, where the historical distribution is used to estimate the Value-at-Risk.

\section{Daily}

\begin{tabular}{cccc} 
Confidence Level & Stocks & Bonds & Portfolio VaR $(\$)$ \\
\hline $\mathbf{9 5} \%$ & $36 \%$ & $64 \%$ & -6.84 \\
$\mathbf{9 6} \%$ & $40 \%$ & $60 \%$ & -7.66 \\
$\mathbf{9 7 \%}$ & $33 \%$ & $67 \%$ & -7.90 \\
$\mathbf{9 8} \%$ & $45 \%$ & $55 \%$ & -10.22 \\
$\mathbf{9 9} \%$ & $34 \%$ & $66 \%$ & -11.40
\end{tabular}

Naturally the greater the confidence level chosen in association with the Value-at-Risk then the greater the portfolio Value-at-Risk. Absolute portfolio VaR is given in the final column. In order to ensure that the portfolio meets the desired VaR, in accordance with equation (2.13) a greater proportion of the portfolio will be needed to be held at the risk free rate the higher the confidence level associated with the VaR level set: a movement along the Capital Market Line, also shown in Figure 2.1. The final proportions of the portfolio optimising the risk return trade off for the chosen VaR level are provided in Table 2.3 . 
Chapter 2: A downside risk framework for optimal portfolio selection

\section{TABLE 2.3 OPTIMAL PORTFOLIOS TO MEET VAR CONSTRAINT UNDER}

\section{EMPIRICAL DISTRIBUTION}

Under the assumption that expected returns are distributed as in the past the optimal portfolio allocation is found such that the decision to borrow or lend is incorporated. The final optimal portfolios are found where various VaR constraints are met. These have been arbitrarily chosen to exemplify variations in individuals risk-return profiles. The data used are as described in Table 2.2, for $\$ 1000$ held in the portfolio, whereby the historical distribution at the 95\% empirical level is used to estimate the Value-at-Risk.

\section{Daily}

\begin{tabular}{cccc} 
Confidence Level & Stocks & Bonds & Cash \\
\hline $\mathbf{9 5 \%}$ & $36.00 \%$ & $64.00 \%$ & $0.00 \%$ \\
$\mathbf{9 6 \%}$ & $35.78 \%$ & $53.68 \%$ & $10.54 \%$ \\
$\mathbf{9 7 \%}$ & $28.63 \%$ & $58.12 \%$ & $13.25 \%$ \\
$\mathbf{9 8 \%}$ & $30.30 \%$ & $37.04 \%$ & $32.66 \%$ \\
$\mathbf{9 9 \%}$ & $20.55 \%$ & $39.89 \%$ & $39.56 \%$
\end{tabular}

Bi-Weekly

\begin{tabular}{cccc} 
Confidence Level & Stocks & Bonds & Cash \\
\hline $\mathbf{9 5 \%}$ & $39.00 \%$ & $61.00 \%$ & $0.00 \%$ \\
$\mathbf{9 6 \%}$ & $23.38 \%$ & $57.24 \%$ & $19.38 \%$ \\
$\mathbf{9 7 \%}$ & $31.66 \%$ & $28.08 \%$ & $40.26 \%$ \\
$\mathbf{9 8 \%}$ & $30.33 \%$ & $21.96 \%$ & $47.70 \%$ \\
$\mathbf{9 9 \%}$ & $23.20 \%$ & $25.14 \%$ & $51.66 \%$
\end{tabular}

\section{Monthly}

\begin{tabular}{cccc} 
Confidence Level & Stocks & Bonds & Cash \\
\hline $\mathbf{9 5 \%}$ & $90.00 \%$ & $10.00 \%$ & $0.00 \%$ \\
$\mathbf{9 6 \%}$ & $80.04 \%$ & $6.02 \%$ & $13.94 \%$ \\
$\mathbf{9 7 \%}$ & $62.77 \%$ & $11.08 \%$ & $26.15 \%$ \\
$\mathbf{9 8 \%}$ & $48.62 \%$ & $0.49 \%$ & $50.89 \%$ \\
$\mathbf{9 9 \%}$ & $40.83 \%$ & $2.15 \%$ & $57.02 \%$
\end{tabular}

We can see how sensitive the portfolio selection decision is to changes in the confidence level associated with the Value-at-Risk limit. Allocating 36\% in Stocks and $64 \%$ in Bonds generates a $95 \%$ VaR on the portfolio of $\$-6.86$ and of course since this is the desired VaR 
no borrowing or lending is required to meet the $\mathrm{VaR}$ constraint. If however the risk manager desires greater confidence in the probability that the initial wealth will not drop by more than the VaR level, then the VaR associated with the portfolio allocation will be greater than the VaR limit and hence results in too much risk being taken ${ }^{9}$. In order to meet the benchmark VaR less risk will have to be taken and hence a proportion of the initial wealth is lent at the risk free rate. This is provided in the final column where we see that the greater the confidence level, hence the lower the risk tolerance of the investor, the greater the proportion of wealth that needs to be lent at the risk free rate ${ }^{10}$.

The use of the empirical distribution results in the stock proportions not being a monotonic function of the confidence level. If however we assume that the future distribution of returns can be accurately proxied by the normal distribution, the only risk factor in our downside risk measure is the standard deviation of the distribution. This means that the quantile estimate is merely a multiple of standard deviations, and for short time horizons our risk measure $\varphi$ in equation (2.11) depends almost entirely on the multiple of the standard deviation. This results in the risk-return trade off being almost identical to that derived under the mean-variance framework where the Sharpe ratio is maximised. Of course since we also have the possibility of assuming different distributional assumptions, we need not constrain ourselves to optimising our portfolio according to the first two moments of the distribution only and hence are able to include the possibilities of nonnormalities into asset allocation. We therefore compare the optimal allocation of assets

9 The choice of a higher confidence level will by definition result in a higher Value-at-Risk. 
Chapter 2: A downside risk framework for optimal portfolio selection

derived using both the normal distribution and a fatter tailed distribution, the student-t, whereby we use the same sample period of data as before.

\subsubsection{ALTERNATIVE PARAMETRIC DISTRIBUTIONS}

From Figure 2.1 we saw that at the $95 \%$ VaR level the assumption of normality reflects the actual risk-return trade off fairly well. On average the assumption of normality for the future distribution of returns at the $95 \%$ level means that the risk is only slightly overestimated for a given level of return. The risk is minimised at the optimal allocation of $40 \%$ stocks and $60 \%$ bonds for daily VaR. Regardless of the confidence level, chosen for the VaR, we see that the optimal combination of risky assets is the same. Since the VaR is a multiple of the portfolio standard deviation then the assumption of normality renders the investors attitude to risk unimportant in the optimisation process. The use of longer frequency data yields an optimum of $45 \%$ stocks and $55 \%$ bonds for bi-weekly data, and $61 \%$ stocks and $39 \%$ bonds for monthly data. The maximisation also occurs at the same point as when maximising the Sharpe ratio for all types of frequencies used ${ }^{11}$. However through the use of $\mathrm{VaR}$ we are able to provide greater insight into the actual risk return trade off facing the investor, without having to resort to the use of specifying an individualís utility function for consumption. The exact portfolio proportions in stocks,

10 In a similar manner specifying a confidence level below that used for the optimisation the risk manager would want to take on additional risk by borrowing additional funds at the risk free rate, and going short in the 3-month Treasury Bill.

11 Indeed for daily, bi-weekly and monthly time horizons the difference is negligible, resulting in the same optimum being found. The greater the investment time horizon however, the greater the risk free rate of return, and hence the two risk measures will provide a different optimal points. 
Chapter 2: A downside risk framework for optimal portfolio selection bonds and cash to meet the $95 \%$ empirical VaR are given in Table 2.4 for various confidence levels and again for the various time horizons.

\section{TABLE 2.4 OPTIMAL PORTFOLIOS TO MEET VAR CONSTRAINT UNDER}

\section{NORMALITY}

Under the assumption that expected returns are normally distributed the optimal portfolio allocation is found such that the decision to borrow or lend is incorporated. The final optimal portfolios are found where various VaR constraints are met. These have been arbitrarily chosen to exemplify variations in individualis risk-return profiles. The data used are as described in Table 2.2, for $\$ 1000$ held in the portfolio, whereby the historical distribution at the 95\% empirical level is used to estimate the Value-at-Risk.

\section{Daily}

\begin{tabular}{cccc} 
Confidence Level & Stocks & Bonds & Cash \\
\hline $\mathbf{9 5 \%}$ & $38.22 \%$ & $57.33 \%$ & $4.44 \%$ \\
$\mathbf{9 6 \%}$ & $35.81 \%$ & $53.72 \%$ & $10.46 \%$ \\
$\mathbf{9 7 \%}$ & $33.24 \%$ & $49.86 \%$ & $16.90 \%$ \\
$\mathbf{9 8 \%}$ & $30.34 \%$ & $45.51 \%$ & $24.15 \%$ \\
$\mathbf{9 9 \%}$ & $26.67 \%$ & $40.01 \%$ & $33.32 \%$
\end{tabular}

Bi-Weekly

\begin{tabular}{cccc} 
Confidence Level & Stocks & Bonds & Cash \\
\hline $\mathbf{9 5 \%}$ & $48.47 \%$ & $59.24 \%$ & $-7.71 \%$ \\
$\mathbf{9 6 \%}$ & $41.73 \%$ & $51.01 \%$ & $7.26 \%$ \\
$\mathbf{9 7 \%}$ & $35.64 \%$ & $43.56 \%$ & $20.80 \%$ \\
$\mathbf{9 8 \%}$ & $29.85 \%$ & $36.48 \%$ & $33.66 \%$ \\
$\mathbf{9 9 \%}$ & $23.76 \%$ & $29.05 \%$ & $47.19 \%$
\end{tabular}

\section{Monthly}

\begin{tabular}{cccc} 
Confidence Level & Stocks & Bonds & Cash \\
\hline $\mathbf{9 5 \%}$ & $84.33 \%$ & $53.91 \%$ & $-38.24 \%$ \\
$\mathbf{9 6 \%}$ & $49.54 \%$ & $31.68 \%$ & $18.78 \%$ \\
$\mathbf{9 7 \%}$ & $32.88 \%$ & $21.02 \%$ & $46.10 \%$ \\
$\mathbf{9 8 \%}$ & $22.72 \%$ & $14.52 \%$ & $62.76 \%$ \\
$\mathbf{9 9 \%}$ & $15.28 \%$ & $9.77 \%$ & $74.96 \%$
\end{tabular}




\section{Chapter 2: A downside risk framework for optimal portfolio selection}

The non-parametric nature of the empirical distribution however, led to the changing optimum allocation of assets for various confidence levels, whereby the optimal portfolio selection resulted in a proportionally greater increase in lending to meet the desired VaR level for higher confidence levels. Under the assumption of normality with standard deviation crucial in the measure for risk this effect is not captured. Unfortunately the assumption of normality underestimates the risk return trade off as presented in the efficient VaR frontiers. This can be seen graphically in Figure 2.2 where the efficient VaR frontiers at the $99 \%$ confidence level can be compared for a daily time horizon using both the normal and the empirical distributions. It appears that for a desired confidence level of $99 \%$, for all time horizons for $\mathrm{VaR}$, too aggressive an investment strategy results.

The level of risk, as measured by the empirical VaR for the portfolio, is higher for all combinations of stocks and bonds than captured by the use of standard deviation alone. The greater the deviation from normality ${ }^{12}$ the greater the underestimation of risk as we move to higher confidence levels for the VaR. The greater probability of extreme negative returns in the empirical distribution implies greater downside risk than is captured by the measure of standard deviation alone. The use therefore of the normal distribution to assess the risk-return trade off will result in an incorrect allocation of assets for investors with low risk tolerance and risk managers wishing to set $99 \%$ confidence levels. The nature of the student-t distribution with its thin waist and fat tails gives rise to a smaller estimation of the portfolio VaR for lower confidence levels, and to a greater estimation for higher confidence levels.

12 See for example Huisman, Koedijk and Pownall (1998). 


\section{FIGURE 2.2 EFFICIENT VAR FRONTIER ñ DAILY DATA AND DAILY VAR AT}

\section{9\% CONFIDENCE LEVEL}

The figure presents the risk return trade off for portfolios of Stocks and Bonds whereby risk is measured by the downside risk measure $\varphi$ of the portfolio at the 99\% confidence level. The returns and VaR estimates are obtained using daily data on the S\&P 500 Composite Returns Index and the 10- Year Datastream US Benchmark Government Bond Index for the period January 1990 until December 1998. We present the efficient frontier for the empirical distribution, the parametric normal approach and under the assumption of a Student-t distribution with 5 degrees of freedom.

\section{Efficient VaR Frontier: 99\% Confidence Daily Data}

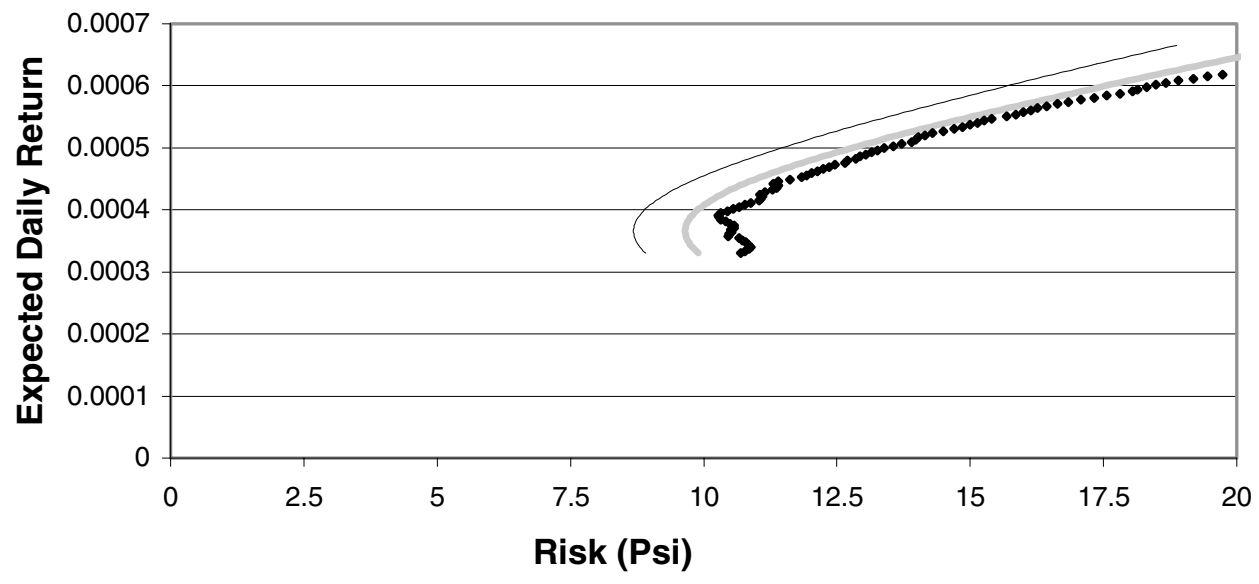

- Historical - Normal - Student-t(5) 
From Figure 2.2 we see that for daily VaR it is indeed the case that at the $99 \%$ confidence level the use of normality to estimate VaR results in too high an allocation into stocks. It would therefore appear to be more appropriate to use the student-t distribution with 5 degrees of freedom ${ }^{13}$. The affect however is not so severe when a bi-weekly or a monthly time horizon is used.

As we move to higher confidence levels, for a shorter time horizon for the VaR estimation we find that it becomes more important to incorporate the additional downside risk from fat tails into the risk-return trade off. The proportions held in the risky assets are the same as under the assumption of normality, however the portfolio risk is greater. To ensure that the final portfolio selection meets the same desired VaR level a greater proportion of the portfolio needs to be held at the risk free rate. This is presented in Table 2.5.

13 The smaller the number of degrees of freedom used to parameterise the student-t distribution the fatter the tails of the distribution and the greater the severity of the difference between the normal distribution. Tail index estimation techniques may be adopted for the correct estimation of the degrees of freedom for the student-t distribution, see Huisman et al. (2000) for a robust estimator in small samples. Adopting this approach we find the use of five degrees of freedom throughout the empirical analysis provides consistent results. 


\section{TABLE 2.5 OPTIMAL PORTFOLIOS TO MEET VAR CONSTRAINT UNDER}

\section{STUDENT-T}

Under the assumption that expected returns are student-t distributed, with 5 degrees of freedom, the optimal portfolio allocation is found such that the decision to borrow or lend is incorporated. The final optimal portfolios are found where various VaR constraints are met. These have been arbitrarily chosen to exemplify variations in individuals risk-return profiles. The data used are as described in Table 2.2, for $\$ 1000$ held in the portfolio, whereby the historical distribution at the 95\% empirical level is used to estimate the Value-at-Risk.

\begin{tabular}{cccc}
$\begin{array}{l}\text { Daily } \\
\text { Confidence Level }\end{array}$ & Stocks & Bonds & Cash \\
\hline $\mathbf{9 5 \%}$ & $40.38 \%$ & $60.57 \%$ & $-0.95 \%$ \\
$\mathbf{9 6 \%}$ & $36.99 \%$ & $55.49 \%$ & $7.51 \%$ \\
$\mathbf{9 7 \%}$ & $33.33 \%$ & $50.00 \%$ & $16.67 \%$ \\
$\mathbf{9 8 \%}$ & $29.14 \%$ & $43.72 \%$ & $27.14 \%$ \\
$\mathbf{9 9 \%}$ & $23.73 \%$ & $35.59 \%$ & $40.68 \%$
\end{tabular}

\section{Bi-Weekly}

\begin{tabular}{cccc} 
Confidence Level & Stocks & Bonds & Cash \\
\hline $\mathbf{9 5 \%}$ & $55.59 \%$ & $67.94 \%$ & $-23.54 \%$ \\
$\mathbf{9 6 \%}$ & $44.89 \%$ & $54.87 \%$ & $0.24 \%$ \\
$\mathbf{9 7 \%}$ & $35.84 \%$ & $43.81 \%$ & $20.35 \%$ \\
$\mathbf{9 8 \%}$ & $27.73 \%$ & $33.89 \%$ & $38.38 \%$ \\
$\mathbf{9 9 \%}$ & $19.65 \%$ & $24.02 \%$ & $56.34 \%$
\end{tabular}

Monthly

\begin{tabular}{cccc} 
Confidence Level & Stocks & Bonds & Cash \\
\hline $\mathbf{9 5 \%}$ & $190.43 \%$ & $121.75 \%$ & $-212.17 \%$ \\
$\mathbf{9 6 \%}$ & $62.62 \%$ & $40.04 \%$ & $-2.66 \%$ \\
$\mathbf{9 7 \%}$ & $33.31 \%$ & $21.30 \%$ & $45.39 \%$ \\
$\mathbf{9 8 \%}$ & $19.83 \%$ & $12.68 \%$ & $67.49 \%$ \\
$\mathbf{9 9 \%}$ & $11.43 \%$ & $7.31 \%$ & $81.26 \%$
\end{tabular}




\subsection{CONCLUDING REMARKS}

Focussing on downside risk as an alternative measure for risk in financial markets has enabled us to develop a framework for portfolio selection that moves away from the standard mean-variance approach. The measure for risk depends on a portfolioís potential loss function, itself a function of portfolio Value-at-Risk. Introducing Value-at-Risk into the measure for risk has the benefit of allowing the risk return trade off to be analysed for various associated confidence levels. Since the riskiness of an asset increases with the choice of the confidence level associated with the downside risk measure, risk becomes a function of the individuals risk aversion level. This enables us to analyse financial riskreturn trade-offs as observed in financial markets with an extra dimension: the confidence level associated with the riskiness of the financial asset. The portfolio selection problem is still to maximise expected return, however whilst minimising the downside risk as captured by Value-at-Risk. This allows us to develop a very generalised framework for portfolio selection. Alternative estimation procedures for Value-at-Risk therefore provide alternative optimal portfolios. This has indeed been shown for US stocks and Bonds where the time horizon also plays a crucial role in which distributional assumption should be used. Indeed the use of certain parametric distributions such as the normal or the student-t allows for a market equilibrium model to be derived. Furthermore the assumption of normality enables the model to collapse to the CAPM, which enables us to test the VaR approach to portfolio selection when other distributional assumptions are assumed. The ability of the framework to nest much of the mainstream finance literature enables the results to be compared easily to the current literature. We can see for example just how 
Chapter 2: A downside risk framework for optimal portfolio selection great the impact is on the portfolio selection decision from non-normalities, alternative time horizons, and alternative risk specifications. This leads us to be able to establish a new perspective with which the risk return trade off is measured, a result that shall be taken up further in the following chapters. 


\section{CHAPTER 3 FAT TAILS IN FINANCIAL RISK MANAGEMENT $^{14}$}

To ensure a competent regulatory framework with respect to Value-at-Risk for establishing Bankís capital adequacy requirements, as promoted by the Basle Committee on banking supervision, then the parametrical approach to estimate VaR needs to incorporate fat tails, apparent in the return distributions of financial assets. This chapter provides a simple method to obtain accurate parametric VaR measures, by including a specific measure for the tail fatness of an assetis return distribution: VaR-x. We provide evidence for the accuracy of these VaR-x estimates by comparing different parametric VaR estimators for bi-weekly returns on US stock and bond returns.

The quest for reliable Risk Management techniques has grown in response to higher volatility and instability on global financial markets, compounded by the enormous growth in trading activity and international exposure. One need only think of the losses made from recent currency and stock market crashes as well as those resulting from the perilous

14 This chapter is based on R. Huisman, K. G. Koedijk and R. A. Pownall, 'Fat Tails in Financial Risk Management' Journal of Risk (1998). 
positions taken for example by Barings, Daiwa, Orange County, and Metallgesellschaft. Value-at-Risk (VaR) is one such risk management technique developed to improve the management of downside risk. It aims to summarise risk, by estimating the worst expected loss over a chosen time horizon within a given confidence interval. The methodology behind Value-at-Risk is thus based on the probabilities associated with large negative returns and hence highlights how financial institutions have had to become more concerned with managing this downside risk. Only through the use of such risk management methods can the exposure towards large negative movements in financial markets be controlled and reduced. However their benefit rests primarily on the accuracy of the Value-at-Risk estimates.

The VaR estimate is found from the probability distribution of the expected returns. This implies that one needs to make assumptions concerning the actual form of the expected return distribution. This can be done by assuming that the distribution of the expected returns equals the empirical distribution based on past observations or by assuming that the returns are drawn from a specific statistical distribution. The exact form of these analytical distributions is determined by various parameters, estimated using past data, and which have more recently also allowed for the use of conditioning methodologies such as GARCH.

A parametric approach has been the preferred method, since it enables simple conversion to take place (between quantiles and time horizons), and is hence more pragmatic under the framework of the Basle Committee. It also enables conditionality in the data to be easily 


\section{Chapter 3: Fat tails in financial risk management}

incorporated into the $\mathrm{VaR}$ estimate, making forecasts of $\mathrm{VaR}$ more appropriate. The crucial assumption therefore for an accurate estimation of the $\mathrm{VaR}$ is that the distribution in the left tail, reflecting the negative returns, is well represented by the specified distribution. Any discrepancy between the parametric distribution and the empirical distribution can result in large errors in the estimation of the Value-at-Risk.

For simplicity and convenience, asset returns are often assumed to be normally or lognormally distributed. However the return distributions on many assets have been shown to exhibit fatter tails than the normal distribution ${ }^{15}$. This means that the assumption of normality results in an underestimation of the VaR on moving further into the tails. It is the exact nature of this extra mass in the tails of the distribution, which is crucial when trying to capture the VaR of an asset. Other fatter tailed distributions such as Pareto and sumstable distributions have in the past proved difficult to implement. The normal distribution has therefore been retained as the most convenient proxy for an assetís actual distribution. However, the fatter the tails of the asset return distribution under consideration, the larger the discrepancy with the normal distribution, and the larger the errors made in VaR estimation: errors, which become magnified for the million-dollar positions, which mutual funds for example typically hold.

There is thus a need for simple methodologies to estimate VaR, which capture the tail fatness apparent in return distributions. In this chapter we present such a simple technique.

15 See among others Fama and Roll (1968), Rogalski and Vinso (1978), Boothe and Glassman (1987), Taylor (1986), Jansen and de Vries (1991), Loretan and Phillips (1994), and Huisman, Koedijk, Kool, and Palm (1998, 2000). 
We show that VaR-x estimates, VaR estimates obtained from assuming a Student-t distribution to fit the empirical distribution, are better able to capture the extra risk involved, for distributions exhibiting a higher probability of large negative returns. Since we are looking at downside risk, we are interested in the negative returns associated with the left tail of the distribution. The tail parameter of the Student-t distribution, reflected by its number of degrees of freedom, is set equal to the tail-index for the left tail, and is a direct measure of the amount of fatness in the tail of the returnís distribution. This method offers many advantages with respect to using the normal distribution. Firstly, fat tails are captured. Secondly, focussing only on the left tail means that we do not need to assume distributions are symmetric. The tail fatness may vary between the two tails of the return distribution and hence allows for the possibility for skewness in the distribution. This provides us with a simple, more accurate estimator than would otherwise be obtained from assuming normality.

One attempt to capture the extra probability mass in the tails has been to estimate a Generalised Autoregressive Conditionally Heteroskedastic (GARCH) process. The unconditional distribution of a GARCH process does reveal fatter tails; however it has been shown that the distribution of conditional residuals is still non-normal, see Bollerslev (1987). This results in the Value-at-Risk still being underestimated at high quantiles for fat tailed assets. The appealing feature however of incorporating conditional volatility is that it allows for a changing distribution over time. Implementing this into a VaR framework 


\section{Chapter 3: Fat tails in financial risk management}

means that the VaR estimates are made conditional. This is done in JP Morganís Risk Metrics for example, and VaR-x can easily be adjusted to capture conditional volatility ${ }^{16}$.

The plan of this chapter is as follows. Value-at-Risk and VaR-x are introduced in the following section. The data used and the results are presented in section 3.2 and 3.3 respectively. Conclusions are then drawn in the final section.

\subsection{VALUE-AT-RISK METHODOLOGY}

Exposure to downside risk can be summarised in a single number by an estimate of the Value-at-Risk. This is formally defined by Jorion (1996) as ì the worst expected loss over a target horizon within a given confidence levelî. Following Jorion we define $\mathrm{W}_{0}$ as the initial investment and $\mathrm{R}$ as the expected return over the target horizon. $\mathrm{W}^{*}$ is defined as the lowest portfolio value at the given confidence level c, i.e. the value of the portfolio should not fall below $\mathrm{W}^{*}$ with probability $\mathrm{c}$. $\mathrm{VaR}$ is defined as the dollar loss relative to the expected mean value of the portfolio:

$$
V a \mathrm{R}=E(W)-W^{*}
$$

Defining $\mathrm{R}^{*}$ as the expected return associated with the portfolio value $\mathrm{W}^{*}$;

$$
W^{*}=W_{0}\left(1+R^{*}\right)
$$

16 See Koedijk and Pownall (1998) for an implementation of a conditional VaR-x approach and comparison of results to RiskMetrics using data on Asian emerging markets. 
Gives us the VaR measured as the dollar loss relative to the mean, where $\mu$ is the expected return on the portfolio for the target horizon;

$$
V a \mathrm{R}=-W_{0}(\mathrm{R} *-\mu)
$$

The crux of being able to provide an accurate $\mathrm{VaR}$ estimate is in estimating the cutoff return $\mathrm{R}^{*}$. In this paper, we focus on the cut off return estimated using historical data, and thereby assume that these are representative for the expected return measure.

The statistical methods developed to best estimate these cutoff returns can be divided into two types: parametric and non-parametric. The most obvious non-parametrical approach uses the historical distribution itself to compute an empirical estimate of the VaR directly. In the parametric case one tries to fit the historical distribution by a statistical distribution whose characteristic parameters are derived from the historical data. We shall therefore briefly review the standard ways to estimate VaR before presenting the methodology behind VaR-x. The crucial difference between VaR and VaR-x is that the latter incorporates the tail fatness apparent in financial returns into the VaR estimate, thereby improving the quality of the estimates in a simple and efficient way.

\subsubsection{METHODS TO ESTIMATE THE CUT-OFF RETURN AND VAR}

The cut-off return is defined as the worst possible realisation $\mathrm{R}^{*}$ for a confidence level $\mathrm{c}$, and is found from the following integral for the distribution of expected returns $f(r)$ : 


$$
1-c=\int_{-\infty}^{R^{*}} f(r) d r
$$

\subsubsection{EMPIRICAL VAR}

Empirical VaR involves determining the point $\mathrm{R}^{*}$ from a histogram of the empirical distribution based on historical returns. $\mathrm{R}^{*}$ is that point below which the fraction $1-\mathrm{c}$ of the returns are. This number is then plugged into (3.3) to get the empirical VaR estimate.

The empirical VaR measure has some serious disadvantages to both financial institutions and regulators. In order to obtain accurate estimates a large data sample of the empirical distribution is required. The VaR estimate is therefore subject to the frequency and length of the data sample. A further drawback is the inability to allow for conditionality of the parameters over time. To overcome these flaws a parametric approach, such as the normal approach, is often adopted. Since the distribution is approximated by a parametric distribution parameters can be allowed to change over time. Estimation risk on the VaR estimate itself is also reduced, particularly for higher quantiles ${ }^{17}$. Furthermore, the parametric approach has the advantage of not being dependent on the chosen quantile, facilitating the ease with which comparisons, between the VaR estimates across various

17 See Jorion (1996) and Kupiec (1995) for a greater insight into the recognition of estimation error in VaR estimates. 
institutions, can be made. Parametric conversion however, will only hold in practice if the parametric approach accurately reflects the distribution at all quantiles in the tail. Indeed it has been the case that institutions have notoriously chosen confidence levels and time horizons to suit them. Vital is therefore the choice of parametric distribution.

\subsubsection{NORMAL VAR}

The simplest parametric approach is to assume that the expected returns are normally distributed with the mean and variance estimated using past data on returns. VaR estimates are then obtained by equating $\mathrm{f}(\mathrm{r})$ in equation (3.4) to the pdf of the normal distribution. The simplicity of this method also explains its popularity. However, to obtain accurate VaR estimates for higher confidence levels, say more that $95 \%$, the parametric distribution should correctly approximate the distribution in the tails. Since it is commonly known that the distributions of returns on financial assets often exhibit fatter tails than the normal distribution, one could expect a large discrepancy to exist between the tails of the normal distribution and the tails of the actual distribution. Such a discrepancy could lead to serious errors in VaR estimates. These estimates could thus be improved upon by incorporating tail fatness; one such technique that incorporates the fat tails is VaR-x, proposed in the following section. 


\subsubsection{VAR-X}

The evidence that distributions of returns on financial assets have fatter tails than indicated by the normal distribution has meant that the normal approach underestimates the true Value-at-Risk at high quantiles. It therefore becomes apparent that in order to capture the full risk from fat tailed assets a parametric distribution that is fatter in the tails should be used. The Student-t distribution, which also nests the normal distribution, is one obvious choice to model $\mathrm{f}(\mathrm{r})$ in equation (3.4); however its parameterisation has proved tedious and inconsistent in the past ${ }^{18}$.

The Student-t is a distribution that exhibits fatter tails than the normal distribution. The amount of tail fatness is reflected in the number of degrees of freedom. In order to capture tail fatness correctly one should correctly specify the exact number of degrees of freedom to be used. This has proved to be difficult for exchange rate returns (see Boothe and Glassman (1987), and Huisman et al. (1998)); however recent advances in Extreme Value Theory makes the issue less complex.

Extreme Value Theory looks specifically at the distribution of the returns in the tails. Since VaR focuses predominantly on this area in the tail, Extreme Value Theory can bring some valuable insight into improving $\mathrm{VaR}$ estimation ${ }^{19}$. The tail fatness that a tail of a

\footnotetext{
18 Alternative distributions to capture the tail fatness are, for example, Pareto and Sum-Stable distributions or a mixture of two Normals.

19 See Danielson and de Vries (1997).
} 
distribution exhibits is reflected by the tail index. It measures the speed with which the tail under consideration approaches zero. The fatter the tail the slower the speed and the lower the tail index given. A nice feature of the tail index is that it equals the number of moments that exist for a distribution. For example, a tail index estimate equal to 2 reveals that both the first and second moments exist, i.e. the mean and the variance, but that higher moments are infinite. All moments exist for the normal distribution, so that its tail index equals infinity by definition. Here lies also the link with the Student-t distribution. The number of its degrees of freedom reflects the number of existing moments, and the tail index can thus be used to set the number of degrees of freedom ${ }^{20}$.

To obtain tail index estimates we use the estimator presented by Huisman et al (2000). Unlike other tail index estimators, the Huisman et al. estimator is shown to produce almost unbiased estimates in relatively small samples. This provides us with a superior estimator than those previously used, and, as we see later, allows us to obtain robust tail index estimates from a yearly sample of daily data. Danielson and de Vries (1997) also use extreme value theory to obtain $\mathrm{VaR}$ estimates; however their approach has the drawback that an extremely large sample of data is required ${ }^{21}$.

Specifying $\mathrm{k}$ as the number of tail observations, and ordering their absolute values as an increasing function of size, we obtain the tail estimator proposed by Hill (1975). This is denoted below by $\gamma$ and is the inverse of the tail index $\alpha$. Let $\mathrm{X}_{\mathrm{i}}$ be the $\mathrm{i}^{\text {th }}$ increasing order statistic, i.e. $X_{i} \geq X_{i-1}$, based on the absolute values of the observations.

20 Huisman et al. (1998) use this method to fit the unconditional distribution of exchange rate returns. 


$$
\gamma(k)=\frac{1}{k} \sum_{j=1}^{k} \ln \left(x_{n-j+1}\right)-\ln \left(x_{n-k}\right)
$$

Following the methodology of Huisman et al. (2000), we can use a modified version of the Hill estimator to correct for the bias in small samples. The bias of the Hill estimator stems from the fact that the bias is a function of the sample size. A bias corrected tail index is therefore obtained by observing the bias of the Hill estimator as the number of tail observations increases up until $\kappa$, where $\kappa$ is equal to half of the sample size:

The optimal estimate for the tail index, is the intercept $\beta_{0}$ in the following equation (3.6). The $\alpha$ estimate is just the inverse of this estimate, and it is this estimate of the tail index that we shall use in order to parameterise the Student-t distribution.

$$
\gamma(k)=\beta_{0}+\beta_{1} k+\varepsilon(k), k=1 \ldots \kappa
$$

The procedure to obtain the VaR-x estimates is therefore as follows. First, the tail index referred to by $\alpha$ is estimated using the Huisman et al. estimator for the left tail of the empirical return distribution. The focus on the left tail directly reflects the downside risk. Furthermore, the mean $\mu$ and the variance $\sigma^{2}$ of the return distribution are estimated. In the second step, the tail index estimate $\alpha$ is then used to equate the number of degrees of freedom in the Student- $t$ distribution. Read the value $\mathrm{S}^{*}$ off the standard Student- $\mathrm{t}$

21 Danielson and de Vries (1997) typically require about 100000 observations, obtained from high frequency data. 
distribution with $\alpha$ degrees of freedom using appropriate tables provided in standard textbooks (see Bain and Engelhardt for example) or statistical software. This value then needs to be converted, since the standard Student-t distribution with $\alpha$ degrees of freedom has a pre-set mean equal to zero and a variance equal to $\alpha /(\alpha-2)$. The value $S^{*}$ is then transformed into the real cutoff return $R^{*}=-\theta S^{*}+\mu$, where $\theta$ is a scale factor equal to:

$$
\theta=\frac{\sigma}{\sqrt{\frac{\alpha}{\alpha-2}}}
$$

The value $\mathrm{R}^{*}$ then equals the cutoff return needed to calculate the VaR-x measure for the confidence level c. Plugging the expression for $\mathrm{R}^{*}$ into (3.3) we obtain the VaR-x estimate for the VaR relative to the mean $\mu$;

$$
V a \mathrm{R}-x=W_{0} \theta S *
$$

In the following sections we shall apply all the above techniques to calculate the VaR for an \$100 million investments in both US stocks and bonds.

\section{2 DATA}

We use data from US Stock and Bond indices from January 1980 until August 1998, using bi-weekly returns to provide results that can easily be set against the 10 day, regulatory framework adopted by the Basle Committee. The use of two different assets exhibiting 


\section{Chapter 3: Fat tails in financial risk management}

different tail index alphas will enable us to gauge the effect on the Value-at-Risk estimates from a variation in tail fatness.

We use data on the S\&P 500 Composite Return Index for the US and the 10 Year Datastream Benchmark US Government Bond Return Index, both obtained from Datastream. The bi-weekly data excludes the crash of October 1987, so that we can estimate the Value-at-Risk consistent with normal market conditions. Summary statistics are presented using lognormal returns for the sample of stock and bond returns in Table 3.1. Over the period, stocks have had an average return of $17.33 \%$ per annum, nearly twice the $10.25 \%$ return on government bonds. The volatility was however much lower for US government bonds, with the variance around a third of that prevailing on the S\&P 500 . Both assets appear to exhibit significant skewness as well as excess kurtosis. According to the kurtosis statistic the extra probability mass in the tail areas of the stock returns appears to be high, and since the distributions appear skewed, the two tails may differ dramatically. For the VaR-x estimates we hence take any skewness in the tails into account by taking the tail index estimator of the left tail only. The effect on the VaR depends on the exact structure of the distribution of negative returns. 


\section{TABLE 3. 1 SUMMARY STATISTICS FOR STOCKS AND GOVERNMENT}

\section{BOND RETURNS}

This table contains the statistics on the S\&P 500 Composite Return Index and the 10 Year Datastream US Benchmark Government Bond Index for the period January 1980 until August 1998 using 486 biweekly total returns. The alpha estimate is calculated using a modified version of the Hill estimator for the tail indexes and is presented for the left tail.

$\begin{array}{cc}\text { S\&P 500 } & \text { US 10 YEAR } \\ \text { COMPOSITE } & \text { GOVERNMENT BOND } \\ \text { RETURN INDEX } & \text { RETURN INDEX }\end{array}$

Annual Mean \%

17.329

10.247

Max Return

0.153

0.089

Min Return

$-0.183$

$-0.061$

Annual St Deviation

0.146

0.086

Annual Variance

0.021

0.007

Skewness

$-0.641$

0.503

Kurtosis

9.399

Gamma Left Tail

0.233

0.143

Standard Error

0.050

0.030

Alpha Left Tail

4.285

7.009

Observations

118 


\section{Chapter 3: Fat tails in financial risk management}

The degree of fat tailedness is estimated in terms of $\alpha$, calculated using the estimator developed by Huisman et al. (2000). From the gamma estimates of the left tail (standard errors given below) the alpha estimates are 4.29 and 7.01 for the stock and bond returns respectively. For normally distributed returns the alpha estimate tends to infinity, so we can see that both distributions exhibit a fatter left tail than the normal. Due to this leptokurtosis the frequency of large negative returns is greater than reflected by the normal distribution, hence the greater the downside risk the fatter the tails, with the equity returns exhibiting more downside risk than the bonds.

\section{3 VALUE-AT-RISK ESTIMATES}

Value-at-Risk by definition should be highly sensitive to the degree to which the distribution is fat tailed: the fatter tailed the distribution the higher the Value-at-Risk for a given confidence level. As we have seen stocks have more downside risk than bonds, represented by a lower alpha estimate for the left tail index, and thus have a higher than normal probability of extreme returns. Thus for higher confidence levels, we would expect an empirical VaR estimate to be larger than that predicted from using the parametric approach assuming normality. The higher the confidence level and thus the quantile chosen for the VaR estimate the greater the effect of extreme values in the assetís return distribution. This has the important implication that the existence of a fat tailed return distribution implies that at high confidence levels the parametric-normal VaR underestimates the exposure to market risk, with the difference likely to grow for higher confidence levels chosen and fatter tails. 
To see by how much the estimates for Value-at-Risk are affected by the evidence that stocks have a lower alpha tail index estimate than the bonds, we compute the VaR estimates using the various approaches as discussed in section 2. In Table 3.2 the Value-atRisk is estimated for a $\$ 100$ million investment in the two assets, using both the parametric-normal approach (equally weighted moving average method for calculating volatility) and the empirical approach. We can see how the VaR estimates increase, the higher the confidence level taken. The structure of the difference between the empirical and the parametric-normal VaR estimates is indeed what would be expected for a fat tailed distribution. For the S\&P 500 Composite we see that for low probability levels the distribution exhibits a so called thin waist, since the parametric-normal VaR is larger than the empirical VaR. Moving further into the tails the VaR estimate assuming normality becomes smaller than the empirical VaR. This means that at low probability levels the parametric-normal VaR overestimates the VaR and then as we move to higher probability levels the parametric-normal approach underestimates the VaR. 
Chapter 3: Fat tails in financial risk management

\section{TABLE 3. 2 COMPARISON OF VALUE-AT-RISK ESTIMATES}

The Value-at-Risk Estimates have been calculated for the two asset classes using the Empirical Approach (historical data) and both Parametric approaches. The Normal VaR estimates assume Normally distributed returns, whereas the VaR-x estimates assume a fatter tailed distribution denoted by the Student-t, and use the alpha estimates for the left tail as given in Table 3.1. The relative VaR estimates, expressed in millions of dollars, have been calculated for a position of $\$ 100$ million in the particular asset, and for a range of confidence levels.

S\&P 500

US 10 YEAR

COMPOSITE

GOVERNMENT BOND

RETURN INDEX

RETURN INDEX

Confidence Level Empirical Normal Student-t Empirical Normal Student-t $\begin{array}{lllllll}\text { (Left Tail) } & \text { VaR } & \text { VaR } & \text { VaR-x } & \text { VaR } & \text { VaR } & \text { VaR-x }\end{array}$

$\begin{array}{ccccccc}\mathbf{9 5} & 4.3288 & 4.7176 & 4.3563 & 2.5347 & 2.7505 & 2.6812 \\ \mathbf{9 5 . 5} & 4.6174 & 4.8626 & 4.5463 & 2.5986 & 2.8350 & 2.7819 \\ \mathbf{9 6} & 4.7584 & 5.0211 & 4.7613 & 2.6912 & 2.9274 & 2.8942 \\ \mathbf{9 6 . 5} & 4.9716 & 5.1967 & 5.0086 & 2.7648 & 3.0298 & 3.0212 \\ \mathbf{9 7} & 5.3585 & 5.3943 & 5.2993 & 2.9283 & 3.1450 & 3.1678 \\ \mathbf{9 7 . 5} & 5.7897 & 5.6214 & 5.6512 & 3.0463 & 3.2774 & 3.3414 \\ \mathbf{9 8} & 5.9831 & 5.8903 & 6.0950 & 3.1674 & 3.4342 & 3.5546 \\ \mathbf{9 8 . 5} & 6.3505 & 6.2240 & 6.6909 & 3.2729 & 3.6287 & 3.8315 \\ \mathbf{9 9} & 7.5190 & 6.6722 & 7.5825 & 3.5503 & 3.8900 & 4.2273\end{array}$


The magnitude of the error from using the normality assumption is a reflection of the amount of tail fatness, which of course is much more significant for the stock price index. The extent of the discrepancy from using the assumption of normality for the S\&P 500 and the Government Bond Index is depicted in Figure 3.1.

\section{FIGURE 3. 1 VALUE-AT-RISK ESTIMATES}

The graph depicts how much the Parametric-Normal VaR estimates differ from the Empirical VaR estimates for the two assets over a range of confidence levels. The Parametric-Normal approach assumes Normally distributed returns and the Empirical Approach uses the observed frequency distribution. The difference is the error generated by using the assumption of Normally distributed returns and is estimated for a $\$ 100$ million position in the particular asset.

\section{Value at Risk}

Empirical VaR - Parametric Normal VaR

S\&P 500 Composite Return Index and the 10 YearDatastream Benchmark US Government Bond Return Index: \$100 Million Position

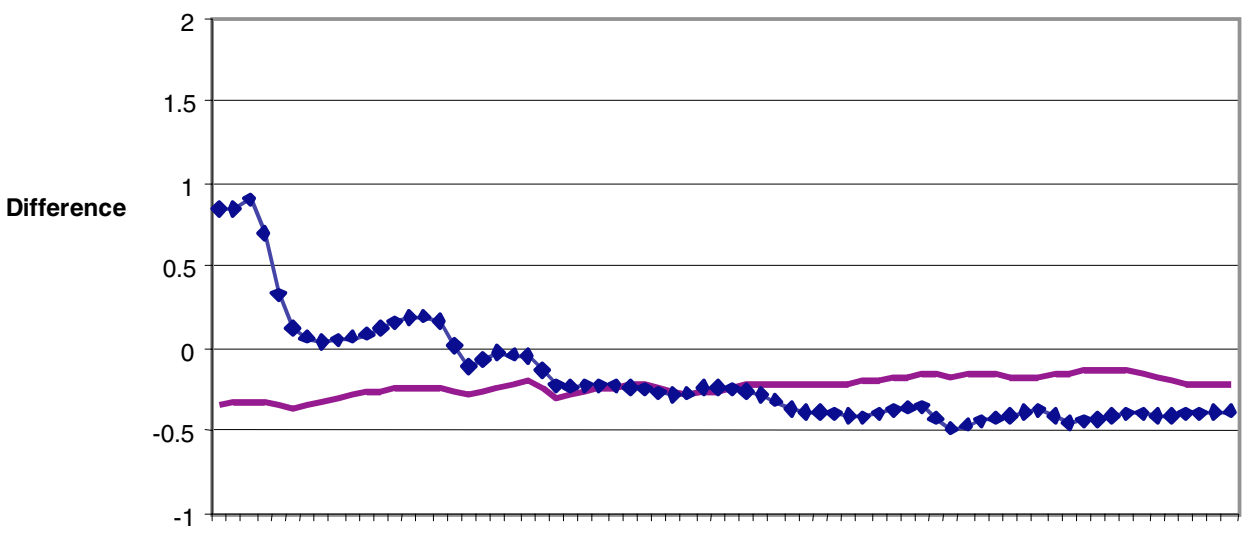

98.998 .598 .197 .797 .396 .996 .596 .195 .795 .394 .994 .594 .193 .793 .392 .992 .592 .1

\section{Quantile}

$\checkmark$ S\&P 500 Composite Return Index

10 Year Datastream Benchmark US Govn. Bond Index 


\section{Chapter 3: Fat tails in financial risk management}

As predicted the difference is larger for stocks, whose return distribution exhibits a fatter tail (lower alpha estimate), and becomes much larger for confidence intervals above the $96 \%$ level. In the example the assumption of normality means that the $\mathrm{VaR}$ is underestimated above the $96 \%$ level, and greatly underestimated at the $99 \%$ level. We therefore conclude that the assumption of normality appears inappropriate for estimating VaR at high quantiles for a distribution with an alpha estimate of around 4.

In Table 3.2 we indeed see that taking a $\$ 100$ million position in the S\&P 500 Composite Return Index generates a relative VaR estimate at the $95 \%$ probability level of $\$ 4.72$ million, using the parametric assumption of normality, compared to the $\$ 4.33$ million using the empirical distribution. The average bi-weekly return is $\$ 0.67$ million, and the Value-at-Risk is stated relative to this mean. This means that assuming normally distributed returns, $95 \%$ of the time we would not expect to achieve a bi-weekly loss of more than $\$ 4.72$ million. However at the $99 \%$ confidence interval the VaR becomes $\$ 6.67$ and $\$ 7.52$ million respectively. The large discrepancy of over $\$ 0.75$ million between the two approaches shows just how important it is to find as accurate a measure as possible for the VaR. Indeed since the $99 \%$ level is the level required by the Basle Committee then it becomes apparent just how inappropriate the assumption of normality in the tails is.

The VaR-x estimates, which incorporate the fat tails, are also given in Table 3.2. The $\alpha$ estimates from Table 3.1 are used to parameterise the Student-t distribution. For the S\&P 500 Composite Return Index the VaR-x estimates provides a much more accurate estimate 
when compared to the empirical distribution for the whole range of quantiles, than the parametric-normal VaR.

\section{FIGURE 3.2 VAR-X FOR THE S\&P 500 COMPOSITE RETURN INDEX}

The graph depicts how the VaR-x estimates, using the Student-t distribution, compare with the ParametricNormal VaR estimates and the Empirical VaR estimates for the S\&P 500 over a range of confidence levels. The VaR-x uses the modified Hill estimator for the tail index as the parameter in the Student-t distribution, the Parametric-Normal approach assumes Normally distributed returns and the Empirical Approach uses the observed frequency distribution. The difference is given in million dollars for a position of \$100 million in the particular asset.

\section{Relative Value at Risk: S\&P 500 Composite Return Index Bi-Weekly data (\$100 Mill.)}

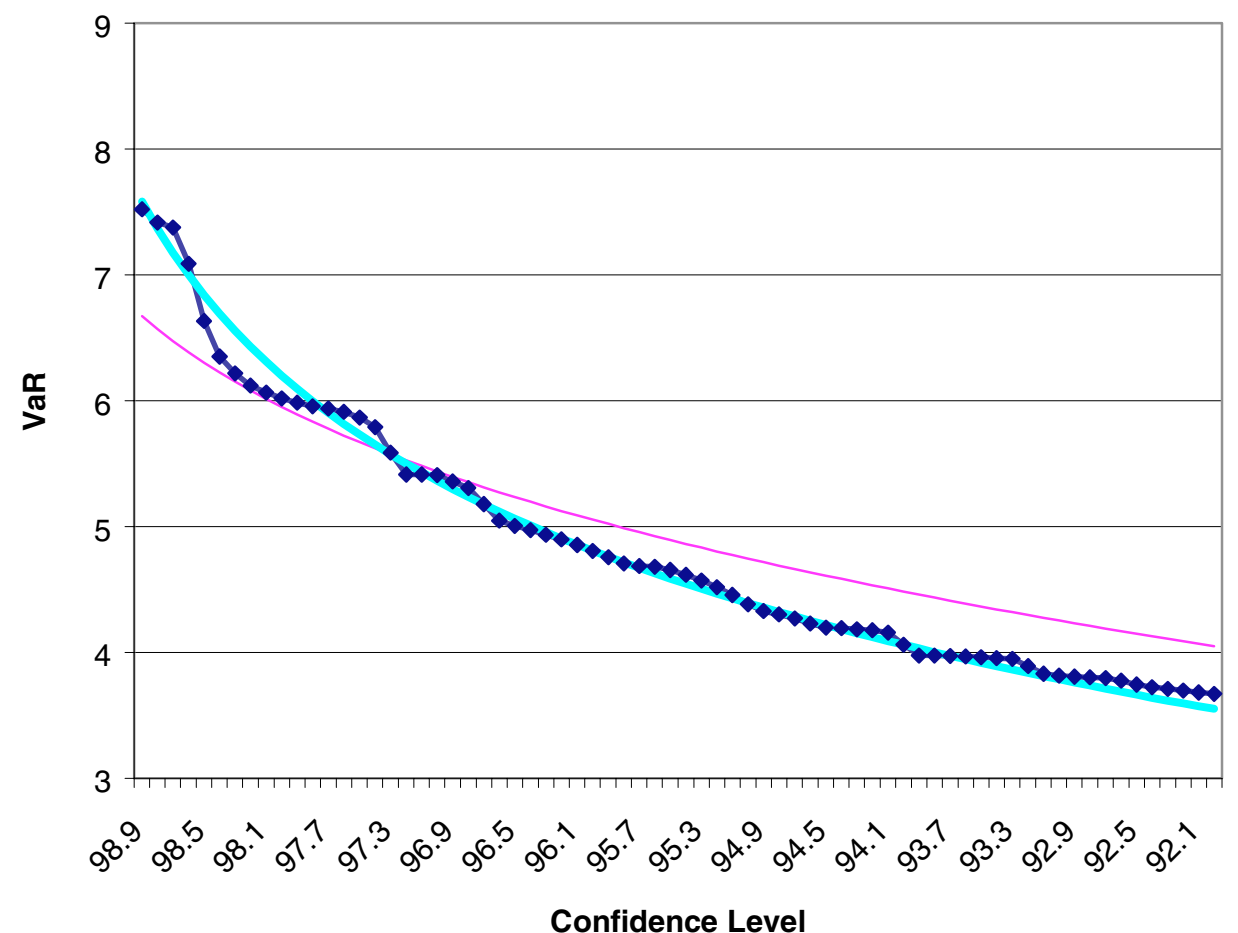

$\rightarrow$ Empirical VaR $\longrightarrow$ Normal VaR $\longrightarrow$ VaR-x 
Chapter 3: Fat tails in financial risk management

\section{FIGURE 3. 3 VAR-X FOR THE 10 YEAR US GOVERNMENT BOND INDEX}

The graph depicts how the VaR-x estimates, using the Student-t distribution, compare with the ParametricNormal VaR estimates and the Empirical VaR estimates for the US Government Bond Index over a range of confidence levels. The VaR-x uses the modified Hill estimator for the tail index as the parameter in the Student- $t$ distribution, the Parametric-Normal approach assumes Normally distributed returns and the Empirical Approach uses the observed frequency distribution. The difference is given in million dollars for a position of $\$ 100$ million in the particular asset.

\section{Relative Value at Risk: Datastream Bond Index Bi-Weekly data}

(\$100 Mill.)

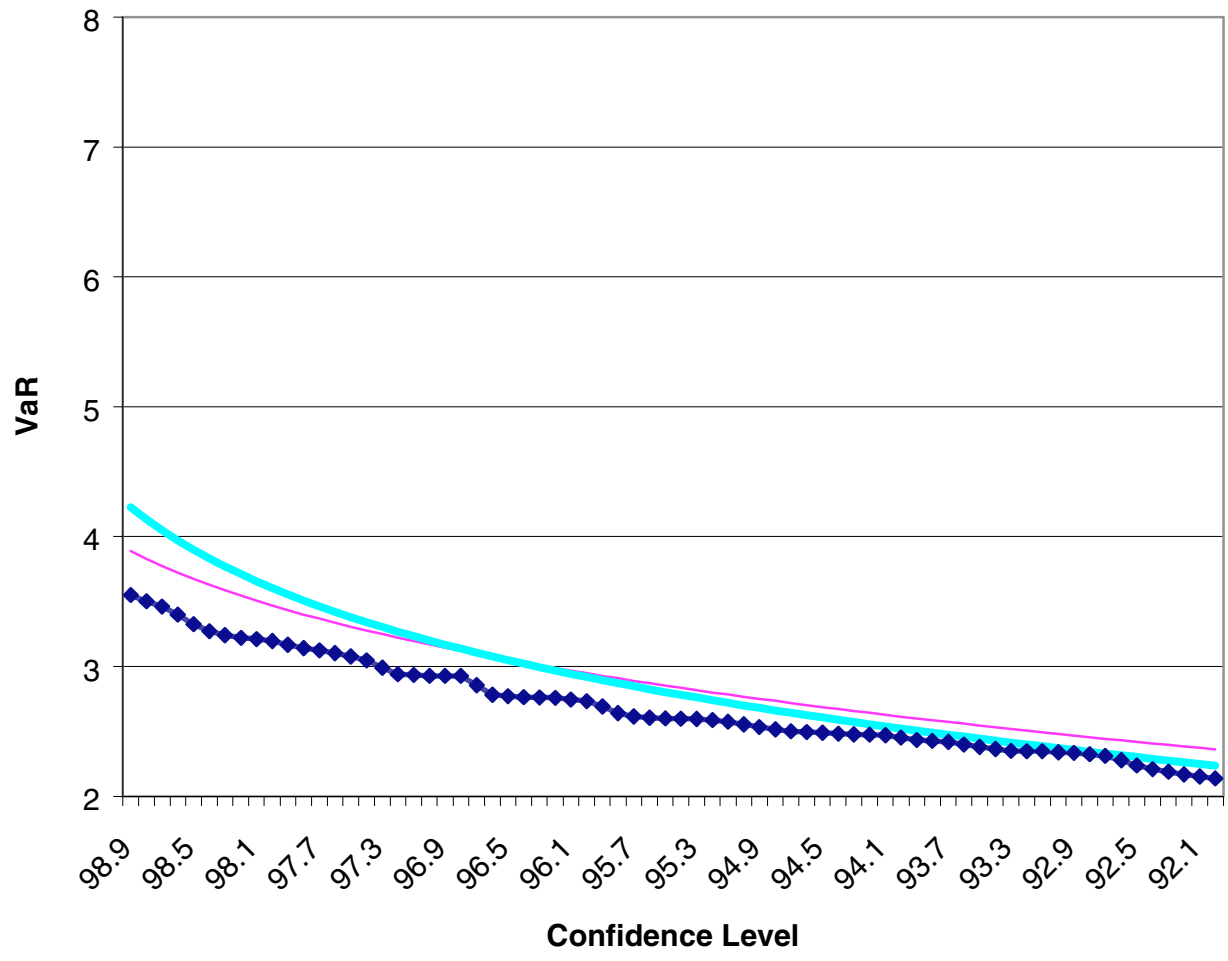

$\multimap$ Empirical VaR $\longrightarrow$ Normal VaR VaR-x 
This is illustrated in Figure 3.2, where all three estimates for a range of confidence levels are plotted. For the US Government Bond Index, as can be seen in Figure 3.3, the difference between the two parametric approaches is much less, reflecting that an alpha estimate of around 7 already gives similar results to those under normality.

This approach therefore provides us with an estimator, which more accurately reflects the VaR estimates for the whole range of confidence levels, and is thus a more accurate estimate for assessing the downside risk as measured by Value-at-Risk. We have seen that the estimator performs well for a range of quantiles up to and including the $99 \%$ level, and therefore allows for simple parametric conversion to be adhered to. Indeed time aggregation for various holding periods is merely a simple extension to the framework, so that the estimates provided can easily be converted for different quantiles and time horizons, as required by the regulatory bodies.

To see how forecasts of the two approaches perform over time, we perform the following out of sample test. We have estimated the rolling 10-day absolute VaR forecasts, at the 99\% level for the S\&P 500 Composite return Index, using both the parametric-normal and VaR-x methods. These forecasts, using daily returns, are plotted in Figure 3.4 against the actual rolling bi-weekly returns, of which some appear to look like multiple returns.

From Table 3.3 we can see that the parametric-normal approach provides VaR forecasts within a $99 \%$ confidence interval which are exceeded $1.99 \%$ of the time. Since a $99 \%$ 


\section{Chapter 3: Fat tails in financial risk management}

confidence level requires the actual returns to exceed it only $1 \%$ of the time the VaR-x forecasts have performed much better, with the forecast only being exceeded $0.94 \%$ of the

\section{Figure 3. 4}

\section{Rolling VaR-x and Parametric-Normal VaR estimates}

The graph shows how the forecasts of the VaR-x estimates, using the Student-t distribution, compare with forecasts from using the Parametric-Normal VaR approach for the S\&P 500 Composite Return Index. We have used rolling observations of daily data, over the period January 1994 until August 1998 using 1216 rolling BiWeekly total returns, to provide forecasts of the Value-at-Risk at the $99 \%$ confidence level. The forecasts are based on yearly samples of daily data, and the alpha estimate is calculated for the left tail using a modified version of the Hill estimator.

\section{Biweekly Forecast of 99\% VaR for the S\&P 500 (252 obs)}

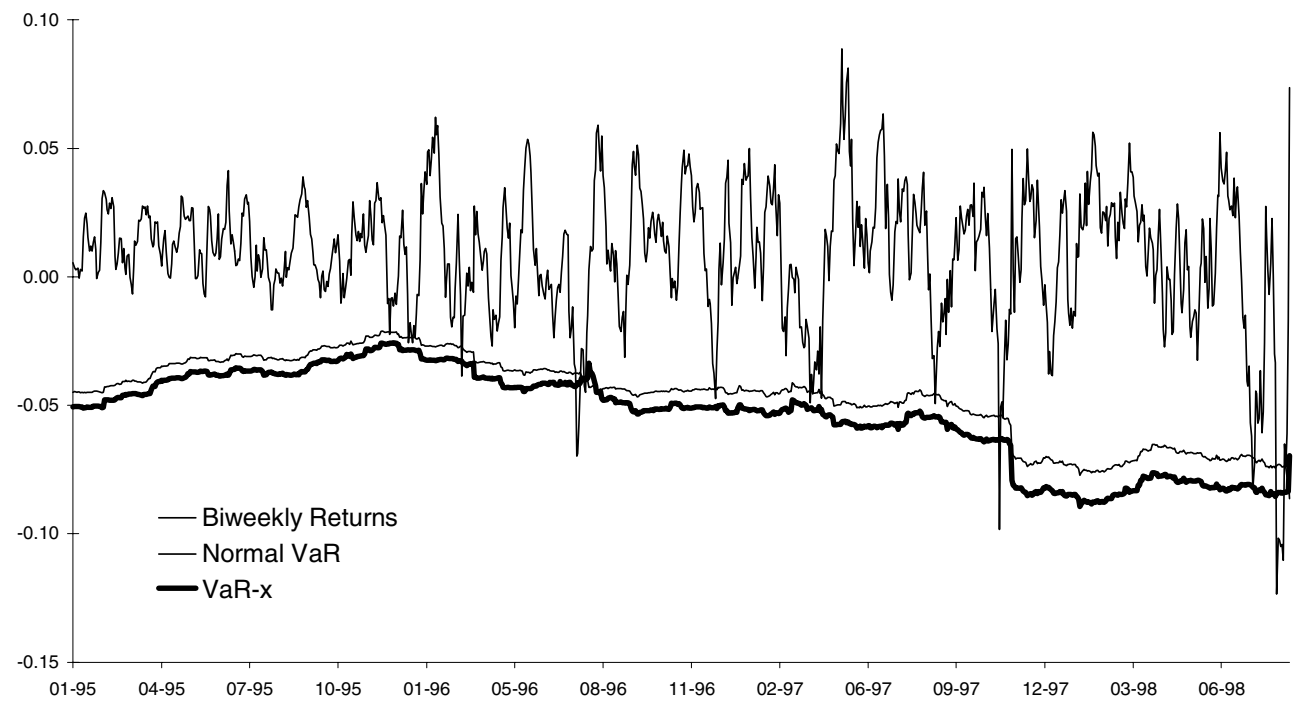


time. The consistently greater VaR-x forecasts provide evidence of the stability of $\alpha$, and hence the VaR-x estimates. The VaR-x method using data on the Government Bonds showed a slight improvement yielding $1.15 \%$ compared with $1.47 \%$ for the parametricnormal approach.

\section{TABLE 3. 3 EXCEEDANCE STATISTICS FOR ROLLING BI-WEEKLY RETURNS}

This table contains the statistics on the S\&P 500 Composite Return Index and the 10 Year Datastream US Benchmark Government Bond Index for the period January 1994 until August 1998 using 1216 rolling bi-Weekly total returns. The forecasts are based on yearly samples of daily data (252 observations), and the alpha estimate is calculated for the left tail using a modified version of the Hill estimator.

EXCEEDANCE OF VAR AT 99\% CONFIDENCE LeVEL THEORETICAL NORMAL VAR VAR-X

\section{NUMBER OF EXCEEDANCES}

S\&P 500 Composite Return

$$
\text { Index }
$$

9.64

19

US 10 Year Government Bond

Return Index

9.64

14

PeRCENTAGe OF EXCEEDANCES

S\&P 500 Composite Return

Index $\quad 1.00 \% \quad 1.990 \% \quad 0.942 \%$

US 10 Year Government Bond

Return Index

$1.00 \%$

$1.466 \%$

$1.152 \%$ 


\section{Chapter 3: Fat tails in financial risk management}

These results clearly show that the VaR-x method provides a more accurate estimate for the Value-at-Risk than the parametric-normal approach, illustrating the importance of including the tail fatness into the $\mathrm{VaR}$ estimate. Indeed we see that by including a parameter for the distributionís fat tailedness the estimate assesses the downside risk much more adequately than the assumption of normally distributed returns in the tails. The structure of the VaR-x parametric approach compared with that of the normal provides us with the phenomena of a larger Value-at-Risk as we move further into the tails. Indeed as the normal distribution is nested in the Student-t distribution, as the alpha estimates become larger (less fat tailed) then the VaR estimates will converge. This therefore provides us with a consistent parametric approach to modeling the additional downside risk that fat tailed assets have, which can easily be extended to allow for further conditionality in the data ${ }^{22}$.

\section{4 CONCLUDING REMARKS}

It is widely known that the distributions of financial asset returns exhibit fatter tails than the normal distribution. This implies that the downside risk of a portfolio containing fat tailed assets, as measured by Value-at-Risk is underestimated when VaR is estimated with the assumption of normally distributed returns. Furthermore this suggests that parametric conversion, for different confidence levels as adhered to by the regulatory framework of the Basle Committee will provide inaccurate estimates of the VaR. It is no wonder, that it

22 See Koedijk and Pownall (1998). 
has been necessary to ëad hoclyí multiply the VaR by 3 to provide a larger, more representative, number for the Basle capital requirements. It would be preferable to have a more accurate measure reflecting the true risk from extreme returns, and the avoidance, or reduction at least, of the Basle Multiplication factor. In this chapter we present such a measure: VaR-x.

This methodology provides us with a simple approach to finding an accurate estimator for the VaR. The tail fatness apparent in financial returns is incorporated more accurately into the VaR-x estimator by using the Student-t distribution as a proxy for the distribution of future returns. We show that for both US stocks and bonds the VaR-x estimates reflect the true downside risk, apparent in financial returns, much better than those from the standard VaR estimators. The approach is easily extended to include further time varying parameters, and hence the implications for risk management seem tremendous. Certainly the move towards building portfolios which exploit these departures from normality (see Bekaert, Erb, Harvey and Viskanta 1998) will only serve to underline the vital importance of including an additional measure for the downside risk into the risk management techniques of the future. 
Chapter 4: Measuring credit spread risk: Incorporating the tails

\section{CHAPTER 4 MEASURING CREDIT SPREAD RISK: INCORPORATING THE TAILS ${ }^{23}$}

It is widely known that the small but looming possibility of default renders the expected return distribution for financial products containing credit risk to be highly skewed and fat tailed. In this chapter we apply recent techniques developed for incorporating the additional tail risk faced from changes in swap spreads into estimates of credit spread risk. Using data from the US, UK, Germany, and Japan, we find that the risk faced from large spread widenings and tightenings is grossly underestimated. Estimation of swap spread risk is dramatically improved when the severity of the fat tails is measured and incorporated into current estimation techniques.

Credit risk is the risk resulting from uncertainty in a counterparty's ability or willingness to meet its contractual obligations, for example default on loan payments, pre-settlement

This chapter is based on R.A. Campbell and R. Huisman, 'Measuring Credit Spread Risk: Incorporating the Tails' Working Paper (2000). 
credit risk on a swap contract, or settlement credit risk on a forward contract. Credit risk management has become an increasingly important area of financial risk management, which is highlighted by the enormous surge in credit derivatives, a risk management tool for controlling credit risk. A survey by the British Bankers Association (BBA) estimated the global credit derivatives market in 1998 to be $\$ 350$ billion, and by April 1999 the market had already grown to around $\$ 480$ billion $^{24}$; however estimates for this year have mushroomed to an incredible $\$ 900$ billion. Recent global financial crisis, the need for credit protection, as well as the potential to enhance loan-based credit portfolio yields and the returns on bank capital have spurred demand for credit derivatives.

Accurate assessment of credit risk depends on methods to accurately measure and control the potential or expected losses resulting from default. This includes estimation of the credit exposure (financial loss that would occur should the client default), the probability of default, and the fraction of the market value being recovered at default. Measuring credit risk therefore integrates market risk, estimation of default probabilities, and recovery rates. Credit spreads, the difference between the risky bond and a risk-free alternative, should therefore reflect the amount of credit risk faced. These spreads change over time due to, for example, varying market conditions, changes in the credit ratings of issuers, or changes in the expectations regarding the recovery rate.

Traditional credit risk models assume that expected changes in spreads are normally distributed, but empirical evidence shows that they are more likely to be skewed and fat

2 Credit Risk Survey, Risk (1999). 
Chapter 4: Measuring credit spread risk: Incorporating the tails

tailed. This results in the expected loss distribution for credit portfolios being highly skewed and severely fat tailed. Among others, Subrahmanyam, Ho Eom, and Uno (1998) show this for Japanese yen swap spreads and Phoa (1999) provides evidence using Australian dollar swap spread data. In both papers it is argued that incorporating the apparent fat tails is crucial in order to correctly measure credit risk.

Phoa applies Extreme Value Theory (EVT) to parameterise fat tailed FrÈchet, Weibull, and Gumbel distributions in order to measure the maximum expected daily widening in swap spreads on the Australian dollar. The advantage of EVT is that it focuses on the tails and therefore it is suited to be used in a quantile based Value at Risk environment. However, the method used by Phoa to assess the amount of tail fatness (the tail index) is known to be biased. Phoa deals with this fact by showing results for two different tail index estimates. Recent developments in EVT have lead to the development of an unbiased tail index estimator, which has proved to work successfully in measuring the tail index, and therefore is also able to capture the additional downside risk in Value at Risk estimates for stocks and exchange rates (see Huisman, Koedijk, Kool, and Palm (2000), Pownall and Koedijk (1999), and Huisman, Koedijk, and Pownall (1998)).

In this chapter we apply the technique mentioned above to model the tails of the distribution of expected changes in swap spread. Using data from US, UK, German, and Japanese 10 year swap and government bond rates, we provide evidence of the apparent tail fatness in the empirical distributions of the changes. Furthermore, it is shown that the approach outperforms the normal distribution in measuring the risk faced by large widenings or tightenings of credit spreads. 
The plan of the paper is as follows. Section 4.1 focuses briefly on credit spreads. In section 4.2, we discuss the data and provide sample statistics. Section 4.3 introduces tail index estimation and presents the results. Section 4.4 concludes.

\section{1 CREDIT SPREADS}

Defining $\lambda$ as the probability of default, and $\mathrm{f}$ as a fraction representing the recovery rate. The price of a risky bond, $\mathrm{P}^{*}$, can be written as equal to the price of a risk free asset, $\mathrm{P}$ multiplied by the probability of no default over the time interval $t$, plus the value recovered if default does occur ${ }^{25}$.

$$
P_{t}^{*}=(1-(\lambda \Delta t)) \times P_{t}(+\lambda) \Delta t \times f P_{t}
$$

The price of the risky bond can be rewritten as the following equation (4.2),

$$
P_{t}^{*}=P_{t}-(1-f)(\lambda \Delta) P_{t}
$$

So that the expected credit loss, which is measured by the drop in the value due to the possibility of default can be expressed simply as equation (4.3).

2 We follow the notation as in Jorion (1997). This notation implies that the premium for the risky bond is due to the risk of default alone and not to market risk and hence requires no assessment to be made for the size of the market risk premium. 
Chapter 4: Measuring credit spread risk: Incorporating the tails

$$
E C L=(1-f)(\lambda \Delta) P_{t}
$$

The expected credit loss is the probability of default multiplied by the proportion of the position not recovered. So that the credit spread for a given maturity may be written as equation (4.4), where $\lambda$ is the probability of default over the same period as the maturity of the risky and risk free bonds,

$$
y^{*}-y=(1-f) \lambda
$$

The credit spread therefore represents the probability of default multiplied by the proportion not recovered. Indeed using equation (4.4) the term structure of default probabilities can be inferred from the term structures of risky and risk free bonds, in a similar manner to Jarrow and Turnball $(1995)^{26}$. Increasing the number of time intervals, $\mathrm{n}$ to infinity means that the probability of no default $(1-\lambda t)^{n}$ tends to $e^{-\lambda t}$, the price of the risk free asset $\mathrm{Pt}$ tends to $\mathrm{e}^{-\mathrm{yt}}$, and the price of the risky bond, $\mathrm{P} * \mathrm{t}$ tends to $\mathrm{e}^{-\mathrm{y}^{* t}}$. This means we can express the price of the risky bond in equation (4.2) in terms of yields.

$$
e^{-y^{*} \tau}=e^{-\hat{t}} e^{-y \tau}+f\left(1-e^{-\hat{t}}\right) e^{-y \tau}
$$

\footnotetext{
26 Jarrow and Turnball (1995) provide a consistent methodology for pricing and hedging derivative securities involving credit risk, assuming no arbitrage and complete markets.
} 
In a similar manner the credit spread is again a function of the default probability, the recovery rate and now also the time interval. It therefore becomes apparent that from the credit spread we can determine much of the risk involved in credit risk. Indeed it is this factor which is used as the crucial element in credit risk management. For example for the next periods estimate of the expected credit loss we can substitute in the credit spread, the marketsí expectation of default and recovery, as the credit spread. When multiplied by the credit exposure (average price is at the 50\% confidence level), we have an estimate for the expected credit loss, similar to that given in equation (4.2), however now in terms of the credit spread.

$$
E C L=\left(y^{*}-\jmath\right) P^{0.50} t
$$

If however an estimate of the unexpected credit loss is required we multiply the price of the risk free asset instead by the worst credit exposure at a chosen confidence interval, c. For risky debt the credit exposure is the principal, so $\mathrm{P}_{t}^{\mathrm{c}}$ simplifies to the assetsí Value-atRisk for a given confidence level. For products like derivatives it is only when the derivative contract is in the money that potential credit risk arises, so we also need to multiply by the probability of being in the money at time $\mathrm{t}$, denoted by probability $\mathrm{m}$.

$$
U C L=m\left(y^{*}-\right) P^{c}
$$

This approach to estimating unexpected credit loss however does not take into account the risk associated with changes in the size of the credit spread, credit spread risk, or changes 
Chapter 4: Measuring credit spread risk: Incorporating the tails

in the probability of default and the recovery rate. So unless this is incorporated into the worst case Credit-at-Risk $(\mathrm{CaR})$ estimate $^{27}$ it is vital that scenario analysis is used to track the sensitivity of the CaR measure to either credit spread risk or changes in default and recovery rates.

Changes in the credit spread, (credit spread risk) is therefore the risk involved with changes in the size of the credit spread. This can have implications for worst case scenario analysis of credit risk for fixed income products, as well as for pricing credit derivative products where the credit spread is a determining factor for the value of the derivative.

TABLE 4.1 OVERVIEW OF CREDIT RISK DERIVATIVES

\begin{tabular}{|c|c|c|c|}
\hline & $\begin{array}{l}\text { SPREAD } \\
\text { RISK }\end{array}$ & $\begin{array}{l}\text { DEFAULT } \\
\text { RISK }\end{array}$ & $\begin{array}{c}\text { BASKET } \\
\text { INSTRUMENTS }\end{array}$ \\
\hline NOTES & & $\begin{array}{c}\text { CREDIT LINKED } \\
\text { NOTES }\end{array}$ & $\begin{array}{c}\text { CREDIT LINKED } \\
\text { NOTES }\end{array}$ \\
\hline FORWARDS & $\begin{array}{c}\text { CREDIT } \\
\text { FORWARDS }\end{array}$ & & \\
\hline SWAPS & $\begin{array}{c}\text { CREDIT SPREAD } \\
\text { SWAP \& TOTAL } \\
\text { RETURN SWAPS }\end{array}$ & $\begin{array}{c}\text { CREDIT DEFAULT } \\
\text { SWAPS }\end{array}$ & $\begin{array}{c}\text { BASKET CREDIT } \\
\text { SWAPS }\end{array}$ \\
\hline OPTIONS & $\begin{array}{c}\text { CREDIT SPREAD } \\
\text { OPTION }\end{array}$ & $\begin{array}{c}\text { CREDIT DEFAULT } \\
\text { OPTION }\end{array}$ & BASKET OPTIONS \\
\hline
\end{tabular}

27 Even though a confidence level (commonly 95\%) is taken for the distribution of the underlying asset, it is not commonly assumed for the distribution of shifts in the credit spread, however it is a simple exercise to incorporate this directly into the estimate using a bivariate distribution. 
In Table 4.1 we have outlined the various types of credit risk derivatives, and those derivatives whose value depends crucially on credit spread risk, default risk or on a basket products.

In the following section we provide empirical evidence of the probability distribution of credit spread changes, so that sensitivity analysis used in worst case-scenario analysis for credit risk management, and in the pricing and hedging of derivatives products on credit spreads can be more accurately determined.

\section{2 HISTORICAL CREDIT SPREAD WIDENINGS}

To estimate the distribution of shifts in credit spreads for a variety of countries, we employ daily data for the US, UK, Germany and Japan from Datastream over the period January 1990 until January 2000. The credit spread prices the additional risk over a base asset such as the Treasury bill rate. We therefore use 10 year Government Bond yields for the respective countries as the base asset. The swap rate is commonly taken as a proxy for the AA credit rate, since the swap market is significantly deeper and more liquid than that for corporate bonds ${ }^{28}$. We therefore also use the 10 year Datastream Swap Rate, which is a value-weighted index of the middle yield on U.S. swaps. The swap spread (credit spread) is the swap rate less the yield on the current 10-year Government Bond.

28 The Datastream Value-weighted index of the middle yield on U.S. corporate bonds index for example with which includes all maturities and investment grade credit ratings could have been used, however the market is much less liquid with only weekly data available for the same sample period. 
Chapter 4: Measuring credit spread risk: Incorporating the tails

The summary statistics for the daily shifts in credit spreads are given in Table 4. 2. We can see that the average daily shift is extremely small with standard deviations ranging from $6.2 \%$ for Germany, to $8.8 \%$ for the UK. The distribution of credit spread shifts in Japan is highly skewed, and all countries credit markets exhibit significant excess kurtosis.

\section{TABLE 4. 2 SUMMARY STATISTICS}

The table gives the summary statistics for daily shifts in credit spreads over the period January 1990 ñ January 2000 .

\begin{tabular}{l|cccc}
\hline Credit Spread & US & UK & GERMANY & JAPAN \\
\hline Observations & 2610 & 2610 & 2610 & 2610 \\
Average daily shift & $-5.747 \mathrm{E}-06$ & $-2.797 \mathrm{E}-04$ & $1.226 \mathrm{E}-04$ & $-1.782 \mathrm{E}-04$ \\
Standard Deviation & 0.076 & 0.088 & 0.062 & 0.069 \\
Skewness & -0.052 & 0.015 & -0.042 & -0.344 \\
Kurtosis & 7.224 & 9.352 & 7.353 & 28.723 \\
\hline
\end{tabular}

Deviations from normality will result in the probability of large movements in credit spreads being higher than stipulated under the assumption of normally distributed returns. The assumption of gaussian innovations generates a smaller probability of extreme movements, so the assumption of normality is likely to underestimate the credit spread risk of either large tightenings or widenings in credit spreads. The degree of misspecification is 
Chapter 4: Measuring credit spread risk: Incorporating the tails

of course vital for accurate estimation in risk management for both credit risk, and worstcase scenario analysis. The histogram of shifts in swap spreads is given for the US in Figure 4.1 against the probabilities assuming normality. We do indeed observe a greater than normal probability of extreme movements in credit spreads, exemplifying the small but looming potential for increases in default risk to have severe implications on the size of credit spread risk.

\section{FIGURE 4. 1}

\section{HISTOGRAM OF DAILY SPREAD SHIFTS}

This figure gives the quantile estimates for daily shifts in swap spreads over the period January 1990 ñ January 2000. We compare the empirical distribution to that under the assumption of normality.

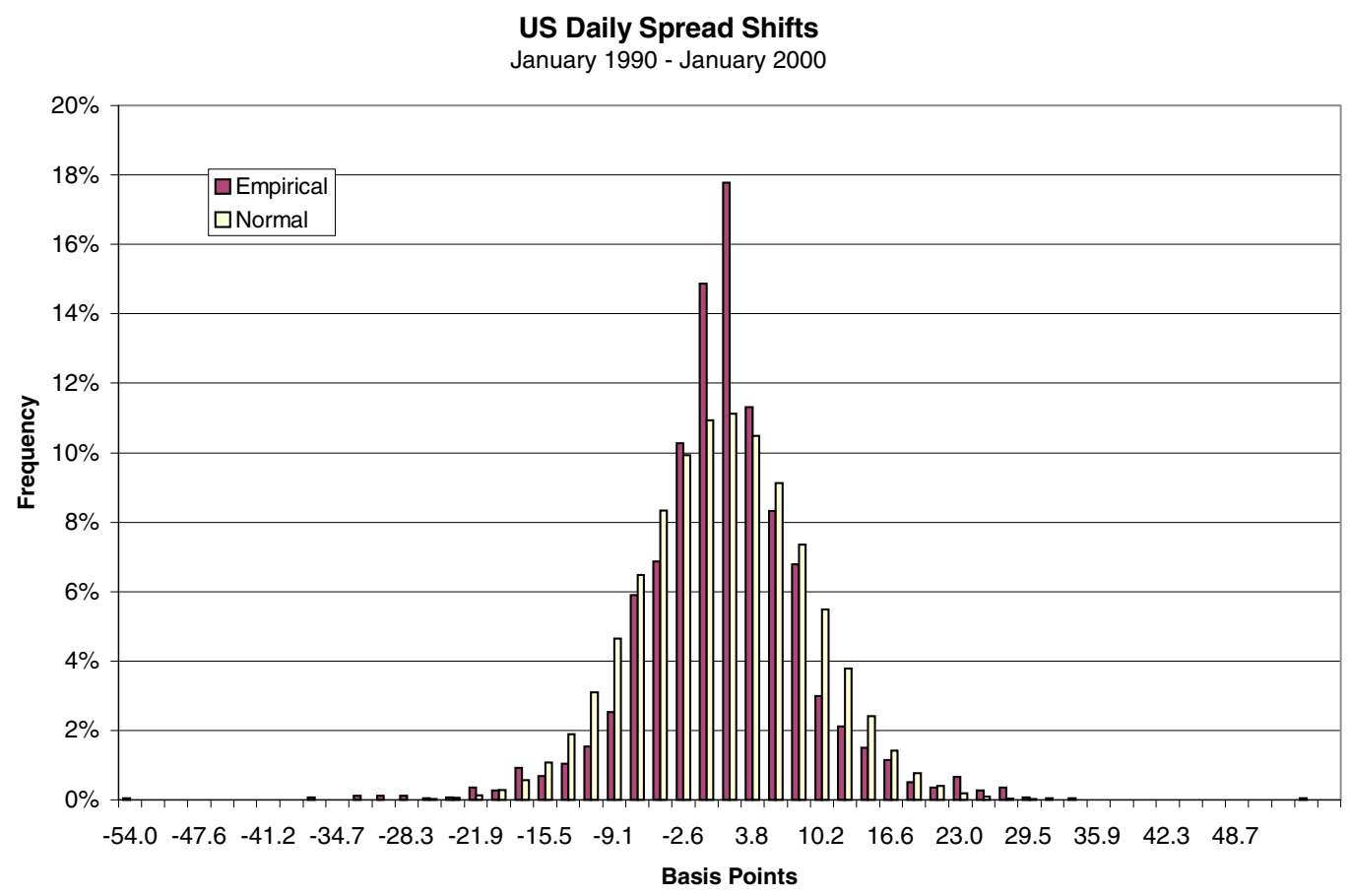


Chapter 4: Measuring credit spread risk: Incorporating the tails

Of course the prevalence of skewed distributions could also result in an alternative probability for larger downward shifts in the swap spread than for upward shifts; so we shall look at both tails of the distribution of shifts in swap spreads. A simple approach to modeling the additional tail fatness in distributions is by parameterising the student-t distribution with degrees of freedom in accordance with the tail estimate of section 2 . This approach follows the approach of Huisman, Koedijk and Pownall (1998) in their VaR-x approach; however instead of focussing directly on Value-at-Risk estimation, here we focus on quantile estimates. These quantile estimates can then be directly incorporated into scenario analysis for Credit-at-Risk analysis, or indirectly, when pricing far out-of-themoney credit risk derivatives.

\section{3 TAIL INDEX ESTIMATION}

Recent developments in the application of Extreme Value Theory to risk management enable us to provide a good estimate of the tail index of the distribution of daily movements in credit spreads. Tail index estimation is the specification of the degree with which the tail of a distribution exhibits tail fatness, and was first introduced by Hill (1975). The tail index measures the speed with which the distributionís tail approaches zero; the fatter the tail the slower the speed and the lower the tail index given. The tail index has the attractive feature that it is equal to the number of existing moments of the distribution, and thus can be used to parameterise the student-t distribution. Hence the link to the fatter tailed Student-t distribution, which nests the normal distribution as a limiting case. We use 
a modified version of the Hill estimator, developed by Huisman et al (1997) to estimate the tail index, which has been modified to account for the bias in the Hill estimator. Specifying $\mathrm{k}$ as the number of tail observations, and ordering their absolute values as an increasing function of size, we obtain the tail estimator proposed by Hill. This is denoted below by $\gamma$ and is the inverse of $\alpha,(4.8)$.

$$
\gamma(k)=\frac{1}{k} \sum_{j=1}^{i} \ln \left(x_{n-j+1}\right)-\ln \left(x_{n-i}\right)
$$

As pointed out by Phoa (1999) in practical applications of the Hill estimator an uncomfortable trade-off exists between variance and bias. This occurs through the use of fewer observations as we move further out into the tails of the distribution, so that although the estimate is less biased (reflects more fully the tail of the distribution) the variance of the estimate increases. The bias of the Hill estimator is therefore a function of the sample size used for the estimate, and is shown in Figure 4.2 for the US swap spread data ${ }^{29}$.

2 A similar pattern emerges for all the series studied. It is interesting to note that the bias is extremely regular when only a very small sample size is used. This is likely to be driven by the low but equal frequency of extremely large credit widenings and tightenings. 
Chapter 4: Measuring credit spread risk: Incorporating the tails

\section{FIGURE 4. 2 HILL TAIL INDEX ESTIMATOR}

This figure gives the bias in the Hill estimator as the sample size $m$ increases for the tail index estimation as given in equation (4.7). Daily shifts in US swap spreads over the period January 1990 ñ January 2000.

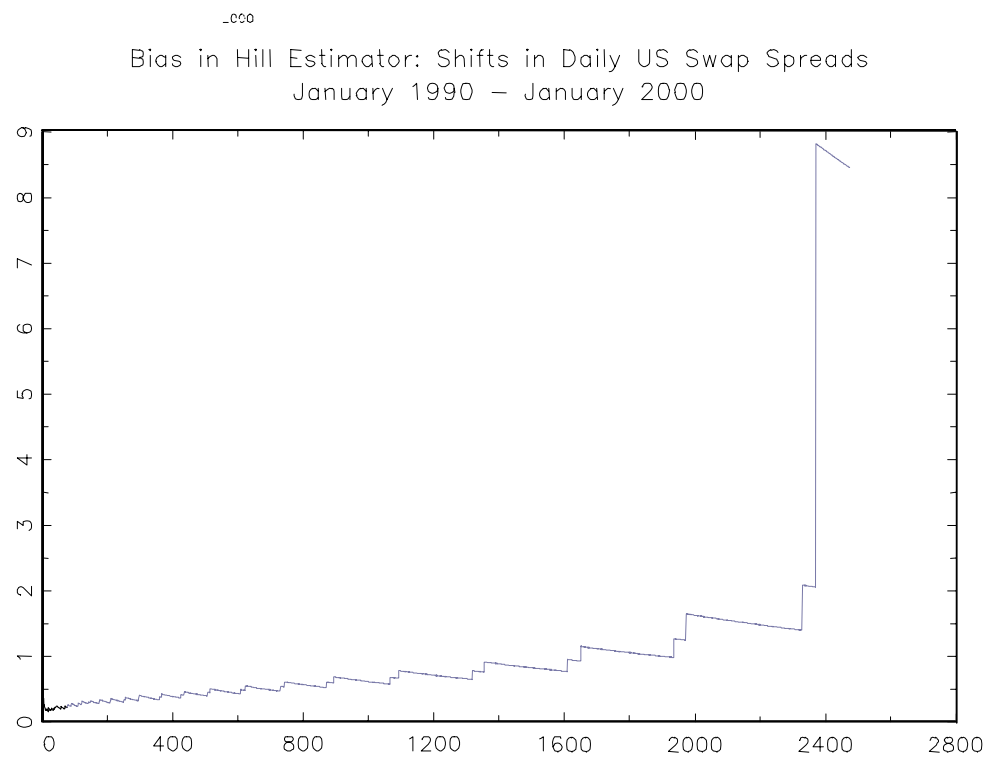

Following the methodology of Huisman et al. (1997), we can use a modified version of the Hill estimator (1997) to correct for the bias in small samples. A bias corrected tail index is therefore obtained by observing the bias of the Hill estimator as the number of tail observations increases up until $\kappa$, whereby $\kappa$ is equal to half of the sample size:

$$
\gamma(k)=\beta_{0}+\beta_{1} k+\varepsilon(k), k=1 \ldots . \kappa
$$

The optimal estimate for the tail index is the intercept $\beta_{0}$. And the $\alpha$ estimate is just the 
Chapter 4: Measuring credit spread risk: Incorporating the tails

inverse of this estimate. This is the estimate of the tail index that we use to parameterise the Student-t distribution. Recent applications of this approach for estimating market risk have been shown to work well for a variety of financial time series ${ }^{30}$. We have estimated the tail estimates using the alpha HKKP estimator for the four countries, and the estimates for both tails, the left tail and the right tail respectively are given in Table 4. 3 .

\section{TABLE 4. 3 ALPHA ESTIMATES}

The table gives the alpha estimates for daily shifts in credit spreads over the period January 1990 - January 2000, using the HKKP estimator.

\begin{tabular}{l|cccc}
\hline & US & UK & GERMANY & JAPAN \\
\hline ALPHA (BOTH) & $\mathbf{3 . 8 4 8}$ & $\mathbf{3 . 4 2 3}$ & $\mathbf{3 . 5 5 0}$ & $\mathbf{2 . 9 3 9}$ \\
KAPPA & 1305 & 1305 & 1305 & 1305 \\
ALPHA (LEFT) & $\mathbf{3 . 9 5 7}$ & $\mathbf{3 . 0 3 5}$ & $\mathbf{2 . 8 0 3}$ & $\mathbf{2 . 7 3 5}$ \\
KAPPA & 603 & 618 & 732 & 582 \\
ALPHA (RIGHT) & $\mathbf{4 . 5 0 6}$ & $\mathbf{3 . 8 3 5}$ & $\mathbf{4 . 2 3 0}$ & $\mathbf{3 . 5 6 1}$ \\
KAPPA & 701 & 686 & 572 & $\mathbf{7 2 3}$ \\
\hline
\end{tabular}

Since all the series exhibited excess kurtosis it is not surprising that the alpha estimates used to parameterise the student-t distribution generate much fatter tailed distributions than

30 See Huisman, Koedijk \& Pownall (1998) for an application to US stocks and Bonds, and Pownall \& Koedijk (1999) for Asian stock markets, as well as Campbell, Eicholtz \& Huisman (2000) for the US and Dutch real estate markets. 
Chapter 4: Measuring credit spread risk: Incorporating the tails

under normality. We also observe that the alpha estimate for the left tail alone for all the series is slightly smaller than the estimate using both tails, and the right tail of the distribution only. This provides evidence of a greater probability attached to credit spread tightenings than to credit spread widenings. This may result from the fact that sharp rises in Treasury yields appear more frequently than sharp falls ${ }^{31}$. We therefore analyse the quantile estimates for the downward and upward shifts in credit spreads separately, using the tail index estimator for the respective tail.

In Figure 4.3 we have plotted the quantile estimates using the two approaches for quantiles ranging from $7.5 \%$ to $1 \%$ and $92.5 \%$ to $99 \%$ in the right and left tails of the distribution for US daily shifts in credit spreads. Of course it is the large upward shifts in swap spreads, which are the cause for concern, since financial institutions tend to be long, rather than short, in government bonds.

For the more extreme cases the assumption of normality severely underestimates the size of the potential shift in the credit spread shift. Indeed this is the case for all the series which we analysed. The results for the quantile estimates for potential daily tightenings and widenings are given in tables 4.4 and 4.5 for the US, UK, Germany and Japan.

31 See Phoa (1999). 
Chapter 4: Measuring credit spread risk: Incorporating the tails

FIGURE 4. 3 QUANTILE ESTIMATES UNDER ALTERNATIVE PARAMETRIC

\section{DISTRIBUTIONAL ASSUMPTIONS}

This figure gives the quantile estimates for daily shifts in swap spreads over the period January 1990 - January 2000, assuming normality and the student-t distribution, with degrees of freedom parameterised by the HKKP alpha estimates for the left and right tails of the distribution respectively as described in Table 4. 3.

US Downward Swap Shifts

January 1990 - January 2000



percentile

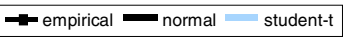

US Upward Swap Shifts

January 1990 - January 2000

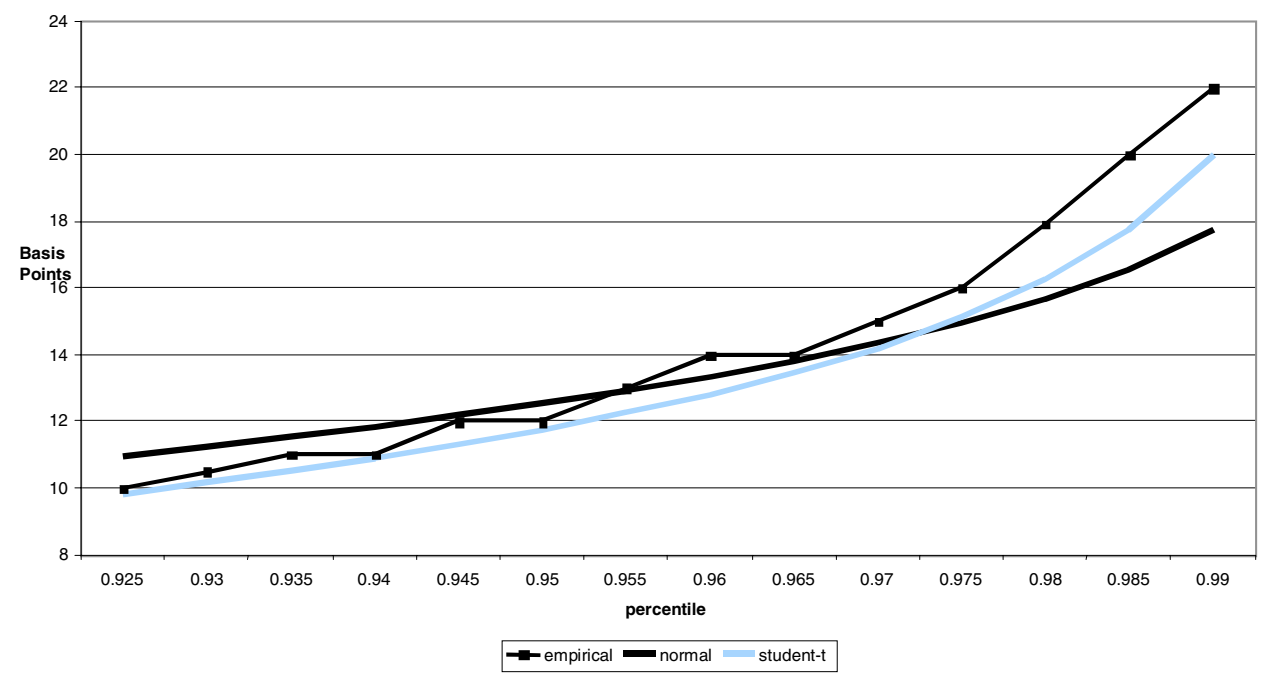


Chapter 4: Measuring credit spread risk: Incorporating the tails

\section{TABLE 4. 4 CREDIT SPREAD TIGHTENINGS ñ QUANTILE ESTIMATES}

The table gives the quantile estimates for daily shifts in swap spreads over the period January 1990 ñ January 2000, assuming normality and the student-t distribution, with degrees of freedom parameterised by the alpha estimates for the left tail as described in Table 4. 3.

\begin{tabular}{|c|c|c|c|}
\hline Monthly Event 4.76\% & EMPIRICAL & NORMAL & STUDENT-T $\left(\alpha_{\mathrm{L}}\right)$ \\
\hline US & -12.0 & -12.7 & -11.7 \\
\hline $\mathbf{U K}$ & -13.0 & -14.7 & -12.3 \\
\hline GERMANY & -9.0 & -10.4 & -8.3 \\
\hline JAPAN & -9.0 & -11.5 & -9.0 \\
\hline Yearly Event $0.397 \%$ & EMPIRICAL & NORMAL & STUDENT-T $\left(\alpha_{\mathrm{L}}\right)$ \\
\hline $\mathbf{U S}$ & -29.6 & -20.2 & -26.6 \\
\hline UK & -35.0 & -23.4 & -32.2 \\
\hline GERMANY & -24.7 & -16.5 & -22.8 \\
\hline JAPAN & -31.9 & -18.3 & -25.1 \\
\hline 5-Yearly Event $0.079 \%$ & EMPIRICAL & NORMAL & STUDENT-T $\left(\alpha_{L}\right)$ \\
\hline $\mathbf{U S}$ & -37.0 & -24.1 & -41.4 \\
\hline UK & -57.4 & -27.8 & -55.8 \\
\hline GERMANY & -32.5 & -19.7 & -41.1 \\
\hline JAPAN & -62.9 & -21.8 & -46.0 \\
\hline 10-Yearly Event $0.040 \%$ & EMPIRICAL & NORMAL & STUDENT-T $\left(\alpha_{\mathrm{L}}\right)$ \\
\hline $\mathbf{U S}$ & -53.4 & -25.6 & -49.7 \\
\hline UK & -58.9 & -29.5 & -70.4 \\
\hline GERMANY & -35.9 & -20.9 & -52.7 \\
\hline JAPAN & -69.7 & -23.1 & -59.4 \\
\hline 20-Yearly Event $0.019 \%$ & EMPIRICAL & NORMAL & STUDENT-T $\left(\alpha_{\mathrm{L}}\right)$ \\
\hline US & - & -27.0 & -59.5 \\
\hline $\mathbf{U K}$ & - & -31.2 & -88.7 \\
\hline GERMANY & - & -22.1 & -67.7 \\
\hline JAPAN & - & -24.4 & -76.7 \\
\hline
\end{tabular}




\section{TABLE 4. 5 CREDIT SPREAD WIDENINGS ñ QUANTILE ESTIMATES}

The table gives the quantile estimates for daily shifts in swap spreads over the period January 1990 ñ January 2000, assuming normality and the student-t distribution, with degrees of freedom parameterised by the alpha estimates for the right tail as described in Table 4. 3.

\begin{tabular}{|c|c|c|c|}
\hline Monthly Event 4.76\% & EMPIRICAL & NORMAL & STUDENT-T $\left(\alpha_{R}\right)$ \\
\hline US & 12.9 & 12.7 & 12.0 \\
\hline $\mathbf{U K}$ & 14.0 & 14.7 & 13.5 \\
\hline GERMANY & 10.0 & 10.4 & 9.7 \\
\hline JAPAN & 10.0 & 11.5 & 10.3 \\
\hline Yearly Event $0.397 \%$ & EMPIRICAL & NORMAL & STUDENT-T $\left(\alpha_{R}\right)$ \\
\hline US & 26.3 & 20.2 & 25.8 \\
\hline UK & 33.5 & 23.4 & 31.0 \\
\hline GERMANY & 23.6 & 16.5 & 21.3 \\
\hline JAPAN & 26.3 & 18.3 & 24.6 \\
\hline 5-Yearly Event $0.079 \%$ & EMPIRICAL & NORMAL & STUDENT-T $\left(\alpha_{R}\right)$ \\
\hline US & 29.9 & 24.1 & 38.5 \\
\hline UK & 43.7 & 27.9 & 48.7 \\
\hline GERMANY & 29.9 & 19.6 & 32.5 \\
\hline JAPAN & 38.4 & 21.8 & 39.8 \\
\hline 10-Yearly Event $0.040 \%$ & EMPIRICAL & NORMAL & STUDENT-T $\left(\alpha_{R}\right)$ \\
\hline US & 31.9 & 25.6 & 45.4 \\
\hline UK & 52.2 & 29.6 & 58.8 \\
\hline GERMANY & 31.0 & 20.9 & 38.7 \\
\hline JAPAN & 40.4 & 23.2 & 48.7 \\
\hline 20-Yearly Event $0.019 \%$ & EMPIRICAL & NORMAL & STUDENT-T $\left(\alpha_{R}\right)$ \\
\hline US & - & 27.0 & 53.3 \\
\hline $\mathbf{U K}$ & - & 31.2 & 70.7 \\
\hline GERMANY & - & 22.0 & 45.8 \\
\hline JAPAN & - & 24.4 & 59.2 \\
\hline
\end{tabular}


The probability of credit spread tightenings have historically been slightly larger than upward movements. However all the results provide evidence of severe underestimation of the potential changes in large movements of credit spreads. Indeed the fatter tailed student$\mathrm{t}$ distribution parameterised by the alpha tail index estimator provides basis point movements for monthly, yearly, 5, and 10 yearly events much more in line with those having been observed in recent years. It would therefore appear to be much more prudent to use these larger estimates in risk management techniques and derivative pricing and hedging strategies incorporating credit spread risk.

\section{4 CONCLUDING REMARKS}

Estimation of credit spread risk is not only important for pricing and hedging credit derivatives but also for accurate risk management. Small but looming possibilities of default however render the expected return distribution for financial products containing credit risk to be non-normal. To correctly assess the true probability of large movements in credit widenings and tightenings we apply recent techniques developed to incorporate additional downside risk resulting from non-normalities in managing market risk to data on swaps and swap spreads. The downside of our results is that for unexpected events the assumption of normality results in credit spread risk in many countriesí credit markets being grossly underestimated. Estimation of swap and credit spread risk for such events is dramatically improved when the severity of the additional downside risk is measured and incorporated into current estimation techniques. These results are not only crucial for improving credit risk management but also in pricing out-of the money credit derivatives. 
Chapter 4: Measuring credit spread risk: Incorporating the tails 
Chapter 5: Times of crisis: A conditional or unconditional approach?

\section{CHAPTER 5 TIMES OF CRISIS: A CONDITIONAL OR UNCONDITIONAL APPROACH? ${ }^{32}$}

Using data on Asian equity markets, we observe that during periods of financial turmoil, deviations from the mean-variance framework become more severe, resulting in periods with additional downside risk to investors. Current risk management techniques failing to take this additional downside risk into account will underestimate the true Value-at-Risk with greater severity during periods of financial turmoil. We provide a conditional approach to the Value-at-Risk methodology, known as conditional VaR-x, which to capture the time variation of non-normalities allows for additional tail fatness in the distribution of expected returns. These conditional VaR-x estimates are then compared with those based on the RiskMetrics ${ }^{T M}$ methodology from J.P. Morgan, where we find that the model provides improved forecasts of the Value-at-Risk. We are therefore able to show that our conditional VaR-x estimates are better able to capture the nature of downside risk, particularly crucial in times of financial crises. 
A number of Asian economies have recently been characterized by highly volatile financial markets, which when coupled with high returns, should have been seen as an attractive avenue down which one could diversify portfolios. The recent financial turmoil, however, has had a severe impact on investorsí exposure to these markets, with investors having fled these markets en masse ${ }^{33}$. The risk thus appears to have been too great and the resultant capital flight has led to even greater pressures, creating further turbulence in markets all over Asia.

To ensure a stabile financial system, being able to accurately identify, measure, and control financial risk are $\operatorname{crucial}^{34}$. This is why regulation was introduced worldwide. The goal has been to tighten up risk management, and thus avoid the potential damage from bank runs and systemic risk. These regulatory changes in turn have had two dimensions: the imposition of minimum capital requirements for financial institutions as designed by the Basle Committee ${ }^{35}$ and the adoption of the Value-at-Risk method of assessing capital adequacy as a risk management technique. Banks are now required to hold enough capital to be able to withstand large potential losses. With recurrent banking crises occurring all over Asia it would appear that the risk during such periods was of a much greater magnitude than existing risk management techniques were able to capture.

\footnotetext{
This chapter is based on R. A. Pownall and K.G. Koedijk, 'Capturing Downside Risk in Financial Markets: The Case of the Asian Crisis' Journal of International Money and Finance, (1999).

33 See the Economist (1998)

34 Jorion (1997) gives a good overview of Value-at-Risk.

35 Basle Committee on Banking Supervision (1996).
} 


\section{Chapter 5: Times of crisis: A conditional or unconditional approach?}

Large negative returns on Emerging market returns have been shown to occur more frequently than predicted under the assumption of normality, even when the distribution is made conditional ${ }^{36}$. The use of the estimated variance of an assetís return distribution as the sole measure of risk may therefore lead to a serious under-estimation of the true risk involved in holding such assets. We investigate the implications of such non-normality for risk management in general and the estimation of Value-at-Risk in particular. We show that the use of an additional parameter to account for the additional downside risk can provide a more accurate tool for risk management. This is the conditional VaR-x $\operatorname{method}^{37}$.

In periods of turmoil in which the risk is higher, we find the deviations from conditional normality to be even greater, and in periods of financial crisis they become very severe indeed. This additional downside risk is not captured in current VaR methods, which assume conditional-normality, such as in the J.P. Morgan RiskMetricsô methodology.

The implications from the inclusion of additional downside risk however go beyond the use of Value-at-Risk as a risk management technique. If volatility alone is insufficient in estimating the amount of risk, then the additional use of downside risk may provide us with a more accurate measure for risk. Pricing risk lies at the heart of finance theory and an improved measure for risk may unravel many of the puzzles concerning the size of the premium attached to risk. For example the equity premium puzzle. Using volatility to

\footnotetext{
36 See Bekaert, Erb, Harvey and Viskanta (1998) for an analysis of the time-varying nature of the distributional characteristics of Emerging Market returns.

37 See Huisman, Koedijk and Pownall (1998) for an application of unconditional VaR-x.
} 
measure risk, and a moderate level for investorsí aversion to risk, the premium paid for taking on the additional risk from investing in equity is excessively large. Additional risk in the downside implies a greater level of risk for a given level of return, and hence the premium attached would be more in line with observed attitudes to risk. Another puzzle is the tendency for investors to invest a greater proportion of their assets in the domestic market than finance theory would suggest, the so-called home bias phenomenon. Again given a moderate level for investorsí risk aversion, and volatility as the measure for risk, a similar puzzle to the equity premium puzzle arises: the risk of investing internationally appears to be too great for the premium received (home bias). Including the additional downside risk into the measure for risk may therefore explain the extent of the risk perceived by investors, and for the premium prevailing, why investors tend to prefer to invest in their home markets.

The chapter is organized as follows. We begin in section 5.1 by introducing the current risk management technique of Value-at Risk, and discuss its suitability for the Asian markets. In section 5.2 we analyze downside risk, particularly apparent in periods of financial crises. Estimating Value-at Risk conditionally and unconditionally is introduced in section 5.3. Here we compare current approaches, emphasizing in particular their ability to forecast Value-at-Risk during periods of financial crises. The implications from the inclusion of downside risk for asset allocation is pursued in section 5.4. Conclusions are drawn in the final section of the paper. 


\subsection{RISK MANAGEMENT IN ASIAN FINANCIAL MARKETS}

The accurate measurement and control of financial risk in international markets is crucial to institutions exposed internationally. Asian markets have been characterised as particularly risky over recent years, with extremely volatile returns on equity markets. Looking at the IFC Asia $50 \operatorname{Index}^{38}$ on a daily basis, for the whole period for which data is available, January 1993 until January 1998, we see that average volatility on a yearly basis has been $22.04 \%$.

By the end of 1997 , the average yearly return since 1993 has been negative, $\tilde{\text { ñ }} .18 \%$; with the enormous growth of the early nineties having been more than completely wiped out during the financial crises in 1997 alone. Summary statistics for the entire period for which data are available along with the individual years is given in Table 5. 1. Taking the period as a whole the data also exhibit highly significant skewness and kurtosis.

When breaking the sample down into years, with an average of 259 trading days each, we see that the unprecedented $92.68 \%$ drop in the value of equities during the financial crises in 1997 was accompanied by a higher than average volatility at $31.29 \%$. Indeed the only other year in the sample with a negative return, be it a much less significant fall of $\tilde{n}$ $14.58 \%$ in 1994 , was also a year of higher than average volatility.

38 IFC emerging market indices are employed by Bekaert and Campbell (1995) where they discuss the suitability of the data series for empirical work. 
It is this measure, the standard deviation of the return distribution, which is most commonly used in financial theory for capturing risk: implying that the more frequent the occurrence of large returns, whether positive or negative, the higher the expected exposure to risk. The higher the volatility the greater the risk faced by institutions. Risk management techniques, such as Value-at-Risk, aim to capture the increased market risk concurrent with more volatile financial assets, and since Value-at-Risk is associated with a particular fractile of the distribution, the use of normality also pertains the use of the standard deviation as the measure for risk.

\subsubsection{UNCONDITIONAL VALUE-AT-RISK}

The most commonly used technique in risk management to assess possible losses in financial markets is Value-at-Risk. By estimating the worst expected loss over a chosen time horizon, within a given confidence interval, it aims to summarise the market risk. If an amount $\mathrm{W}_{0}$ is the initial portfolio investment, then taking $\mathrm{R}$ as the rate of return, the expected value of the portfolio at the end of a chosen time horizon is:

$$
\mathrm{W}=\mathrm{W}_{0}(1+\mathrm{R})
$$




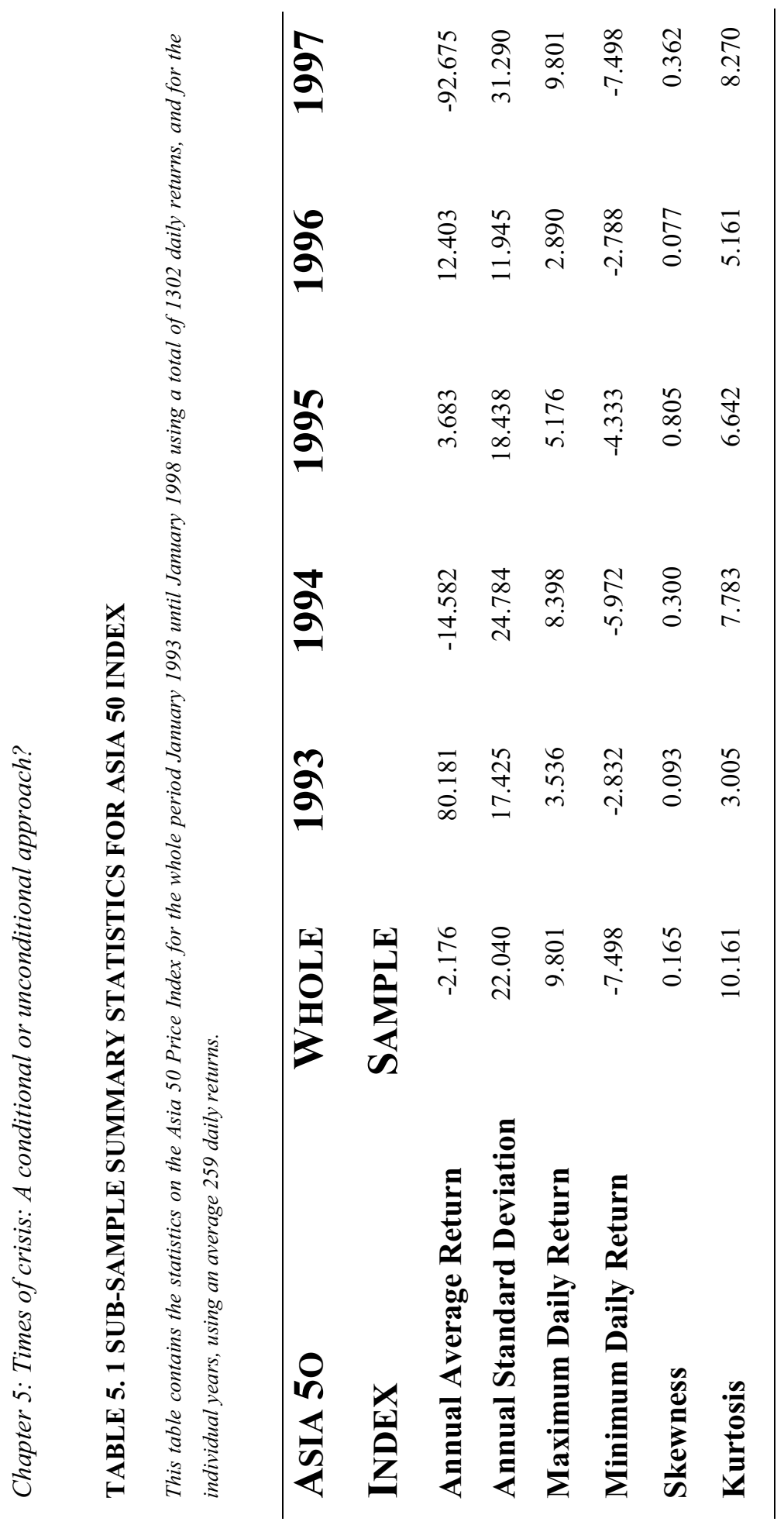


Chapter 5: Times of crisis: A conditional or unconditional approach?

Since we are interested in the lowest portfolio value at a particular confidence level, denoted by $\mathrm{c}$, we are interested in finding the rate of return $\mathrm{R}^{*}$ resulting in this lowest portfolio value $\mathrm{W}^{*}$ :

$$
\mathrm{W}^{*}=\mathrm{W}_{0}\left(1+\mathrm{R}^{*}\right) .
$$

Letting the average return be denoted by $\mu$, gives us the estimate for the VaR relative to the mean to be written as:

$$
\mathrm{VaR}=\mathrm{W}_{0}(1+\mu)-\mathrm{W}_{0}\left(1+\mathrm{R}^{*}\right)
$$

Simplifying to:

$$
\mathrm{VaR}=-\mathrm{W}_{0}\left(\mathrm{R}^{*}-\mu\right)
$$

The crux of being able to provide an accurate estimate for the Value-at-Risk is in being able to accurately estimate the expected return $\mathrm{R}^{*}$ associated with the portfolio value $\mathrm{W}^{*}$. Value-at-Risk estimation therefore requires knowing the probability distribution of the expected returns, which of course is unknown. Hence the various methods for estimating VaR depend on the assumption made about the probability distribution of the expected returns. 


\section{Chapter 5: Times of crisis: A conditional or unconditional approach?}

One method is to consider the actual empirical distribution, based on past observations, as best representing the probability distribution of expected returns. The historical VaR is then found from substituting the point $\mathrm{R}^{*}$ from the histogram of the empirical distribution based on historical returns into the above formula. $\mathrm{R}^{*}$ is the point, below which, the fraction 1-c of the returns fall.

Alternatively one can assume that the returns can be approximated by a specific statistical distribution, with the exact form of the analytical distribution being determined from parameters, estimated from past observations. For example, it has been commonly assumed in finance theory that asset returns are normally distributed. Then the point on the standard normal distribution $\mathrm{N}^{*}$, at which the area 1-c falls to the left, can be converted to a distribution with mean $\mu$, and standard deviation $\sigma$, to find the cut off return $\mathrm{R}^{*}$ :

$$
\mathrm{R}^{*}=\tilde{\mathrm{n}} \mathrm{N}^{*} \sigma+\mu
$$

Substituting this value for $\mathrm{R}^{*}$ into equation (5.4) gives us the relative parametric-normal VaR equal to $\mathrm{W}_{0} \mathrm{~N}^{*} \sigma$. Hence by assuming normally distributed returns only the standard deviate of the portfolio, multiplied by a factor depending on the confidence level, is required to find the relative $\mathrm{VaR}$.

The estimates of the VaR using the two approaches for various confidence levels are presented for the whole period in the first two columns of Table 5. 2. We see how the VaR estimates for both estimates increase, the higher the confidence level taken. 
Chapter 5: Times of crisis: A conditional or unconditional approach?

\section{TABLE 5. 2 COMPARISON OF VALUE-AT-RISK ESTIMATES}

The Value-at-Risk Estimates have been estimated for the two asset classes using the Empirical Approach (using the historical data) and the Parametric-Normal approach. The Normal VaR estimates assume Normally distributed returns, The relative VaR estimates, expressed in millions of dollars, have been calculated for a position of $\$ 100$ million in the particular asset, and for a range of confidence levels.

\begin{tabular}{|c|c|c|c|c|c|c|}
\hline & \multicolumn{6}{|c|}{$\begin{array}{l}\text { ASIA 50 } \\
\text { INDEX }\end{array}$} \\
\hline $\begin{array}{l}\text { Confidence } \\
\text { Level } \\
\text { (Left Tail) }\end{array}$ & $\begin{array}{r}\text { Empirical } \\
\mathbf{V a R} \\
(\$ 100 \\
\text { mill. })\end{array}$ & $\begin{array}{r}\text { Normal } \\
\text { VaR } \\
(\$ 100 \\
\text { mill. })\end{array}$ & $\begin{array}{r}\text { Empirical } \\
\mathbf{V a R} \\
(\$ 100 \\
\text { mill. })\end{array}$ & $\begin{array}{r}\text { Normal } \\
\text { VaR } \\
(\$ 100 \\
\text { mill. })\end{array}$ & $\begin{array}{r}\text { Empirical } \\
\text { VaR } \\
(\$ 100 \\
\text { mill. })\end{array}$ & $\begin{array}{r}\text { Normal } \\
\text { VaR } \\
(\$ 100 \\
\text { mill. })\end{array}$ \\
\hline 95 & 1.9896 & 2.2484 & 1.1962 & 1.2191 & 3.1538 & 3.1814 \\
\hline 95.5 & 2.1398 & 2.3175 & 1.2103 & 1.2566 & 3.3067 & 3.2792 \\
\hline 96 & 2.2372 & 2.3931 & 1.2403 & 1.2976 & 3.4081 & 3.3861 \\
\hline 96.5 & 2.3736 & 2.4768 & 1.2565 & 1.343 & 3.5589 & 3.5046 \\
\hline 97 & 2.4869 & 2.5709 & 1.3181 & 1.394 & 3.724 & 3.6378 \\
\hline 97.5 & 2.8156 & 2.6792 & 1.3245 & 1.4527 & 4.0313 & 3.7909 \\
\hline 98 & 3.1603 & 2.8074 & 1.5347 & 1.5222 & 4.6725 & 3.9723 \\
\hline 98.5 & 3.5866 & 2.9664 & 2.2766 & 1.6084 & 5.607 & 4.1974 \\
\hline 99 & 4.2016 & 3.18 & 2.3117 & 1.7243 & 6.4717 & 4.4996 \\
\hline
\end{tabular}


Chapter 5: Times of crisis: A conditional or unconditional approach?

\section{FIGURE 5. 1 VALUE-AT-RISK ESTIMATES}

The graph depicts how much the Parametric-Normal VaR estimates differ from the Empirical VaR estimates for the two sub-samples of data from the Asia 50 Index over a range of confidence levels. The Parametric-Normal approach assumes Normally distributed returns and the Empirical Approach uses the observed frequency distribution. The difference is the error generated by using the assumption of Normally distributed returns and is estimated for a $\$ 100$ million position in the particular asset.

\section{Deviations from from Daily Value-at-Risk in Million Dollars: Parametric-Normal Value-at-Risk}

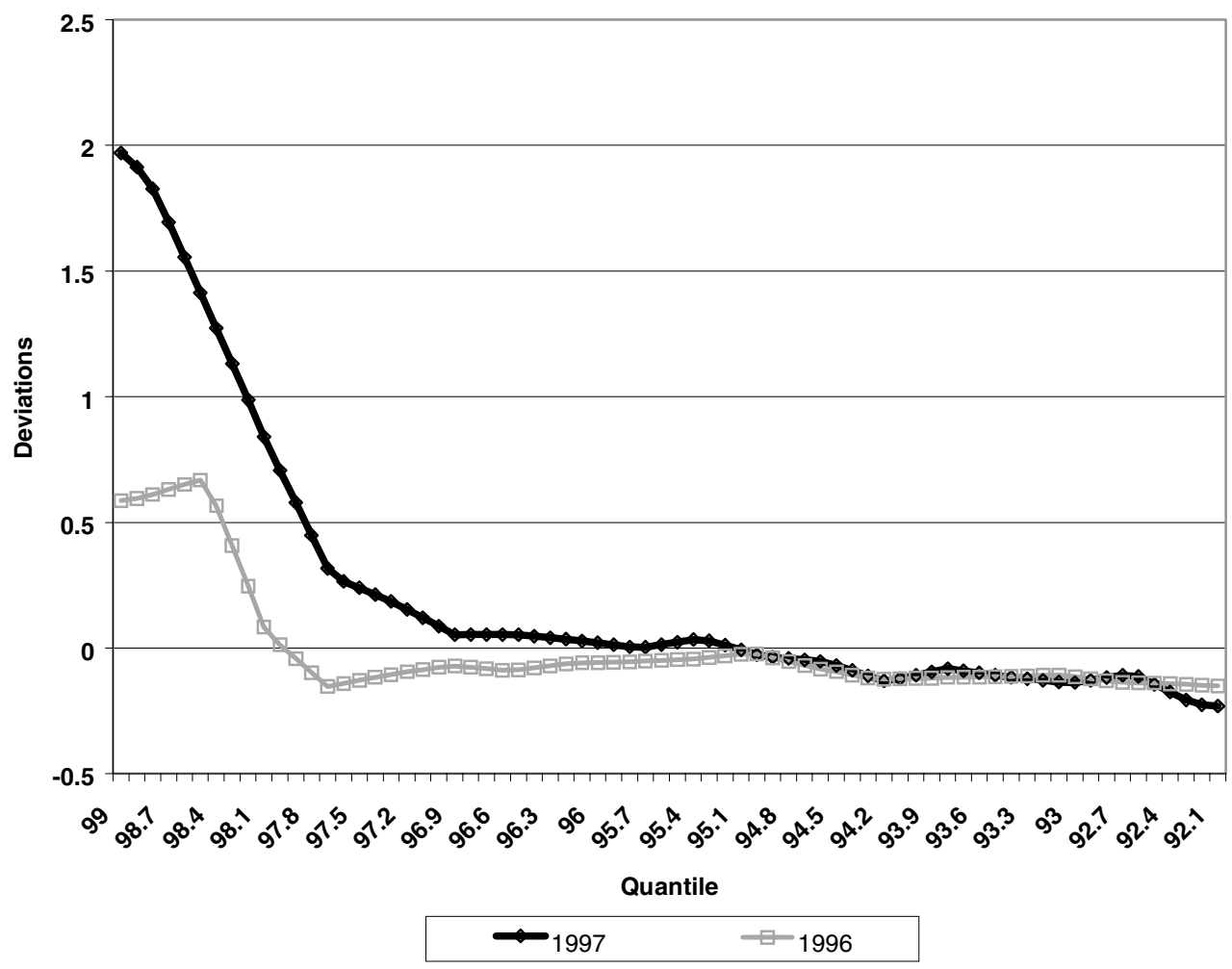


Chapter 5: Times of crisis: A conditional or unconditional approach?

\section{FIGURE 5. 2 VALUE-AT-RISK ESTIMATES}

The graph depicts how the inverse alpha estimates for the tail index vary over time. The tail index is estimated using a modified version of the Hill estimator and uses the previous years data (260 daily observations) for estimation. Also plotted are the actual daily returns on the Asia 50 Index.

A sia 50 D aily Index: D ownside $R$ isk and $R$ etums overtin e

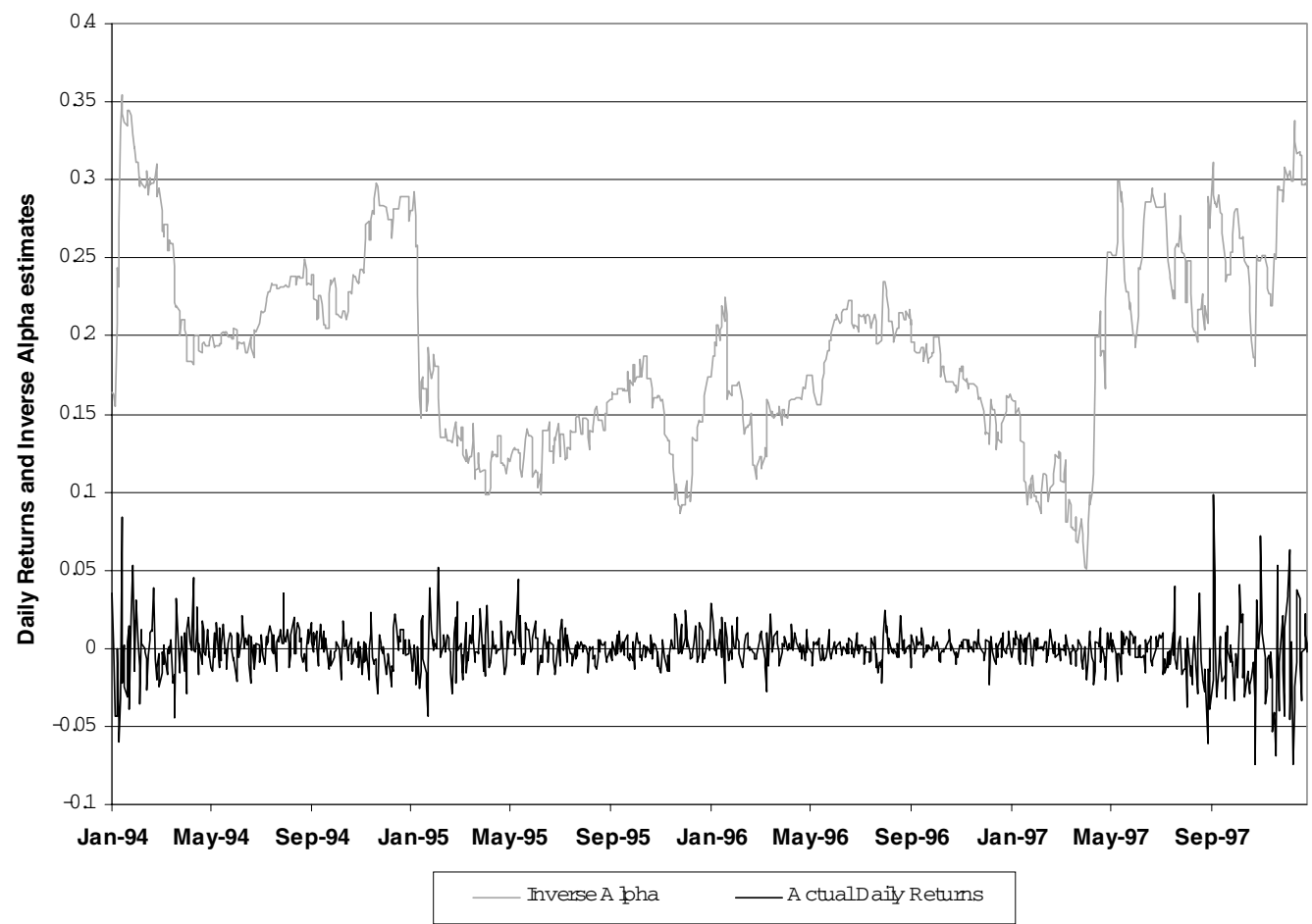




\section{Chapter 5: Times of crisis: A conditional or unconditional approach?}

However the parametric-normal approach underestimates the exposure to market risk at higher confidence levels, with the difference growing as we move further into the tail of the distribution. This is due to the existence of non-normality in the data as a negatively skewed distribution with fatter than normal tails will tend to generate higher VaR estimates than captured by the assumption of normality.

During periods of high volatility however, the VaR estimates are by definition larger, and hence any deviations from normality become more crucial. Indeed we see that the deviations from normality are more severe in 1997, during the financial crises, than in 1996, when the distribution exhibits highly significant skewness and kurtosis. Hence the deviation in the VaR estimate from using the parametric-normal distribution is also greater. The extent to which the parametric-normal underestimates the VaR at high confidence levels during the period of financial crises is depicted in Figure 5.1. The sample period has been split into two, with parameters having been unconditionally estimated for the two periods specifically.

It is well documented that the return distributions of many financial assets show serious deviations from normality ${ }^{39}$, so that the VaR tends to be underestimated as we move to higher confidence intervals. Bekaert et al. (1998) give some well known country characteristics to try to explain the degree with which emerging market data deviate from normality. We indeed find that the distribution is more fat tailed during the financial crises, exhibiting more frequent extreme returns than the normal distribution. Parametric methods

39 See for example Mandelbrot (1963), Fama \& Roll (1965), Taylor (1986) and Huisman et al. (1998). 
Chapter 5: Times of crisis: A conditional or unconditional approach?

for estimating the VaR using the assumption of normality therefore severely underestimate the VaR as we move to higher confidence levels. This is depicted in Figure 5.2.

The extra probability mass should be partially captured in the tails by allowing for the distribution to be time varying. Allowing for a conditional distribution to capture this changing volatility over time can easily be implemented into the VaR estimation. A Generalised Autoregressive Conditionally Heteroskedastic (GARCH) process can be used to estimate conditional volatility, and thus substituted into equation 5. Estimating a GARCH process is the basis of the RiskMetrics ${ }^{\mathrm{TM}}$ methodology introduced by JP Morgan.

\subsubsection{RISKMETRICS ${ }^{\mathrm{TM}}$}

Implementing conditional volatility into equation 5 by means of a GARCH model is the approach adopted in the RiskMetricsís ${ }^{\mathrm{TM}}$ methodology. It is well known that volatility tends to exhibit clustering behaviour, with periods of high and then low volatility. This type of behaviour was first captured by Engle (1982) through the use of an Autoregressive Conditional Heteroskedastic (ARCH) process. ARCH modeling allows the conditional variance to change over time leaving the unconditional variance constant. The $\mathrm{ARCH}$ process was generalised by Bollerslev (1985) so that the conditional variance is not only a function of past errors but also of lagged conditional variances. GARCH modeling has since then become extremely popular in empirical applications for the second moment in financial time series. A GARCH(p,q) process can be written as: 


$$
h_{t}=\alpha_{0}+\sum_{i=1}^{q} \alpha_{i} \varepsilon_{t-i}^{2}+\sum_{i=1}^{p} \beta_{i} h_{t-i}
$$

Where $\varepsilon_{t}^{2}$ is the sample variance, and $h_{t}$ is the conditional variance both at time $t$. Following the RiskMetrics ${ }^{\mathrm{TM}} 40$ methodology, the optimal conditional variance is estimated by a $\operatorname{GARCH}(1,1)$ model with zero constant and the parameters $\alpha$ and $\beta$ summing to unity. Imposing such a restriction gives us a process formally known as Integrated GARCH (IGARCH):

$$
h_{t}=\lambda h_{t-1}+(1-\lambda) \varepsilon^{2}{ }_{t-1}
$$

Instead of estimating volatility unconditionally using an equally weighted moving average, the RiskMetrics ${ }^{\mathrm{TM}}$ approach therefore uses exponential weights, so that the more recent observations weigh more heavily. The rate of decline of the exponential weights depends on the decay factor $\lambda$, thus expressing the persistence with which a shock will decay. They suggest setting the decay factor to 0.94 for daily data and 0.97 when using monthly data. The fact that only the one parameter $\lambda$ need be estimated facilitates estimation and provides for greater robustness against estimation error ${ }^{41}$.

See the J.P. Morgan RiskMetrics Technical Manual (1996) for an introduction to RiskMetrics ${ }^{\mathrm{TM}}$. 41 See Jorion (1997) for a good discussion of the applicability of the RiskMetrics ${ }^{\mathrm{TM}}$ Methodology. 
Chapter 5: Times of crisis: A conditional or unconditional approach?

TABLE 5. 3 NUMBER OF EXCEEDANCES FOR UNCONDITIONAL AND CONDITIONAL PARAMETRIC-NORMAL VAR USING ROLLING BI-WEEKLY RETURNS

This table contains the statistics on the Asia 50 Price Index for the period January 1993 until January 1998 using 1293 rolling bi-Weekly total returns. The forecasts are based on yearly samples of daily data (260 returns), and a decay factor of 0.94 is used for the $\operatorname{IGARCH}(1,1)$ model for conditional volatility.

\section{Exceedances of Parametric-Normal VaR At 99\% Confidence Level}

Number of Exceedances

\section{THEORETICAL UNCONDITIONAL CONDITIONAL}

\begin{tabular}{lccc} 
Whole Sample & $\mathbf{1 0 . 3 3}$ & $\mathbf{6 2}$ & $\mathbf{7 5}$ \\
$1 / 1994-1 / 1997$ & 7.73 & 13 & 45 \\
$1 / 1997-1 / 1998$ & 2.60 & 49 & 30 \\
\hline
\end{tabular}

\section{Percentages}

\section{THEORETICAL UNCONDITIONAL CONDITIONAL}

Whole Sample

\begin{tabular}{lrrr}
$1 / 1994-1 / 1997$ & $1.00 \%$ & $1.6818 \%$ & $5.8215 \%$ \\
$1 / 1997-1 / 1998$ & $1.00 \%$ & $18.7739 \%$ & $11.4943 \%$ \\
\hline
\end{tabular}




\section{Chapter 5: Times of crisis: A conditional or unconditional approach?}

Unfortunately tail fatness is not captured completely by the use of conditional volatility. This can be seen for example by comparing how forecasts of bi-weekly VaR for the Asian market index from the two approaches compare to their theoretical values over time. We provide rolling bi-weekly forecasts, using an empirical sample of 260 trading days to provide the 10-day VaR, as recommended by the Basle Committee ${ }^{42}$. By undergoing such an out-of-sample test we find that the both the unconditional parametric-normal and the conditional approach using the RiskMetrics ${ }^{\mathrm{TM}}$ methodology would have vastly under predicted the VaR at the $99 \%$ confidence level. The forecasts are based on data from the year prior, and the exact number of exceedances are provided against their theoretical values in Table 5.3 .

Over the whole sample however the unconditional approach would have been slightly more reliable than the conditional approach with actual bi-weekly returns exceeding the biweekly forecast of the VaR $6.00 \%$ of the time rather than $7.26 \%$ of the time. We see that the benefit from allowing volatility to be conditional is only valid when taking the period of financial turmoil alone, with the $18.77 \%$ of exceedances from using unconditional VaR dropping to $11.49 \%$ of the time when using the RiskMetrics ${ }^{\mathrm{TM}}$ conditional approach.

Since the actual bi-weekly returns exceed the VaR forecasts for the $99 \%$ confidence level more often than the theoretical $1 \%$ of the time, it would appear that the assumption of normality results in conditional volatility failing to capture all of the risk. The degree to which normality fails becomes quite dramatic during periods of financial crises, when risk

42 For an analysis of the Internal Model Based Approach to Market Risk Capital Requirement see the Basle reports 
Chapter 5: Times of crisis: A conditional or unconditional approach?

management should become even more conservative. We shall first investigate the nature of this additional downside risk using Extreme Value Theory (EVT), and then shall show how EVT can be used to provide unconditional and conditional VaR-x estimates, which shall be compared to both the unconditional parametric-normal approach and to RiskMetrics $^{\mathrm{TM}}$. The use of EVT enables us to estimate VaR-x and hence capture the additional downside risk faced in times of financial crises.

\subsection{DOWNSIDE RISK AND FINANCIAL CRISES}

Volatility is highly important as a risk measure, especially during periods of financial turmoil. However we have seen that during the turmoil period on Asian markets in 1997, even the conditional normal under-predicts the actual VaR at high quantiles. Bollerslev (1986) also provides evidence that estimating volatility conditionally does not fully capture fat tailedness in asset prices, resulting in underestimation in the VaR at high quantiles. This would imply the existence of additional downside risk. Risk, which becomes more severe during periods of financial turmoil.

Deviations from normality would imply a movement away from the mean-variance framework, and the inclusion of higher moments of the distribution into risk management. Intuitively any additional downside risk should be captured by the extent to which the left tail of the return distribution deviates from conditional normality ${ }^{43}$. An estimate for the tail

43 Of course if a short position is held then the concern becomes that of the right tail of the distribution. 


\section{Chapter 5: Times of crisis: A conditional or unconditional approach?}

index of this left tail, through the use of Extreme Value Theory, will help us not only to capture tail fatness, but also indirectly capture any skewness that the distribution may have.

\section{2. 1 TAIL INDEX ESTIMATION.}

We use Extreme Value Theory to provide us with estimates of tail indices. EVT looks specifically at the distribution of the returns in the tails, and the tail fatness of the distribution is reflected by the tail index. This was first introduced by Hill (1975), and measures the speed with which the distributionís tail approaches zero. The fatter the tail the slower the speed and the lower the tail index given. An important feature about the tail index is that it equals the number of existing moments for the distribution. A tail index estimate equal to 2 therefore reveals that both the first and second moments exist, in that case the mean and the variance; however higher moments will be infinite. By definition the tail index for the normal distribution equals infinity since all moments exist. Since the number of degrees of freedom reflects the number of existing moments, the tail index can thus be used as a parameter for the number of degrees of freedom to parameterise the Student-t distribution. Hence the link to the Student-t distribution, a fatter tailed distribution, which also nests the normal distribution, which we use in VaR-x.

To obtain tail index estimates we use a modified version of the Hill estimator, developed by Huisman et al (1997). Their estimator has been modified to account for the bias in the Hill estimator, with the additional advantage of producing almost unbiased estimates in relatively small samples. Specifying $\mathrm{k}$ as the number of tail observations, and ordering 
Chapter 5: Times of crisis: A conditional or unconditional approach?

their absolute values as an increasing function of size, we obtain the tail estimator proposed by Hill. This is denoted below by $\gamma$ and is the inverse of $\alpha$;

$$
\gamma(k)=\frac{1}{k} \sum_{j=1}^{k} \ln \left(x_{n-j+1}\right)-\ln \left(x_{n-k}\right)
$$

Following the methodology of Huisman et al. (1997), we can use a modified version of the Hill estimator (1997) to correct for the bias in small samples. The bias of the Hill estimator stems from the fact that the bias is a function of the sample size. A bias corrected tail index is therefore obtained by observing the bias of the Hill estimator as the number of tail observations increases up until $\kappa$, whereby $\kappa$ is equal to half of the sample size:

$$
\gamma(k)=\beta_{0}+\beta_{1} k+\varepsilon(k), k=1 \ldots \kappa
$$

The optimal estimate for the tail index, is the intercept $\beta_{0}$. And the $\alpha$ estimate is just the inverse of this estimate. This is the estimate of the tail index that we use to parameterise the Student-t distribution. 
Chapter 5: Times of crisis: A conditional or unconditional approach?

\section{TABLE 5. 4 TAIL INDEX ESTIMATES FOR ASIA 50 INDEX AND SUB- SAMPLES}

This table contains the statistics on the Asia 50 Price Index for the whole period January 1993 until January 1998 using a total of 1302 daily returns, and for the individual years, using an average 259 daily returns. The alpha estimate is calculated using a modified version of the Hill estimator for the tail indexes and is presented for the left tail.

$\begin{array}{ccccccc}\text { ASIA 50 } & \text { WHOLE } & 1993 & 1994 & 1995 & 1996 & 1997 \\ \text { INDEX } & \text { SAMPLE } & & & & & \end{array}$

$\begin{array}{lllllll}\text { Gamma Left Tail } & 0.291 & 0.164 & 0.280 & 0.174 & 0.162 & 0.298 \\ \text { Standard Error } & 0.036 & 0.047 & 0.084 & 0.048 & 0.045 & 0.090\end{array}$

$\begin{array}{lllllll}\text { Alpha Left Tail } & 3.436 & 6.103 & 3.567 & 5.754 & 6.186 & 3.357\end{array}$

Observations

329

67

61

71

71

60

Looking first at the alpha estimates for the whole sample and the individual years in Table

5. 4, we see that the alpha estimates become smaller the larger the deviation from normality. This would imply that the tail index is able to capture some of the additional downside risk. In Figure 5.2 the inverse alpha estimates using the previous years sample of daily data are plotted next to the actual daily returns. We see how that the more the returns fluctuate the higher the inverse alpha estimate and the greater the deviation from 
Chapter 5: Times of crisis: A conditional or unconditional approach?

normality. Indeed the correlation between volatility and alpha is ñ 0.429 , which is highly significant at the $95 \%$ confidence level.

Since we observe fatter tails during periods of instability, we would expect that the use of the alpha estimates in our VaR-x framework to provide more accurate estimates of the VaR during periods of high volatility, especially during financial crises. We now develop the VaR-x methodology to account for conditionality, to see if we are able to capture any of the additional downside risk, crucially important during periods of financial turmoil.

\subsubsection{CONDITIONAL VAR-X}

To capture the existence of any non-normalities in the data into Value-at-Risk we use the VaR-x approach. This allows us to move away from the mean-variance framework and the assumption of normally distributed returns, allowing the return distribution to be fatter tailed if the data exhibits more frequent negative returns than predicted under the normal distribution. The additional parameter, the alpha estimate, for the left tail of the distribution, is then used to parameterise the Student-t distribution. To be able to compare the approach with the RiskMetrics ${ }^{\mathrm{TM}}$ methodology we thus use the same $\operatorname{IGARCH}(1,1)$ model for estimating conditional volatility. However instead of assuming normality we use the standard Student-t distribution, parameterised by the tail index. This enables us to estimate $\mathrm{S}^{*}$, the point on the distribution at which the area 1-c falls to the left. This value then needs to be converted from its zero mean and variance of $\alpha /(\alpha-2)$, so that we use the scale factor $\theta$ equal to: 
Chapter 5: Times of crisis: A conditional or unconditional approach?

$$
\theta=\frac{\sigma}{\sqrt{\frac{\alpha}{\alpha-2}}}
$$

$\theta$ therefore replaces the standard deviate as the risk measure in equation 5 , and $\mathrm{S}^{*}$ is our desired cut off point on the distribution. This then gives us the required return $\mathrm{R}^{*}$ under the VaR-x formulation as:

$$
\mathrm{R} * \tilde{\mathrm{n}} \mathrm{S} * \theta+\mu
$$

We now need only substitute this value for $\mathrm{R}^{*}$ into equation (5.4) to give us the relative VaR-x, equal to $\mathrm{W}_{0} \mathrm{~S} * \theta$. The formulation allows for the conditional or unconditional estimation of the parameters.

In order to see how the VaR-x approach works in practice, we undergo the same out-ofsample test as we did for the parametric-normal approach earlier. 
Chapter 5: Times of crisis: A conditional or unconditional approach?

\section{FIGURE 5. 3 ROLLING VAR-X AND PARAMETRIC-NORMAL VAR}

\section{ESTIMATES}

The graph depicts how the forecasts of the VaR-x estimates, using the Student-t distribution, compare with forecasts from using the Parametric-Normal VaR approach for the Asia 50 Index. We have used rolling observations of daily data, over the period January 1993 until January 1998 using 1302 rolling Bi-Weekly total returns, to provide forecasts of the Value-at-Risk at the 99\% confidence level. The forecasts are based on yearly samples of daily data, and the alpha estimate is calculated for the left tail using a modified version of the Hill estimator.

\section{Bi-weekly Forecasts of Unconditional VaR for the Asia 50 Daily Index 260 rolling observations at the $99 \%$ quantile}

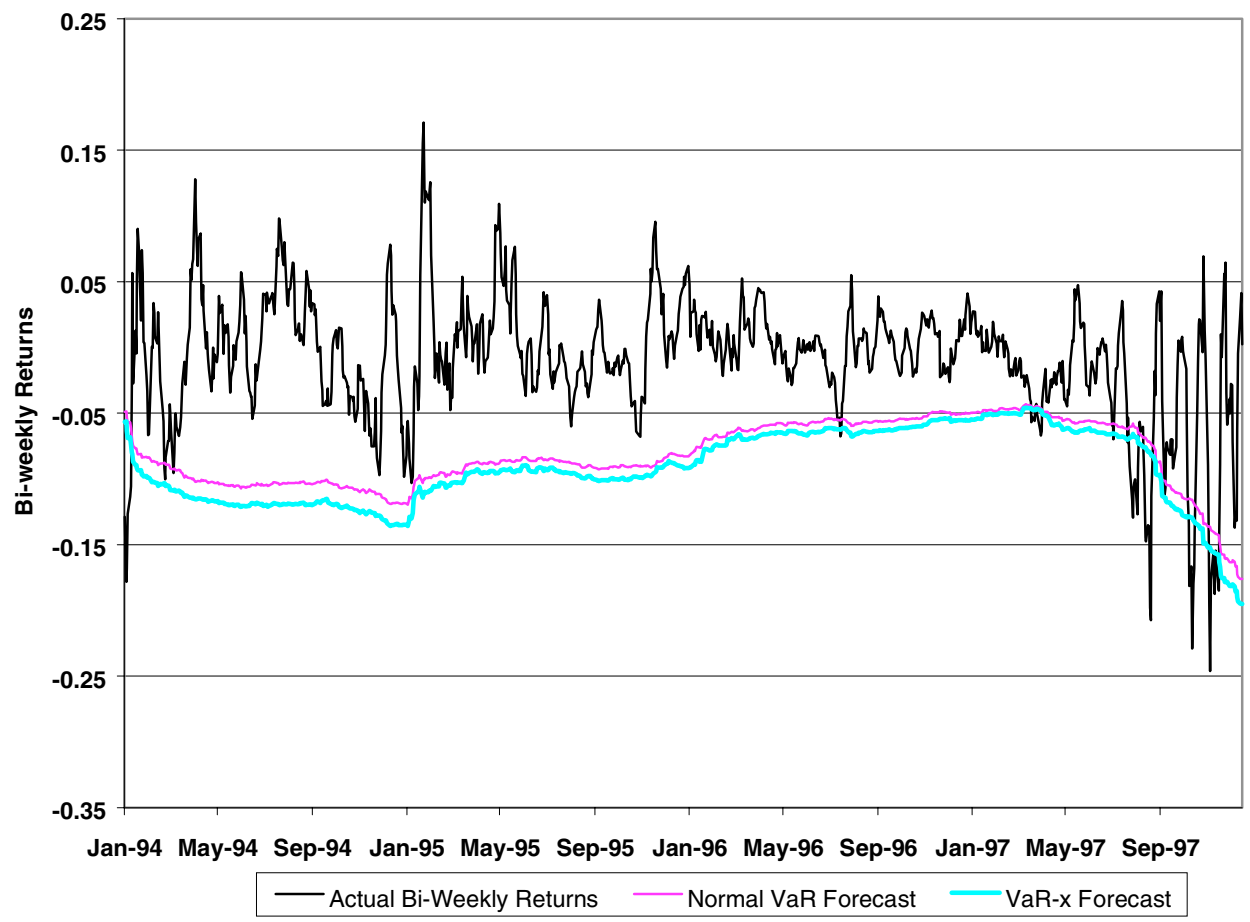


Chapter 5: Times of crisis: A conditional or unconditional approach?

\section{FIGURE 5. 4 ROLLING CONDITIONAL VAR-X AND RISKMETRICTS ${ }^{\text {TM }}$}

\section{ESTIMATES}

The graph depicts how the forecasts of the conditional VaR-x estimates, using the Student-t distribution, compare with forecasts from using the RiskMetrics ${ }^{T M}$ approach of conditional Parametric-Normal VaR for the Asia 50 Index. We have used rolling observations of daily data, over the period January 1993 until January 1998 using 1302 rolling Bi-Weekly total returns, to provide forecasts of the Value-at-Risk at the 99\% confidence level. The forecasts are based on yearly samples of daily data. The conditional volatility is estimated using an IGARCH(1,1) model with a decay factor of 0.94 for the daily data. The alpha estimate is calculated for the left tail using a modified version of the Hill estimator.

\section{Bi-weekly Forecasts of Conditional VaR for the Asia 50 Daily Index 260 rolling observations at the $99 \%$ quantile}

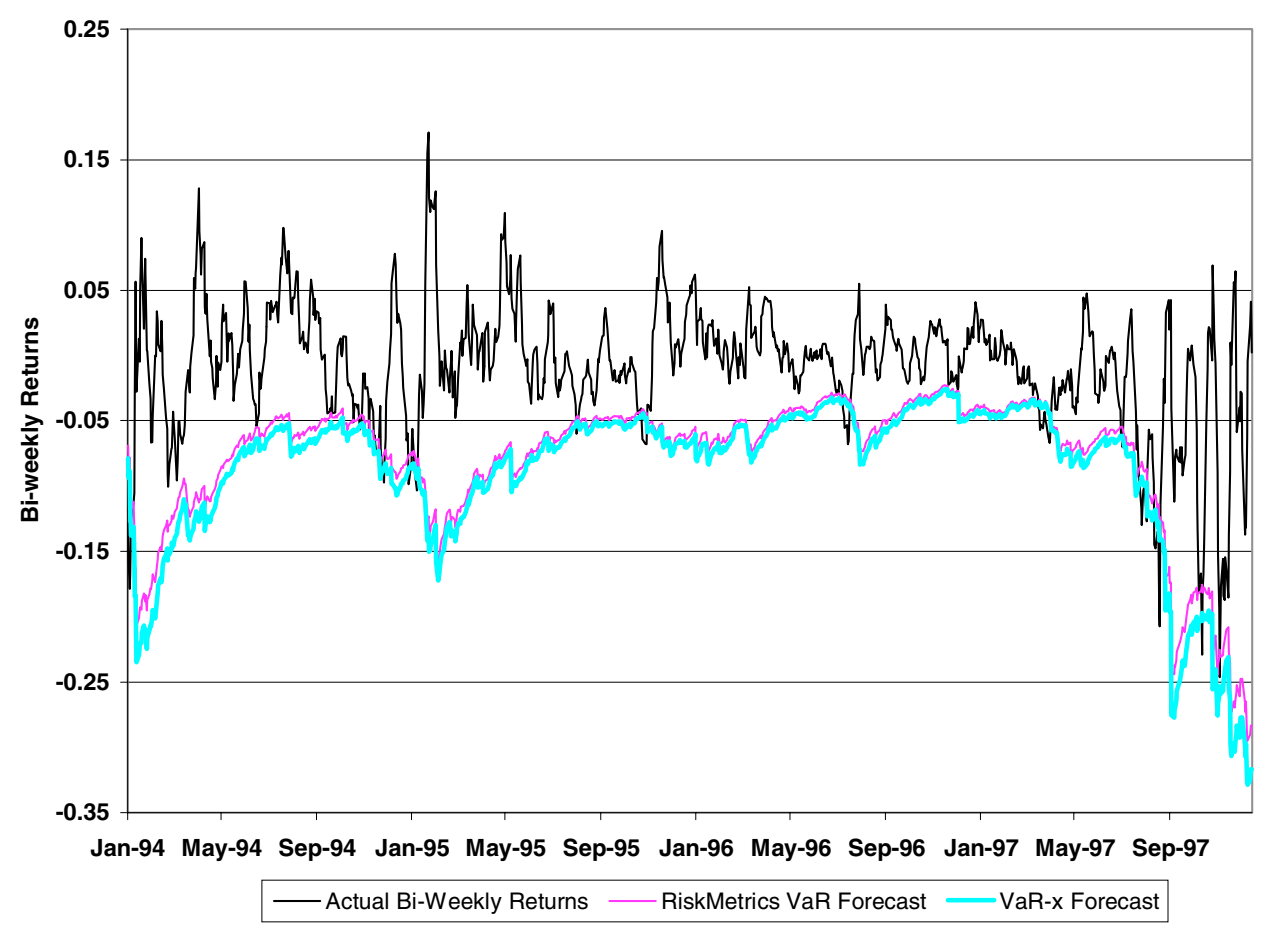


Chapter 5: Times of crisis: A conditional or unconditional approach?

In Figures 5.3 and 5.4 we have plotted the unconditional and conditional forecasts using the VaR-x methodology alongside those generated from the assumption of normally, and conditionally normally distributed returns. We see that the VaR-x approach is able to capture some of the additional downside risk faced in the more volatile periods, beyond that from the use of the standard deviation alone. This is exemplified by the fact that the $99 \%$ boundary lies below that from the parametric-normal approach. During the financial crises the VaR-x approach provides a $99 \%$ boundary even further below that from the assumption of normality, with the difference becoming greater through the use of conditional volatility. In both cases the VaR-x approach provides consistently more accurate forecasts of the VaR than the parametric-normal approach. The number of exceedances and the percentages compared with their theoretical values are presented in Table 5. 5.

Since conditional estimation of $\mathrm{VaR}$ is better able to capture external shocks to volatility during periods of greater turmoil in financial markets, it would seem more appropriate to use a conditional approach to forecasting VaR. The evidence that estimating volatility unconditionally provides more robust forecasts over the entire sample lends itself to the notion that the decay factor in the conditional approach is too low, with the effect of the persistence of a shock dying out too fast. The IGARCH model using a higher decay factor, and hence allowing for external shocks to volatility to persist for longer may therefore be more appropriate. 
Chapter 5: Times of crisis: A conditional or unconditional approach?

\title{
TABLE 5. 5 NUMBER OF EXCEEDANCES FOR UNCONDITIONAL AND
}

\section{CONDITIONAL VAR-X USING ROLLING BI-WEEKLY RETURNS}

This table contains the statistics on the Asia 50 Price Index for the period January 1993 until January 1998 using 1293 rolling bi-Weekly total returns. The forecasts are based on yearly samples of daily data (260 returns), and a decay factor of 0.94 is used for the IGARCH(1,1) model for conditional volatility.

\section{Exceedances of VaR-x At 99\% Confidence Level}

\author{
NuMBER OF EXCEEDANCES
}

\section{Theoretical UnCONDITIONAL CONDITIONAL}

$\begin{array}{lccc}\text { Whole Sample } & \mathbf{1 0 . 3 3} & \mathbf{5 3} & \mathbf{5 5} \\ 1 / 1994-1 / 1997 & 7.73 & 9 & 29 \\ 1 / 1997-1 / 1998 & 2.60 & 44 & 26\end{array}$

\section{Percentages}

THEORETICAL UNCONDITIONAL CONDITIONAL

\begin{tabular}{llcc} 
Whole Sample & $\mathbf{1 . 0 0 \%}$ & $\mathbf{5 . 1 3 0 7 \%}$ & $\mathbf{5 . 3 2 4 3 \%}$ \\
\hline $\mathbf{1 / 1 9 9 4 - 1 / 1 9 9 7}$ & $1.00 \%$ & $1.1643 \%$ & $3.7516 \%$ \\
\hline $1 / 1997-1 / 1998$ & $1.00 \%$ & $16.8582 \%$ & $9.9617 \%$ \\
\hline
\end{tabular}


Chapter 5: Times of crisis: A conditional or unconditional approach?

\section{$\begin{array}{lllllll}\text { TABLE } & 5 . & 6 & \text { IMPROVEMENT OF CONDITIONAL VAR-X OVER }\end{array}$ RISKMETRICS ${ }^{\text {TM }}$}

This table contains the percentage improvement from using conditional VaR-x over RiskMetrics ${ }^{T M}$ for the Asia 50 Price Index for the period January 1993 until January 1998. We use 1293 rolling bi-Weekly total returns and the forecasts using the two approaches are based on yearly samples of daily data (260 returns). For both approaches a decay factor of 0.94 is used for the IGARCH(1,1) model for conditional volatility.

\section{Exceedances of VaR-x At 99\% Confidence Level}

\section{Percentage Improvement from RiskMetrics ${ }^{\mathrm{TM}}$ to VaR-x}

\begin{tabular}{cc} 
Whole Sample & $\mathbf{2 6 . 6 7 \%}$ \\
\hline $1 / 1994-1 / 1997$ & $35.56 \%$ \\
\hline $1 / 1997-1 / 1998$ & $13.33 \%$
\end{tabular}

Of crucial importance however, is that the use of an additional parameter in the VaR-x methodology, to capture the additional downside risk resulting from non-normality, results in more accurate VaR estimates than those from the assumption of conditional normality. When adopting conditional volatility into the VaR-x approach, using the same decay factor for the IGARCH$(1,1)$ model for conditional volatility, we find that conditional VaR-x improves upon the RiskMetrics ${ }^{\mathrm{TM}}$ estimates by over $13 \%$ for the period of financial 


\section{Chapter 5: Times of crisis: A conditional or unconditional approach?}

turmoil $^{44}$. The improvement is even larger when looking at the period as a whole, as shown in Table 5. 6, hence providing much more accurate forecasts of the true Value-at-Risk. It would appear that even though conditional VaR-x is an improvement over the RiskMetrics methodology, further research into additional risk factors is still needed to try to explain the deviations from the use of a conditional student-t distribution to the true distribution.

\section{3. IMPLICATIONS FOR PORTFOLIO SELECTION}

Asian financial markets have been characterised as highly volatile; however they are attractive to international investors since they offer the possibility of achieving high returns with low correlation to developed countriesí returns. The diversification benefits from investing in these markets are thought to be huge. In practice however, we have seen that investors have shied away from these diversification benefits with the potential to diversify having been severely under exploited. Assuming that investors behave rationally, then the lack of diversification can only be explained by assuming that the perceived risk from investing in emerging markets must be much higher than the standard measure of risk suggests. Our evidence that the downside risk involved from non-normality is an important additional risk factor may be of significance when looking at risk premia. If the risk is higher than otherwise assumed under the use of volatility alone, then such a high risk aversion parameter would not be required to explain the huge tendency, shown by investors, towards favouring the home country when investing. The implications for

4 Danielson and de Vries (1997) also use EVT to find a semi-parametric method for estimating VaR, and also find that RiskMetrics under-predicts the true VaR at the $99 \%$ level. Their approach however only performs better far out in the tails, and has the unfortunate drawback of requiring an extremely large sample of data, using 100000 observations. Their analysis is therefore only applicable when high frequency data series is available. 
Chapter 5: Times of crisis: A conditional or unconditional approach?

including non-normality would therefore also have serious implications for the equity premium puzzle, whereby the risk involved in holding equity may be much higher than originally perceived.

The importance of non-normalities is also underlined when observing how any tail fatness, as captured by the tail index, appears to dominate a portfolioís distribution. This results in tail fatness becoming important in a portfolio context. We can see that the tail-index for the Asia 50 Index, when considering the whole sample as given in Table 5. 4, is dominated by the severe deviations from normality occurring during the financial crises in 1997. This highlights the likely importance of additional downside risk in portfolio allocation.

\subsection{CONCLUDING REMARKS}

The empirical evidence on Asian equity markets provides evidence that Asian financial markets have been experiencing more frequent extreme negative returns than suggested by conditional normality. Moreover the deviations tend to be significantly greater during periods of financial turmoil. Such large deviations from normality, whether occurring in response to external economic and political news, or from the presence of herd-behaviour by investors ${ }^{45}$, results in the true risk from exposure on Asian financial markets being underestimated. The implications have already been severely felt, and indeed pose an even more serious threat to the stability of the entire financial system, when considering the contagious nature of financial distress. In order to ensure a sound financial environment it 


\section{Chapter 5: Times of crisis: A conditional or unconditional approach?}

is crucial that risk management techniques accurately reflect the risk from exposure to financial markets. It therefore is of vital importance that the effects from additional downside risk are included into risk management.

Our conditional VaR-x estimates more accurately capture the additional risk, reflected by increased tail fatness, particularly crucial in times of financial crises. This has been shown with respect to the RiskMetrics ${ }^{\mathrm{TM}}$ methodology, which on testing out of sample resulted in a significant improvement to accurately forecasting Value-at-Risk. Accurate measurement of this exposure is crucial for financial risk management, both from an institutionís, and a regulatorís, point of view. Institutions should be required to hold more capital, and if insurance schemes are set $u^{46}$, then the premium paid to regulators would need to be greater. Such measures should result in greater financial stability, with fewer banking and security house failures within Asian markets. Indeed it has been shown that those firms which have held a more conservative position regarding their capital standards have been able to dodge major losses from the turmoil across $\mathrm{Asia}^{47}$.

The importance of downside risk appears to play a crucial role in the improvement of risk management techniques and the avoidance of further financial crises. Our VaR-x approach not only highlights the huge risks and potential danger from investing in Asian markets, but also directs attention towards the importance of deviations from normality in asset

\footnotetext{
4 See Kaminsky and Schmukler (1998), for an interpretation on the causes leading to such extreme market returns in Asia.

46 A proposed International Credit Insurance Corporation for example, as proposed by George Soros (EIU 1998a), would require the purchase of an option in the form of an option form the agency, with the greater the firm's risk exposure the greater the fee to be paid.

47 See EIU (1998b) for a detailed survey of the response of major international firms to financial turmoil in Asia.
} 
Chapter 5: Times of crisis: A conditional or unconditional approach?

pricing theory. The additional risk faced by the investor may indeed be able to give a better understanding of the size of the risk premium. This points towards an explanation of both the high equity premium and home-country bias, without having to resort to excessive estimates of peoples risk aversion. The implications for portfolio management are therefore of particular interest for future research. 
Chapter 6: Covariance and correlation

\section{CHAPTER 6 COVARIANCE AND CORRELATION ${ }^{48}$}

Recent discussion on the correlation of international asset returns has focussed on the issue of whether extreme movements in international financial markets are more highly correlated than usual returns. This implies a reduction in the benefits arising from portfolio diversification since extreme returns are more likely to occur with greater simultaneity. Using the Value-at-Risk methodology we are able to measure the quantile correlation structure implicit in international asset returns in a simple, non-spurious, manner. We find that for extreme movements in international equity markets the assumption of bivariate normality reduces the benefits of international diversification even after discarding spurious correlation changes.

48 This chapter is based on R. A. Campbell, K.G. Koedijk and P. Kofman, 'Increased Correlation in Bear Markets: A Downside Risk Perspective', Forthcoming in the Financial Analysts Journal (2001). 
Correlation estimates form the core of the analysis of the risk-return trade off associated with investment portfolios. Low correlation is desirable from an investment perspective with diversification benefits materialising when a fall in one market is offset by a rise in another market. However, if there is a tendency for all markets to fall simultaneously then the benefits from diversification will be overstated. Recent research has investigated the phenomenon of whether correlation has changed over time. This literature has generally adopted either multivariate GARCH or regime switching models, which are able to capture the time-varying nature of volatility and correlation measures. Time-varying correlation estimates have been found to have increased over more recent sample periods (Longin and Solnik (1995) and Karolyi and Stulz (1996)). While the observation of time-varying correlation does not necessarily imply size-dependent correlation, correlation was found to increase particularly in periods when bear market conditions prevailed.

Correlation estimates that are conditional on the size of market movements are of considerable relevance to investment analysis, since it is in times of more extreme market conditions when the benefits from diversification (and the effect of low correlations) are most urgently needed. A number of studies have dealt with the estimation of sizedependent correlation. (See Ramchand and Susmel (1998), Longin and Solnik (2001), Boyer, Gibson and Loretan (2000), and Loretan and English (2000)). In all of these studies, a correlation structure is estimated conditional on market returns falling below (or above) a pre-specified return level. The conditioning either occurs on a single component or occurs on both components of the joint return distribution. 


\section{Chapter 6: Covariance and correlation}

Such approaches to estimate size-dependent correlation invariably suffer from a theoretical estimation bias invalidating the estimates from a practical perspective. Properly adjusting for this bias is therefore required before applying the estimates to investment analysis. As it turns out, the bias not only depends on the choice of extreme returns (i.e., how the sizeconditioning occurs), but also on the assumed underlying joint return distribution. Boyer, Gibson and Loretan (1999) and Loretan and English (2000), for example, condition the correlation estimates on one component of the bivariate normal return distribution. The truncated conditional correlation is then estimated for bivariate return observations where one of the returns exceeds a certain threshold level. Unfortunately, one cannot straightforwardly compare these conditional truncated correlation estimates with the unconditional correlation estimate to draw conclusions with regard to constant correlations in the tails of the joint return distributions. By truncating a joint return distribution with constant correlation, the conditional truncated correlation will be biased downwards for increasing threshold levels (i.e., more extreme market conditions). The qualitative implication of this is that one should reject constant correlation more frequently. Butler and Joaquin (2000) also use this approach and correct their estimates for estimates of the Sharpe ratio. However, when we drop the normality assumption in favour of a fatter tailed alternative bivariate distribution, the picture is quite different. Now, the truncation causes an upward bias for more extreme market movements. Hence, we should not reject constant correlation as easily. This approach is discussed and illustrated for the Student-t distribution in detail in Campbell, Forbes, Koedijk and Kofman (2001).

An alternative approach, which Longin and Solnik (2001) follow, is to estimate conditional correlation where conditioning occurs on both return series falling below (or above) pre- 
specified return levels. By considering only joint threshold exceedances, we obtain a ëquadrantí of relevant return observations. When moving into the bivariate tail of the distribution we include only those joint return observations that fall into smaller and smaller quadrants of a scatter plot of the joint returns. The conditional correlation structure from such an approach converges to zero as we move further into either tail of the distribution. Hence, a somewhat similar theoretical bias occurs simply because of the way the conditioning extreme observations are defined.

Portfolio and risk managers alike require a meaningful yet practical interpretation of the conditional correlation structure of the joint return distributions of global financial assets. In order to apply conditional correlation estimates to investment analysis, the conditional correlation structure needs to be adjusted to account for the theoretical estimation bias and therefore requires knowledge of how the conditioning occurred. Ang and Chen (2000), for example, correct for the bias induced by both single and joint component conditioning techniques to analyse asymmetric correlations in the US equity markets. A thorough understanding of the conditioning technique used to obtain size-dependent estimates is therefore crucial before considering application of any size conditional correlation measure. However, for a size-dependent correlation measure to be of practical use to portfolio analysis or in risk management, it should relate to the correlation measure typically used by practitioners. Preferably such a conditional correlation measure should be independent of how the conditioning occurs. 


\section{Chapter 6: Covariance and correlation}

Therefore, instead of adjusting conditional correlation estimates for the theoretical bias, we propose an alternative conditioning approach to estimate the conditional correlation structure. Rather than focussing on truncated or quadrant conditioning, we condition the correlation measure in a manner consistent with portfolio Value-at-Risk. The advantage is that our correlation estimates for portfolio returns are conditioned on portfolio returns falling below a pre-specified ëworst-caseí portfolio quantile, instead of conditioning on one or both of the assetsí returns comprising this portfolio. This has a number of advantages. Firstly the approach is in keeping with how correlation is applied in both portfolio management and in risk management. Our correlation measures can directly be applied to both Markowitz style portfolio optimisation and to Value-at-Risk analysis. Secondly, the approach easily generalizes from the bivariate to the multivariate scenario. By using portfolio returns we effectively collapse the multi-dimensional case into a one-dimensional case, i.e., a univariate portfolio returns distribution. A third advantage is the characterisation of the conditional correlation structure thus derived. For a broad class of elliptic distributions with constant correlation, we observe that the theoretical conditional correlation is equivalent to the theoretical unconditional correlation. Any deviations from this correlation can directly be attributed to size-dependent correlations, without having to worry about measurement bias. Hence, in a sense the measure is ëconditioning-free.í

Using data on international equity markets we observe how the correlation structure between international equity markets changes ëwhen we move throughí the joint return distribution. We find significant evidence of increased correlation in the tails of the joint distributions indicating contagion between financial markets for more extreme market movements. We also observe that this effect is greater for negative movements in equity 
markets, with the influence of greater downside risk becoming more apparent during bear markets. In a simple manner we are therefore able to gauge the effects of a changing correlation structure on the gains to international diversification and assess the implications for modern portfolio theory and risk management.

The outline of the chapter is as follows. In the following section we introduce our sizedependent correlation measure, known as the quantile correlation structure. Section 6.3 contains the empirical application using daily data on various international stock market returns. The implications for portfolio optimisation are discussed in section 6.4 , and conclusions are drawn in the final section.

\subsection{ALTERNATIVE CONDITIONAL CORRELATION STRUCTURE}

In order to determine how the correlation structure changes over the return distribution of two financial time series a decision has to be made on which returns to condition. There are various ways in which a correlation measure conditional on the size of the joint asset returns can be defined. We propose the use of a conditional correlation measure derived from the literature on Value-at-Risk ${ }^{49}(V a R)$. This size-conditional correlation measure follows directly from $\operatorname{VaR}$ measurement, and provides a simple methodology for estimating the correlation structure implicit in joint portfolio returns, without having to resort to Extreme Value Theory (EVT) estimation or fully parametric modelling of the

49 See Jorion (1996), Jorion (1997) and Linsmeier and Pearson (2000) for an introduction and discussion of Value-at-Risk in practice. 


\section{Chapter 6: Covariance and correlation}

joint return distribution. The use of this quantile correlation measure is very much in line with current portfolio management with the correlation measure conditioning on the downside support of the joint distribution of returns.

By using the $\operatorname{VaR}$ methodology we are able to estimate the probability of a portfolioís return falling below a threshold $(\mathrm{VaR})$ return with a prespecified confidence level. Assuming constant correlation over the joint return distribution, the probability of the portfolio return falling below this $V a R$ level is a weighted average of the probabilities of the individual assetsí returns in the portfolio falling below this $V a R$ level. As we increase confidence levels, we move further out into the tails of the joint portfolio distribution, and the $V a R$ increases. However, if at the same time the correlation between the assetsí returns increases, then the $\operatorname{VaR}$ on the portfolio will exceed the weighted average of the individual VaRs. We invert this relationship and by observing the difference between the portfolio $\operatorname{VaR}$ and the $\operatorname{VaR}$ levels for the individual assets in the portfolio, we can determine exactly how the correlation structure changes as we move further out into the tails of the joint return distribution.

$\operatorname{VaR}$ quantile estimation can be summarised in terms of a quantile return $q_{c}$ that will not be exceeded with (1-c)\% probability. Assuming jointly normally distributed returns with mean zero and standard deviation sigma the quantile return is simply a function of the standard deviation of the univariate normal distribution:

$$
q_{c}=\zeta_{c} \sigma
$$


$\zeta_{c}$ is the (1-c)\% quantile of the standardized normal distribution. Writing equation (6.1) as a portfolio quantile, squaring it, and substituting the portfolio variance by its components:

$$
q_{\text {port }, c}^{2}=\zeta_{c}^{2}\left[w_{x}^{2} \sigma_{x}^{2}+w_{y}^{2} \sigma_{y}^{2}+2 w_{x} w_{y} \sigma_{x y}\right]
$$

Replacing the individual standard deviations by their VaR quantile companion estimates gives a conditional correlation measure:

$$
\rho_{Q}=\frac{q_{p o r t, Q}^{2}-w_{x}^{2} q_{x, Q}^{2}-w_{y, Q}^{2} q_{y, Q}^{2}}{2 w_{x} w_{y} q_{x, Q} q_{y, Q}}
$$

We label the measure in (6.3) as the quantile correlation measure. For a normal distribution we can simplify (6.3) such that the quantile correlation is constant for increasing quantiles

$$
\rho_{Q}=\rho
$$

Of course in the case of normality since correlation is bounded by $\tilde{n} 1<\rho<1$, the quantile correlation is also bounded by $\tilde{\mathrm{n}} 1<\rho_{\mathrm{Q}}<1$. 


\subsection{INTERNATIONAL EQUITY MARKETS}

In order to observe how the correlation structure changes over the joint distribution of international equity returns we compare the empirical correlation structure to the (constant) theoretical quantile correlation structure assuming bivariate normal return distributions. This enables us to comprehensively answer the question as to whether large movements (of either sign) in equity markets are more highly correlated than smaller movements. We use daily data from Datastream for the USA, UK, France, Germany and Japan, the same countries as used in Longin and Solnik (2001) but, at a higher sampling frequency. ${ }^{50}$ This data set extends from May 1990 to December 1999, i.e., 2500 observations. We observe that the average return on the S\&P500, FTSE100, CAC40 DAX100 was around 15\% over the sample period, close to twice the return on the 10-year US Datastream Government Bond Index. At the same time, the equity index returns have been two to three times as volatile as the US Government Bond returns.

50 Longin and Solnik (201) use monthly data from 1959 to 1996 


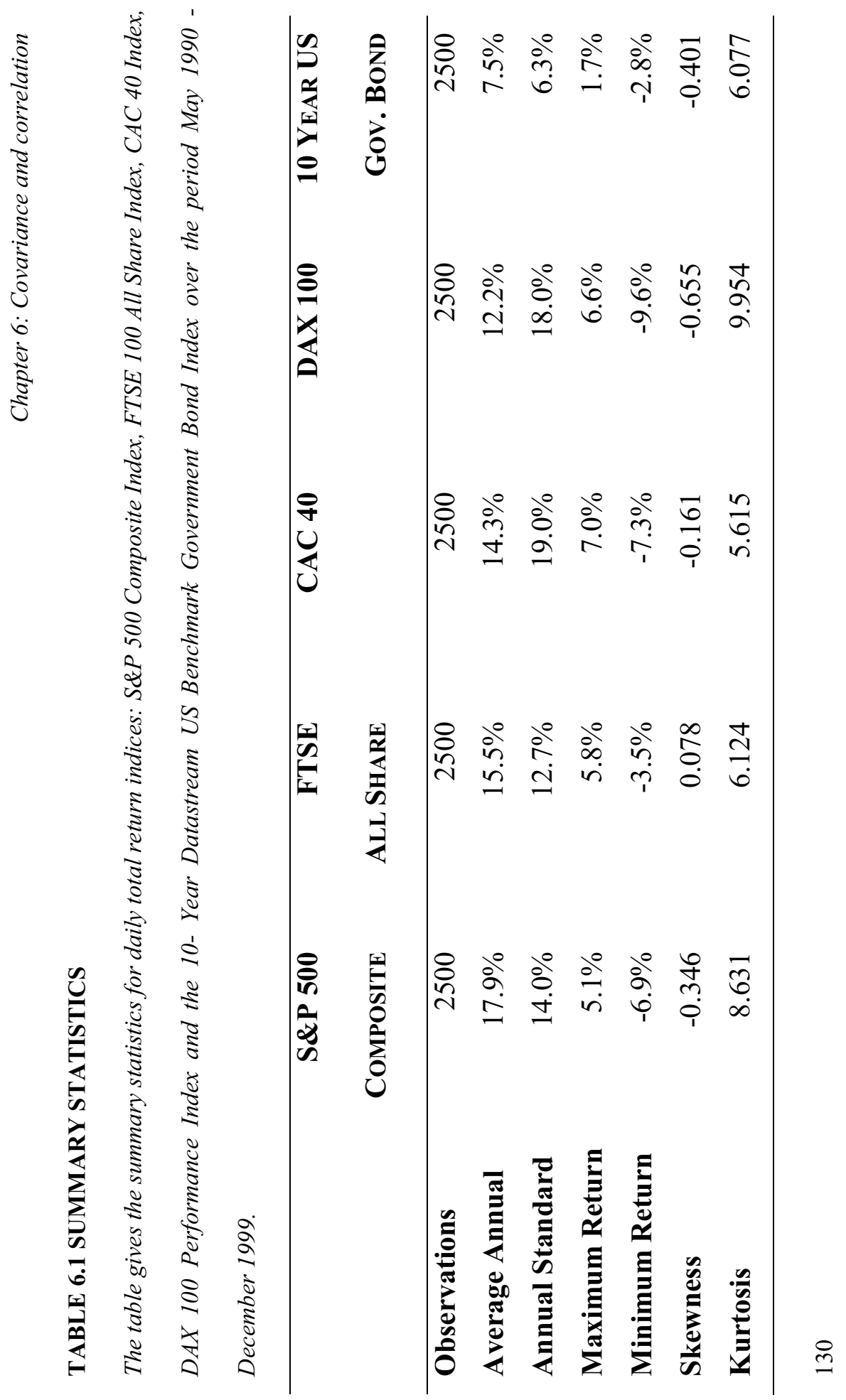




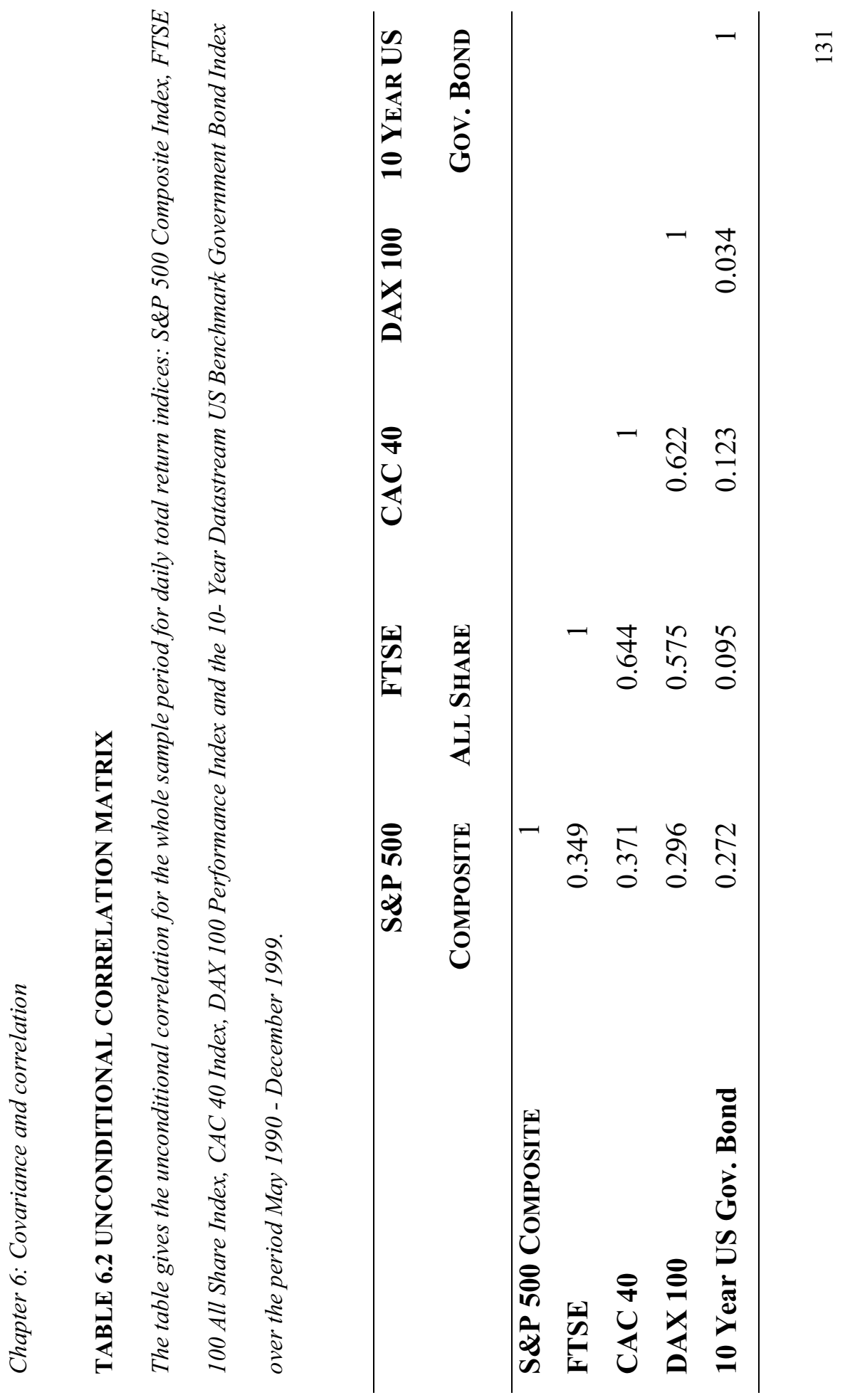


Summary statistics for the data are given in Table 6.1. All of the return series exhibit highly significant excess kurtosis, and all except the FTSE exhibit significant negative skewness. This implies that all the return distributions have greater probability mass in the tails of the distributions than the normal distribution would predict. There is therefore a greater probability of lager movements in the stock and bond markets than the assumption of normally distributed returns would predict. Deviations from normality may have implications for the correlation structure of the bivariate distribution, which we estimate below.

In Table 6.2 we have estimated the unconditional correlation estimates for the various international return index combinations. The unconditional correlation between the S\&P500 and the European stock markets averages 0.338 , whereas the unconditional correlation is much higher between the European markets, averaging 0.620. This greater co-movement in European stock markets results in almost $40 \%\left(0.620^{2}\right)$ of stock price movements being common to European markets, whereas only around $10 \% \quad\left(0.338^{2}\right)$ of stock price movements are common to both the US and European markets. The unconditional correlation is, as expected, considerably lower between stock market and bond market returns; the domestic correlation between the S\&P500 and the 10year US Government bond index is only 0.272 . This correlation falls to an average of a mere 0.084 for the European Markets and US Government Bonds. 


\section{Chapter 6: Covariance and correlation}

Based on the estimates of the unconditional correlations, we can parameterise the bivariate normal distribution. We are now in a position to estimate the conditional quantile correlation structure using the historical data, and compare it to the theoretical quantile correlation structure. In Figure 6.1 we have therefore estimated the empirical quantile correlation structure for the S\&P500 and the FTSE and have plotted it against the theoretical correlation structure assuming bivariate normality for the left tail of the distribution.

We have also plotted $95 \%$ confidence interval for the estimates, and indeed we see that the data only violate the assumption of normality and constant correlation in the tails of the distribution (for quantiles greater than 95\%). Up until the 95\% level, we cannot reject the null of constant correlation. Thereafter (i.e., further out into the tails of the distribution) correlation appears to increase significantly above the unconditional correlation. If the assumption of bivariate normality is reasonable, then allowances would have to be made for greater correlation of large movements in bull markets. We also simulated quantile correlations with bivariate Student-t distributions. The same qualitative results hold for the quantile correlation estimator. The theoretical quantile correlation is still constant and equal to the unconditional correlation. The only difference is that the standard error bands are considerably wider, hence we would not reject constant correlation as frequently. 


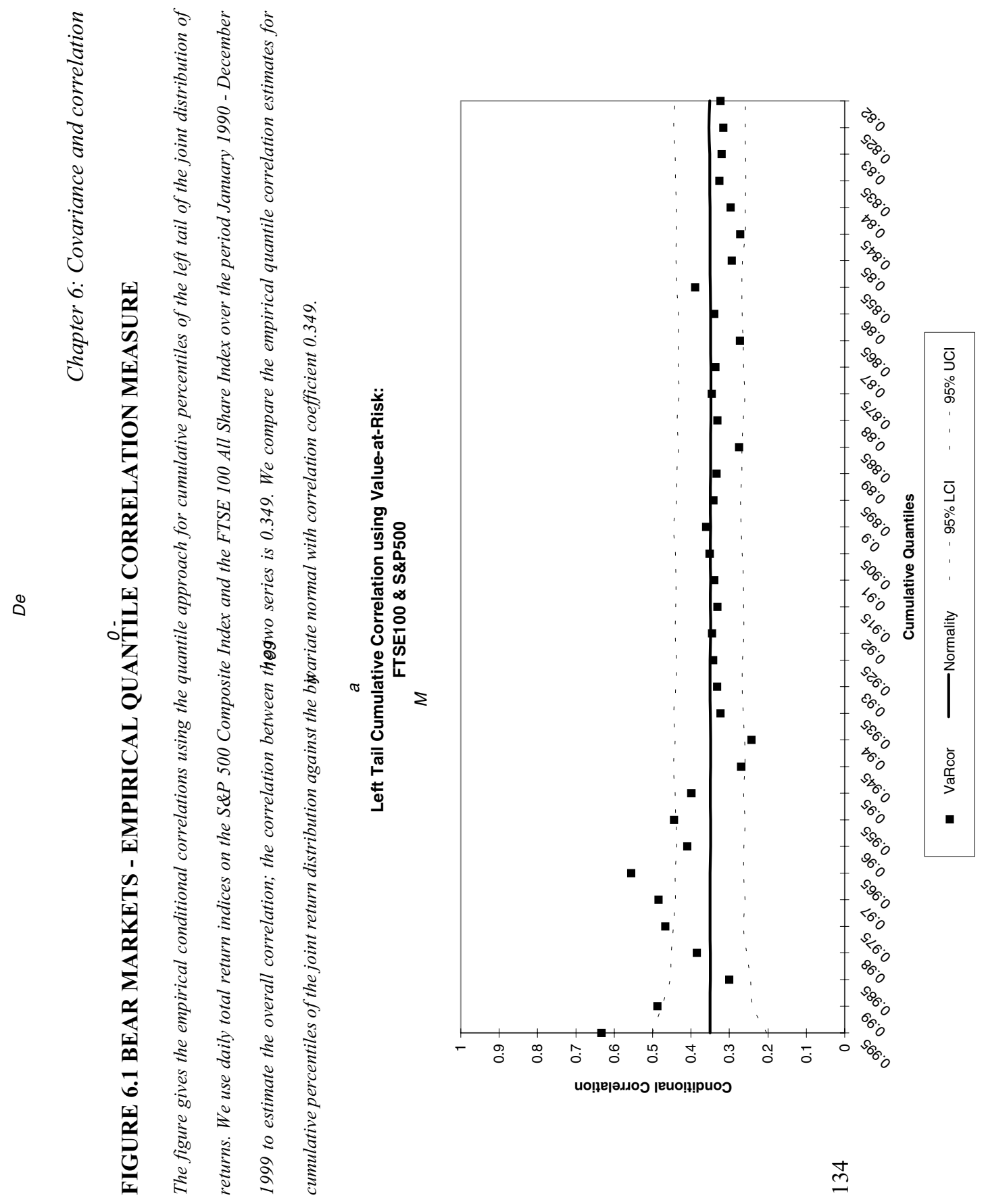




\subsection{IMPLICATIONS FOR PORTFOLIO DIVERSIFICATION}

Having observed an increase in conditional quantile correlation during bull markets, it now seems worthwhile to establish its relevance to the portfolio allocation decision. In modern portfolio theory mean-variance investors maximise expected return for a given level of risk as determined by the variance of the unconditional return distribution. No allowance is made for investors who weigh losses more heavily than gains, or large losses more heavily than smaller losses. Risk is a purely symmetric measure and correlation is assumed to be constant, i.e. size-independent. So even if investors did attribute greater risk to large losses, the assumption of joint normality will still result in the same optimal mean-variance efficient portfolio. However, empirical deviations from normality result in increased probability mass in the tails of the return distribution, and with it an increased correlation between large negative movements in equity markets. This has serious implications for portfolio management.

The benefits to international diversification depend crucially on assets being less than perfectly correlated. These benefits would be severely eroded by increasing correlation between asset returns in the tails of their joint distributions. In fact, when most needed the protection offered by diversification would rapidly erode. For investors worried about greater downside risk from increased conditional correlation, this requires a reformulation of the mean-variance portfolio allocation model. Instead of maximising expected returns given the unconditional variance-covariance matrix, we then maximise expected returns given a tail-adjusted variance-covariance matrix. Once we know the appropriate level of 
downside risk of concern to the investor, we can substitute the unconditional correlations by their conditional quantile correlation equivalents given that level of downside risk. In table 6.3 we show the average percentage increase in correlation (conditional quantile minus unconditional) as required for a range of levels of downside risk.

The results illustrate that for investors or risk managers concerned with downside risk at the $90-95 \%$ level little adjustment in correlation input is required for estimating the riskreturn trade off in global equity markets. The conditional quantile correlation estimates provide evidence of slightly lower correlations between global markets when conditioning on the $90 \%$ to $95 \%$ downside quantile of the joint return distribution, however the decrease is insignificant for all series. Only for the combination of S\&P500 and the CAC40 is there any evidence of increased correlation in this range. In particular the quantile correlation between the US equity and US bond market shows evidence of greater diversification benefits than based on the unconditional correlation estimate. Once we focus on more extreme downside risk, however, there is significant evidence of large increases in the conditional quantile correlation between global equity returns and between bond market returns and global equity returns. The S\&P500 and the FTSE show the greatest increase in conditional quantile correlation, a $31 \%$ increase. For investors and risk managers who require a greater degree of confidence in their portfolio or risk management recommendations, the benefits arising from international diversification are significantly curtailed. 


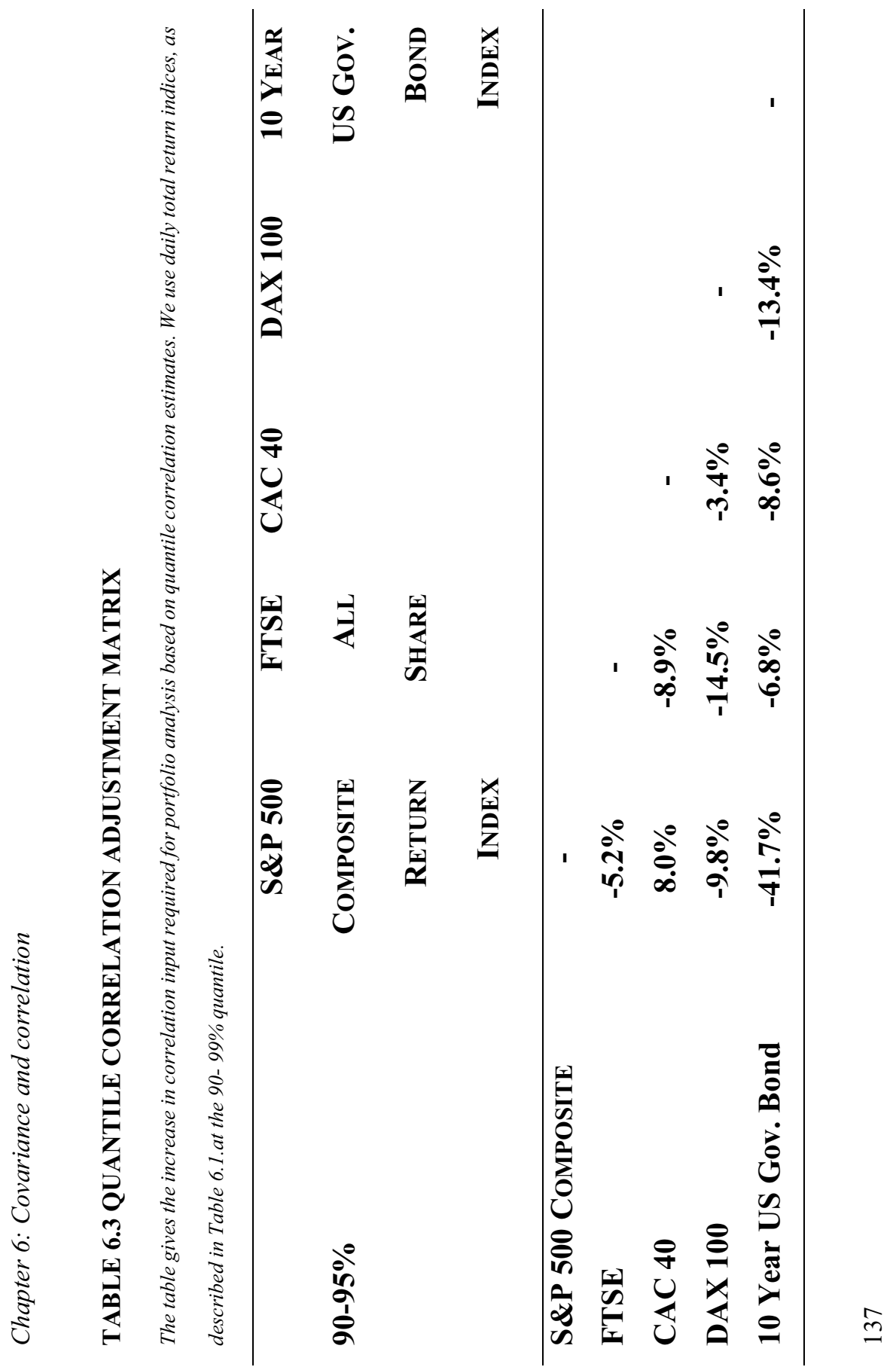




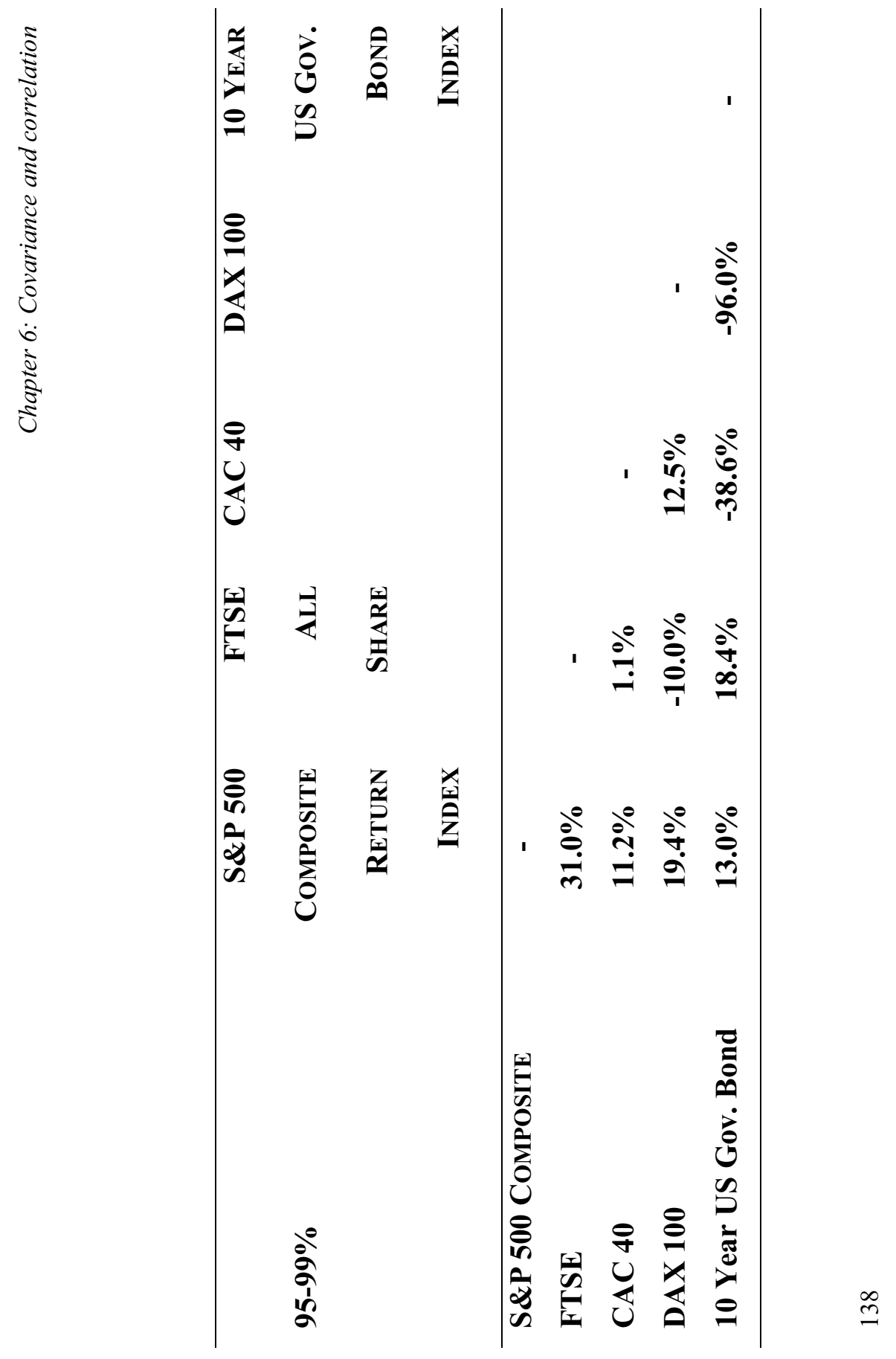




\section{Chapter 6: Covariance and correlation}

In Figure 6.2 we have plotted two efficient frontiers to gauge the trade-off between risk and return as we move from a $100 \%$ investment in the FTSE to a $100 \%$ investment in the S\&P500. The trade-off is significantly greater when using the higher conditional correlation of 0.457 for the $95-99 \%$ quantiles rather than the unconditional correlation of 0.349. To achieve a given amount of return the risk is therefore greater. The implication being greater downside risk during bear markets. It would therefore be better to use conditional mean-variance optimisation under such circumstances. For example assuming a risk-free rate of $7 \%$ the optimum allocation on the efficient frontier using the unconditional correlation measure is a 55\% holding in the S\&P500 and a 45\% holding in the FTSE. To maintain the same level of risk as when using the unconditional correlation measure then a $10.5 \%$ holding in the risk-free rate is required, with a further $50 \%$ in the S\&P500 and 39.5\% in the FTSE. It is during times of extreme bear markets that the effect from diversification is really required and it is therefore crucial that under such market conditions the appropriate correlation estimates are used, and the additional downside risk is incorporated. Such an approach to account for this greater downside risk from increased correlation during bear markets provides the basis for optimisation of a downside risk $\operatorname{model}^{51}$.

51 See Campbell, Huisman and Koedijk (2001) for portfolio optimisation using a downside risk model. 
Chapter 6: Covariance and correlation

\section{FIGURE 6.2 EFFICIENT FRONTIER}

The figure gives the efficient frontier comprising the risk-return trade off on an annual basis for the total return indices on the S\&P 500 Composite Index and the FTSE 100 All Share Index over the period January 1990 December 1999 (See Table 6.1 for a summary of the data).

Efficient Frontier: S\&P500 \& FTSE

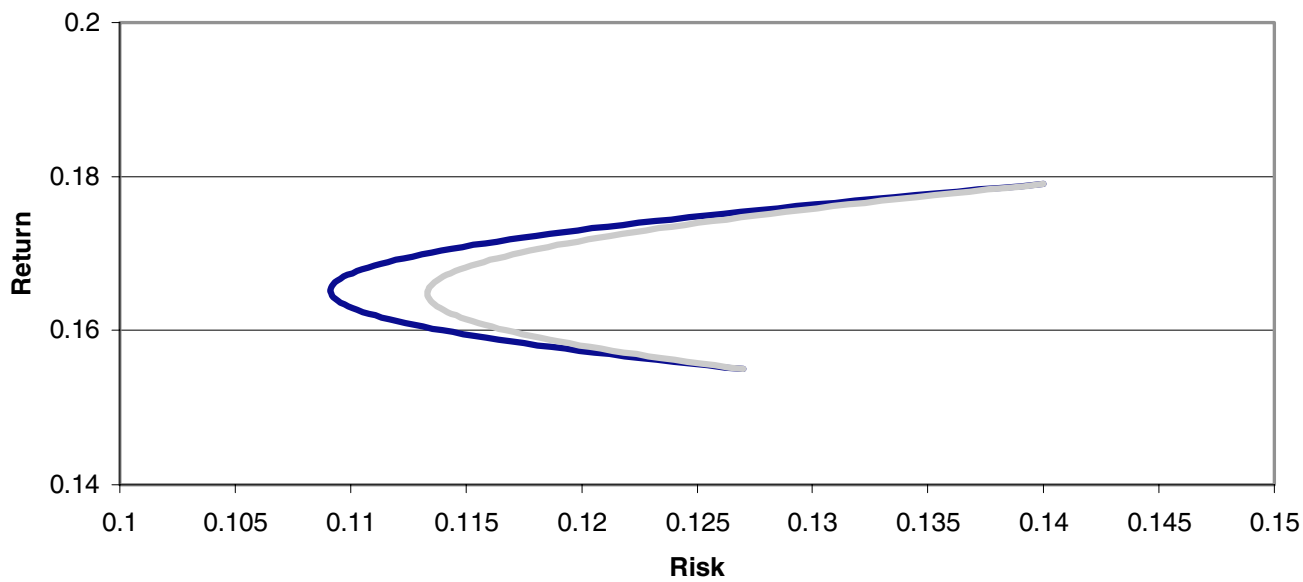

- unconditional correlation $0.349-$ conditional correlation 0.457 


\subsection{CONCLUDING REMARKS}

It is now well established that volatility is time-varying and should be modelled accordingly. In a multivariate context this implies that correlation should also be modelled as a time-dependent variable. Market lore and intuition tell us that the observed timevariation in correlation might also be a proxy for size-dependency in correlation. Large negative returns, in particular, on international equity markets tend to coincide much more frequently than would reasonably be expected from the unconditional return correlation. Early attempts to capture the size-dependency in correlation have so far been hampered by biased estimates and size-conditioning that defies practical use in portfolio allocation. We propose using a correlation estimator that does not suffer from these shortcomings.

Our quantile correlation estimator conditions on the quantiles of the multivariate distribution of portfolio returns. Since these quantiles are well established in the portfolio $\mathrm{VaR}$ literature, they allow the conditional correlation estimator to be analysed in a portfolio context. This makes the quantile correlation measure directly applicable to portfolio allocation and therefore appealing from a practitionerís point of view. A further advantage is its unbiasedness, which allows direct comparison of the conditional quantile correlation with the unconditional correlation. When applied to data on international stock market index returns we find evidence of increasing correlation in the tails, indicating that an amended variance-covariance matrix ought to be used for mean-variance portfolio analysis and risk management techniques when investors are concerned about downside risk. 
In times of financial crises one easily gets the impression of contagion and spillovers occurring across and between financial markets - in excess of normal market conditions. This suggests that financial returns are more highly correlated for large negative returns than for modest returns. Using an alternative estimator for the conditional correlation structure of size-dependent correlation we find that this intuition is indeed corroborated by empirical evidence. The implications for portfolio allocation and risk management are therefore serious since the benefits of diversification are partly eroded when they are needed most. 
Chapter 7: The downside of international equity markets

\section{CHAPTER 7 THE DOWNSIDE OF INTERNATIONAL EQUITY MARKETS ${ }^{52}$}

In this chapter we take an alternative view to asset pricing by focussing on the risk-return trade off with particular attention to downside risk. By moving away from the expected utility framework, and the necessary restrictive assumptions on which consumption based asset pricing is based, we are able to provide some more insight into two finance puzzles which currently dominate the finance literature: the equity premium puzzle and the home bias phenomenon. Using a downside risk portfolio allocation model we use data on international equity markets and provide empirical results as to the extent of the riskreturn trade-off facing investors in international financial markets. The results provide evidence that investors are concerned with the potential downside risk in international equity markets, and by using a confidence level associated with the downside risk to assess investorsi risk aversion levels are able to incorporate some of the findings of behavioural finance into portfolio management theory.

52 This chapter is based on R. A. Campbell, 'The Downside of International Equity Markets, Working Paper (2000). 
Finance theory centers on the prevalence of a risk-return trade off, so that additional returns on financial assets are only gained as the reward for bearing additional risk. It is commonly understood that shares are riskier than safer assets such as bonds; however a number of puzzles have arisen as to the extent of the risk-return trade off in national as well as international financial markets. In finance theory the additional risk borne by investors does not justify the size of the premium for holding equity. In order to explain the equity risk premium within an expected utility framework investors must have extremely high levels of risk aversion to justify the high additional returns made for bearing only a small amount of additional risk. This phenomenon has been commonly dubbed as the equity risk puzzle and was first suggested by Mehra and Prescott (1985).

A similar puzzle evolves as to the extent of the risk-return trade-off faced on international financial markets. Investors have notoriously held a larger proportion of their portfolio in domestic assets, dubbed the home-bias phenomenon, even though modern portfolio theory suggests that greater diversification benefits can be obtained through a larger holding in foreign markets. It would appear that the risk international equities for example possess is greater than captured in current finance theory; so that the modern tools in finance for estimating risk currently under-estimate the true risk to investors of holding international equities.

The literature has however continued to focus on expected utility theory and the consumption based approach to asset pricing as well as the standard deviation as the appropriate measure for risk. Little attention has been given as to the correct definition and 


\section{Chapter 7: The downside of international equity markets}

size of the risk-return trade-off beyond this framework. Indeed the measure for risk is highly debatable, and the puzzle may largely be due to the use of the incorrect measure for risk in financial markets.

In this chapter we assess how the use of a downside measure for risk, such as used in risk management for evaluating market risk, helps us to gain some greater insight into some of the puzzles in the current financial literature. The benefit of using an alternative approach to assessing risk-return trade-offs, enables us to move away from the consumption based approach to asset pricing, and some of the constricting assumptions required for expected utility theory, and provide an alternative view to the risk-return trade-off observed in international financial markets. We hope to provide some insight into these two puzzles currently dominating mainstream finance - the equity premium puzzle and the home bias phenomenon. If such a downside risk measure is able to capture additional downside risk, over that captured by the use of standard deviation alone, then both these phenomena may be explained by the use of downside risk and may merely be a facet of the definition for risk.

The outline of the chapter is as follows. We focus in the following section on asset pricing, and the risk-return trade-off as captured by two alternative approaches to asset pricing: consumption based approach and the downside risk approach, as well as some of the empirical findings in behavioural finance. In section 7.2 using empirical data on international equity markets we model how the downside risk approach assesses the riskreturn trade off for various levels of investorsí risk aversion. We hope to provide some insight into how the downside risk approach is able to provide us with an alternative risk- 
return trade-off for assessing the extent of the equity premium as well as about investorís desires to invest internationally. Conclusions are drawn in the final section, section 7.3.

\subsection{PRICING FINANCIAL ASSETS}

In order to assess risk-return trade-offs on financial markets we first need to provide a general framework within which we can work to compare the various approaches to asset pricing. In this section we therefore follow the methodology of Cochrane (2000). In efficient markets prices on international financial markets should simply reflect the discounted value of their expected future payoffs. Denoting $\mathrm{P}$ as the price of asset $\mathrm{i}$ at time $\mathrm{t}, \mathrm{X}$ as the payoff and $\mathrm{E}$ as the expectations operator, then $\mathrm{M}$ is defined as the so-called stochastic discount factor (SDF). This gives rise to the following accounting identity, which is the heart of asset pricing ${ }^{53}$.

$$
P_{i t}=E_{t}\left[M_{t+1} X_{i, t+}\right]
$$

This can also be written in terms of returns, $\mathrm{R}$ :

$$
1=E_{t}\left\lfloor M_{t+1} R_{i, r+1}\right\rfloor
$$

\footnotetext{
See Cochrane (2000) and J.Campbell (2000) for an excellent introduction and survey of current asset pricing
} methodologies. 


\section{Chapter 7: The downside of international equity markets}

The stochastic discount factor arises through uncertainty, uncertainty due to time and risk preferences. Time preference is simply the preference of $\$ 1$ today rather than $\$ 1$ tomorrow, so that foregoing $\$ 1$ today requires more than $\$ 1$ to be received tomorrow. If there is no uncertainty regarding the amount received, then the return is classed as riskfree. Although inflation risk still erodes the real purchasing power of the $\$ 1$. However if the return on the asset is uncertain, characterised by a probability distribution around the expected return, then the asset has an element of risk, and also needs to be discounted according to the extent of this risk.

This depends crucially on the degree of risk aversion exhibited by the investor, since an investor highly averse to risk will discount at a greater rate than would an investor less averse to risk. The difficulty in pricing financial assets arises through the choice of the correct discount factor. In order to provide models of general equilibrium assumptions are required to be made as to the behaviour of general investment behaviour.

Before we delve into the alternative approaches in determining the stochastic discount factor it is of interest to state an important inequality in the finance literature. This is derived using the mathematical identity for covariance:

$$
\operatorname{Cov}(M, X)=E(M X)-E(M) E(X)
$$

Now writing the difference between the return on the risky asset $\mathrm{i}$ and the risk free rate in terms of the stochastic discount factor in equation (7.2) and using the identity in equation (7.3) gives us the following equation (7.4): 


$$
\begin{aligned}
E_{t}\left[M_{t+1}\left(R_{i, t+1}-R_{f, t+1}\right]\right. & =0 \\
& =E_{t}\left(M_{t+1}\right) E_{t}\left(R_{i, t+1}-R_{f, t+}\right)+C\left(v M_{t+1}, R_{i, t+1}-R_{f}\right)_{t+1}
\end{aligned}
$$

Simply rearranging equation (7.4) gives us the expected risk premium of asset $i$ in terms of the covariance with the stochastic discount factor:

$$
E_{t}\left(R_{i, t+1}-R_{f, t+1}\right)=\frac{-\operatorname{Cov}\left(M_{t+1}, R_{i, t+1}-R_{f, t+1}\right)}{E_{t}\left(M_{t+1}\right)}
$$

Since the covariance between the stochastic discount factor and the assetsí risk premia is defined as equation (7.6), where $\tau$ is standard deviation and $\rho$ is correlation:

$$
\left.\operatorname{Cov}\left(M_{t+1} R_{i, t+1}-R_{f, t+1}\right)\right)=\rho\left(M_{t+1} R_{i, t+1}-R_{f, t+1}\right) \tau\left(R-R_{f}\right) \tau(M)
$$

And that correlation always lies between $\tilde{n} 1<\rho<1$, we know from the accounting identity in specified in equation (7.1) that the following inequality regarding the standard deviation of the stochastic discount factor must hold relative to the standard deviation of an assetsí risk premium. 


$$
\frac{E_{t}\left(R_{i, t+1}-R_{f, t+1}\right)}{\tau\left(R_{i, t+1}-R_{f, t+}\right)} \leq \frac{\tau\left(M_{t+}\right)}{E_{t}\left(M_{t+1}\right)}
$$

Equation (7.7) implies certain restrictions for the stochastic discount factor. It requires the ratio of the standard deviation of the SDF to the mean to be at least as great as the Sharpe ratio on an assetís risk premium - the expected risk premium divided by the standard deviation of the risk premium.

Models for asset pricing therefore focus on the definition of the SDF. Current theory in finance rests on the belief that the stochastic discount factor, M, can be captured by expected utility theory. Investors are thought to maximise utility by maximising consumption over time. The choice on how to optimally spread consumption over time therefore enables a model of investment to be derived, and provides the basics of finance theory as we know it today. This approach provides the basis to the modern theories currently dominating asset pricing, such as the Capital Asset Pricing Model (CAPM) and Arbitrage Pricing Theory (APT). However it is the use of the consumption based approach to asset pricing and the premise that investors maximise consumption over time that leads to the puzzles surrounding the size of the risk-return trade off in financial markets. We shall first look more deeply into the consumption-based approach to asset pricing before delving into the ensuing puzzles. 


\subsubsection{CONSUMPTION BASED APPROACH}

Modern portfolio theories of the CAPM and APT are derived using the consumption-based approach to asset pricing. In this framework general equilibrium models can be found under the axioms of Von Neumann and Morgensternís expected utility theory ${ }^{54}$. Investorsí utility is derived from consumption, whereby the utility function is both increasing (reflecting an individualsí increasing desire for more consumption) and concave (reflecting a declining marginal utility for additional consumption). This curvature depends on the exact degree of aversion to risk, as well as to the rate at which consumption is substituted between periods. It is fairly straightforward to derive the first order equation for optimal consumption and portfolio choice in terms of utility from consumption. Assuming a two-period model where consumption, $\mathrm{c}$, is equal to the original consumption level, e, minus the price $\mathrm{p}$, of an asset $\xi$, bought, we can write consumption at time $\mathrm{t}$ as in equation (7.8):

$$
c_{t}=e_{t}-p_{t} \xi
$$

Consumption available in the following period is determined by the consumption level in the following period, $\mathrm{e}_{\mathrm{t}+1}$ and the payoff, $\mathrm{x}$ at time $\mathrm{t}+1$ on the asset $\xi$. 


$$
c_{t+1}=e_{t+1}+x_{t+1} \xi
$$

Maximising utility over the two periods with respect to the asset enables us to find the first order condition for optimal consumption and portfolio choice:

$$
\frac{\partial u}{\partial \xi}=-p_{t} u^{\prime}\left(c_{t}\right)+E_{t}\left[\beta u^{\prime}\left(c_{t+1}\right) x_{t+1}\right]=0
$$

The asset price is maximised when the loss in utility from buying another unit of the asset equals the gain in discounted expected utility from the payoff of an additional unit of the asset at time $\mathrm{t}+1$. At this point the investor is indifferent to holding the asset for the next period and reaping the return and selling the asset for the price p. Rearranging (7.10) simply gives us an expression in the format of equation (7.1), so that we are able to determine the equation for the SDF.

$$
p_{t}=E_{t}\left[\beta \frac{u^{\prime}\left(c_{t+1}\right)}{u^{\prime}\left(c_{t}\right)} x_{t+1}\right]
$$

The stochastic discount factor, $\mathrm{M}$ in the consumption-based approach is therefore simply determined as:

54 The three the necessary and sufficient conditions for the existence of a state additive utility function in expected utility theory as formulated by Von Neumann and Morgenstern are the preference axiom, the substitution/independence axiom and the archimedean axiom. 


$$
M_{t}=\beta \frac{u^{\prime}\left(c_{t+1}\right)}{u^{\prime}\left(c_{t}\right)}
$$

Depending on the exact specification of the utility function, for example quadratic, exponential or power, alternative specifications for the relative risk aversion levels and the time preference parameters can be derived and tested against empirical observations. One of the most common specifications is the Capital Asset Pricing Model ${ }^{55}$, which can be derived in a two-period framework under quadratic utility or exponential utility and normality, or in a multi period framework under quadratic utility and i.i.d. returns or $\log$ utility and normality ${ }^{56}$. The CAPM results in the stochastic discount factor being a linear function of a single factor, the market portfolio, with risk represented by beta. Alternatively, multi-factor models such as Arbitrage Pricing Theory (APT) result in the stochastic discount factor being a linear function of multiple risk factors.

The risk-return puzzles in current finance theory arise through the assumptions behind the consumption-based approach to asset pricing. The puzzles are related to the concern of using consumption in order to determine the SDF, with empirical evidence suggesting that risk is under-estimated. The additional return made on equities is not justified by the extent of the extra risk involved in holding equities. This can only be captured in the consumption-based approach by high levels of risk aversion. Likewise international investment is also perceived as more risky than modern portfolio theory would suggest.

55 The CAPM was first introduced by Sharpe (1964), Lintner (1965) and Mossin (1966). 


\subsubsection{EQUITY PREMIUM PUZZLE}

Referring back to the inequality in equation (7.7) we can easily show this without the need to specify the precise nature of the utility function. To derive the extent of the equity premium puzzle we can simply look at the variances of the stochastic discount factor, $\mathrm{M}$, with that of consumption. Empirically over the last 100 years the premium for holding stocks in the US has been about $6 \%$ higher than for US Treasury bills ${ }^{57}$. Since the Treasury Bill rate is not very risky, and the riskfree rate of return is known ahead of time we can derive that the expected mean of the SDF is almost equivalent to the reciprocal of the riskfree rate from equation (7.3):

$$
1=E_{t}\left\lfloor M_{t+1} R_{f, r+1}\right\rfloor=E_{t}\left[M_{t+}\right] R_{f, r+1}
$$

This means that for the inequality in equation (7.7) to hold the volatility of the SDF must be in excess of the Sharpe ratio for the excess return on assets. Empirically this means an excess of around 0.5 for annual data or roughly 0.25 for quarterly data ${ }^{58}$. Since the volatility of consumption has empirically been extremely low with respect to market returns, implementing any of the mainstream utility functions in the consumption based approach therefore requires very high coefficients for risk aversion to raise the volatility of the SDF such that the inequality in equation (7.7) holds. Only with the risk aversion

\footnotetext{
See Cochrane (2000) for a concise derivation of the CAPM under various alternative assumptions.

See Kocherlakota (1996).

See Cochrane (2000), who uses the square root of time rule.
} 
parameter way in excess of realistic estimates can the inequality hold, as first derived by Mehra and Prescott who required a factor of 50 to fit the power utility model. This is the basis of the equity premium puzzle.

The puzzle is made worse through the low correlation of consumption with stock market returns. The inequality in equation (7.7) is bounded when this correlation is perfectly correlated, whereas less than perfect correlation requires the ratio of the expected SDF to the volatility of the SDF to be even greater. These bounds are the Hansen-Jaganathan bounds often used to test models of asset pricing. These can also be derived from the slope of the ray from the origin to the efficient frontier in the risk-return trade-off. Empirically the covariance of consumption growth to excess stock returns has been extremely low ${ }^{59}$.

Alternative theories to try to resolve this puzzle have therefore emerged. Kocherlakota (1996) provides a comprehensive survey of the recent developments in attempts to resolve the equity premium puzzle. Weil (1992) relaxes the assumption of complete markets with individuals being unable to insure themselves against fluctuations in labour income. The individual therefore faces additional risk from individual consumption growth, which is thought to raise the covariance between consumption and stock market returns. The impact of transaction costs, such as information costs, brokerage fees, load fees and the bid-ask spread, on reducing individuals ability to maximise the first order condition equation (7.10), is discussed by Aiyagari and Gertler (1991) and Heaton and Lucas (1995).

\footnotetext{
5 Campbell, Lo and MacKinlay (1997) estimate the covariance in log consumption growth to $\log$ excess returns in the US for annual data over the period 1889 to 1994 to be as low as 0.003 , requiring a risk-aversion coefficient of 19 to fit the power utility model.
} 


\section{Chapter 7: The downside of international equity markets}

However as Kocherlakota discusses, unfortunately none of the research into any of these areas has proved fruitful in adequately resolving the puzzle.

An alternative strand of the literature has focussed on alternative assumptions for investor preferences. Epstein and Zin (1989) and (1991) use a generalised expected utility formation of investor preferences, which moves away from the Von Neumann Morgerstern framework of expected utility theory. By using a recursive utility function they are able to separate the parameters for relative risk aversion and inter-temporal substitution, so that the restrictive case of one being the reciprocal of the other no longer is necessary. They use their approach (Epstein and Zin (1990) to try to resolve the equity premium puzzle; however their results only go part way in being able to explain the puzzle. Constantindes (1990) and Heaton (1995) both take on a habit formation approach to modeling investor preferences, with individuals who have consumed a lot in period ( $t-1)$ requiring greater consumption in period t to maintain the individualís level of utility. They provide some evidence which helps to partly reconcile the equity premium puzzle; however the puzzle is not completely resolved.

An alternative approach however, first adopted by Abel (1990) and Gali (1994), and later by Campbell and Cochrane (1995) does also partly resolve the puzzle by focussing also on the risk to per capita consumption. They propose that rather than deriving utility solely from individual consumption, individuals are also concerned with the overall level of consumption in society, and therefore also derive utility from per capita consumption. This 
approach, known as ëkeeping up with the Jonesesí, enables individuals to be highly risk averse to per capita consumption risk, rather than to their individual consumption risk, and therefore enables an alternative explanation for the equity premium puzzle. However as Kocherlakota discusses the fundamental puzzle still remains as to the high level of risk aversion to per capita consumption risk rather than to individual consumption risk.

Further work in this area has been undertaken, such as by Jorion and Goetzman (1999) as to the possibility of incorrect measurement of the equity premium by focussing on data from the US. Siegel and Thaler (1997) take an international stance and believe that the equity premium is not as inflated as once thought. Mehra (1997) in an attempt to understand the puzzle looks at the difference between investment goals of young and middle-aged workers, concluding that the constraint facing young investors to borrow on future earnings, results in a deficient demand for equity.

The equity premium puzzle regarding the risk-return trade-off within domestic markets still remains a puzzle under the consumption-based approach to asset pricing and expected utility theory. Indeed the further puzzle regarding the risk-return trade-off observed on international financial markets also remains a puzzle to financial economists. It is to this second puzzle, the phenomenon of the home bias that we now turn. 


\subsubsection{HOME BIAS PHENOMENON}

The US equity market comprises of roughly half of the global equity market; however almost $94 \%$ of US equity funds are devoted to domestic securities ${ }^{60}$. In a similar manner domestic securities are favoured worldwide by investors. Using modern portfolio theory and the consumption-based approach to asset pricing, portfolio diversification should be seen as highly desirable since investors are able to reduce the risk of their overall portfolio by spreading risk across various assets. The same argument would appear to render the diversification benefits of international asset allocation of particular appeal, with investors also being able to diversify across international financial markets. The lack of international diversification however has been a puzzle to financial economists worldwide with investors commonly devoting too great a proportion of their portfolio to their domestic market.

In both the CAPM and APT asset pricing models risk is captured solely by the use of single factor or multi-factor pricing models for risk, stemming from expected utility theory. Investors behave consistently to risk according to the Von Neumann and Morgernstern axioms. Attitudes to risk therefore are consistent over the whole domain of the utility function. This results in these models attributing the same attitude to risk on domestic markets as to international markets, and results in the same optimal allocation of assets regardless of the level of wealth or aversion to risk. Investors with higher aversion to

\footnotetext{
60 See French and Poterba (1991).
} 
risk hold a greater proportion of the portfolio at the riskfree rate whilst still allocating the same percentages into international assets.

The home bias could well be attributed to barriers on international investment ${ }^{61}$, through government restrictions regarding foreign capital flows, as well as to foreign taxes, political and transfer risk, and additional information and transaction costs. This gives the domestic investor a comparative advantage to the home market and can therefore explain the additional risk involved in investing internationally. This home bias phenomenon gives rise to the notion that the perceived risk from investing internationally is higher than the standard measure of risk in the consumption based approach to asset pricing suggests, assuming of course that investors behave rationally. However within the expected utility framework any alternative behaviour to risk regarding international investment is not captured in the utility function of the investor.

Advances in behavourial finance may however be able to help us to determine how investors perceive risk, and using some of the advance in the area we develop an alternative approach to assessing risk-return trade-offs in financial markets. Many of the advances center on the inadequacies behind some of the axioms on which expected utility theory is based. Rather than imposing a rigid framework for investor preferences behavioural finance allows for non-standard behaviour. This is driven by nonstandard preferences or irrational behaviour, which is often based on empirical findings. The ëkeeping up with the Jonesesí model for utility, for example depends more on the 
Chapter 7: The downside of international equity markets

behavioural approach to finance $\tilde{n}$ an area we now turn to for an alternative approach to asset pricing.

\subsubsection{BEHAVIOURAL FINANCE}

Empirical research has however shown various deviations from the expected utility framework and the consumption based approach. Research for example in the area of behavioural finance has been shown to contradict the axioms and assumptions of expected utility theory. The most commonly stated violation of the axioms is probably the Allais paradox (1953), which contradicts the substitution axiom of expected utility theory. Investors have been shown to overweight outcomes that are considered certain, relative to outcomes that are merely probable ${ }^{62}$. Kahneman and Tversky (1979) also show that investors differ between negative and positive domains, and thus treat gains and losses differently, with certainty increasing with respect to the aversiveness to losses as well as to the desirability of gains ${ }^{63}$. This means that counter to the risk aversion hypothesis the utility function is not concave everywhere. There is strong evidence that investors prefer to reduce the probability of a loss from $\mathrm{p} / 2$ to 0 by more than from $\mathrm{p}$ to $\mathrm{p} / 2$. Furthermore investors do not appear to be independent between events. This violates the proposition that prospects are determined solely by final state probabilities ${ }^{64}$. Individuals appear to perceive gains and losses relative to a reference point rather than as a final state of wealth

61 See for example Stulz (1981).

62 Kahneman and Tversky (1979) define this as the certainty effect.

63 Kahneman and Tversky (1979) define this as the reflection effect.

64 Kahneman and Tversky (1979) define this as the isolation effect. 
or welfare. These failings of expected utility theory led Kahneman and Tversky (1979) to develop Prospect Theory. In order to account for their empirical findings, they weigh the outcomes by a value function where gains and losses are defined as deviations from a reference point. Generally this is concave for gains and convex for losses, as well as steeper for losses than for gains.

It is the basic fundamental problems with the consumption based approach to asset pricing that motivate the use of alternative approaches to be found for determining the stochastic discount factor M. We therefore move away from expected utility theory and turn to an alternative approach to try to adapt some of the features of behavioural finance and prospect theory into asset pricing: the downside risk model.

\section{1. 3 DOWNSIDE RISK}

It would appear that the risk of investing in equities (domestic and international) must be larger than presently captured by finance theory so that the extent of the risk-return tradeoffs on financial markets can be explained without the need to resort to extortionate levels of relative risk aversion. Advances in behavioural finance lead us to suppose that investorsí attitudes change over the domain of the utility function, so that for example the risk from losses is larger than for gains. The downside risk approach focuses on the downside domain of the utility function, so that wealth is maximised subject to a constraint that the investorís wealth does not fall below a pre-specified level with a given confidence level. Taking such an approach provides us with an alternative framework with which to 


\section{Chapter 7: The downside of international equity markets}

analyse risk-return trade-offs in domestic and international financial markets, in line with many of the empirical findings in behavioural finance.

Investors are therefore concerned about additional downside risk from assets, over and above the standard measure for risk, the standard deviation of returns. The downside risk constraint depends crucially on the relative risk aversion of the individual investor, the time horizon over which the investor invests, as well as the investorís wealth. Arzac and Bawa (1977) show that this choice criterion is reasonable from an economic standpoint since it implies desirable attributes toward risk according to the Arrow (1971) ñ Pratt (1964) theory of risk aversion ${ }^{65}$, even though the criterion does not adhere to the axioms of continuity and independency.

In the following section we outline the downside risk model, and using empirical data on the G7 countriesí equity and bond markets, observe the risk-return trade-offs for the US domestic and international markets. We shall see how the risk-return differs from the consumption-based approach to asset pricing, and discuss the benefits of taking such an approach.

\subsection{OPTIMAL ASSET ALLOCATION UNDER DOWNSIDE RISK}

We apply the downside risk model from Campbell et al. (2000). The investorís budget constraint is defined as the following equation:

65 See Arzac and Bawa (1977) for a more elaborate discussion. 


$$
W(0)+B=\sum_{i=1}^{n} \gamma(i) P(i, 0)
$$

Where $\mathrm{W}(0)$ is the initial wealth, B is the amount of borrowing, and the investor chooses the fractions of asset $i, \gamma(i)$ to be invested at time 0 . The investor is assumed to allocate the assets in the portfolio and to choose the amount to borrow or lend such that the expected level of final wealth is maximised. Investor preferences in the safety first world are such that he or she wishes to be $\mathrm{c} \%$ confident that the final value of the portfolio at time $\mathrm{T}$ will not fall below a downside risk level. This is the downside risk constraint, with the desired level of Value-at-Risk, $\mathrm{VaR}^{*}$ denoting the downside risk level:

$$
\operatorname{Pr}\left\{W(T, p) \leq W(0)-V a \mathrm{R}^{*}\right\} \leq(1-c)
$$

Because we are also focussing on the risk of losses, our measure for risk depends on the downside only. Such an approach is also in fitting with the reflection principle of Kahneman and Tversky where the downside is weighed more heavily than the upside. The expected wealth from investing in portfolio $\mathrm{p}$ at the end of the investment horizon becomes:

$$
E_{0}(W(T, p))=(W(0)+B)(1+r(p))-B\left(1+r_{f}\right)
$$


Chapter 7: The downside of international equity markets

From equation (7.14) to (7.16) Campbell et al. (2000) derive that the optimal asset allocation occurs when equation (7.17) is maximised:

$$
p^{\prime}: \arg \max _{p} S(p)=\frac{r(p)-r_{f}}{\varphi(c, p)}
$$

Where $\mathrm{r}(\mathrm{p})$ and $\mathrm{rf}$ are the returns on the portfolio and the risk free rate respectively. The numerator is thus the risk premium and the denominator is the expression for risk and is defined as follows:

$$
\varphi(c, p)=W(0) r_{f}-V a R(c, p)
$$

The level of risk aversion depends crucially on the confidence level associated with the investorís downside risk constraint $\tilde{n}$ the higher the confidence level, the more risk averse the investor. This allows us to move away from some of the general utility specifications of the consumption based approach by re-defining risk aversion as a confidence level rather than as a parameter for relative risk aversion in the utility function.

One of the features, which the downside model is able to incorporate, is an investorís notion of regret. The investor assesses the risk from investment according to the downside risk of the asset allocation with reference to the value the initial wealth would have attained if invested over the period at the risk-free rate. The investor therefore uses the riskfree rate of return as the benchmark with which he or she assesses the potential allocation strategy. This is very much in fitting with the behavioural response of investors, and in line with the habit formation model mentioned earlier. Risk is assessed relative to a 
benchmark, be it the average level of consumption or the deviation from the riskfree rate of return. Since the risk-return trade-off depends on the confidence level chosen by the investor, we are able to derive various mean-downside risk efficient frontiers for the various confidence levels, allowing a further dimension to be gained into modern portfolio management, and alternative insight to be gained into both the equity premium puzzle and the home bias phenomenon.

\subsubsection{DATA}

We use monthly data from the MSCI indices for the G7 countries in their home currencies: Canada, France, Germany, Italy, Japan, the UK and the US, as well as the Datastream 10Year Government Bond Index. The data is available from January 1975 until August 1998 for the MSCI indices and from January 1980 until August 1998 for the Datastream US 10Year Government Bond Index. We also use exchange rate data from the MSCI indices for the same period, so that we can use various numeraire countries. Summary statistics for the series are given in table 7.1 .

\subsubsection{EMPIRICAL RESULTS FOR US DOMESTIC MARKET}

To derive the risk-return trade-off for the US domestic market we optimise the downside risk model for portfolio combinations of the MSCI index and the 10-Year Government Bond index using equation (7.17). We see that the downside risk approach to asset allocation results in a larger risk-return trade off the greater the confidence level chosen in 


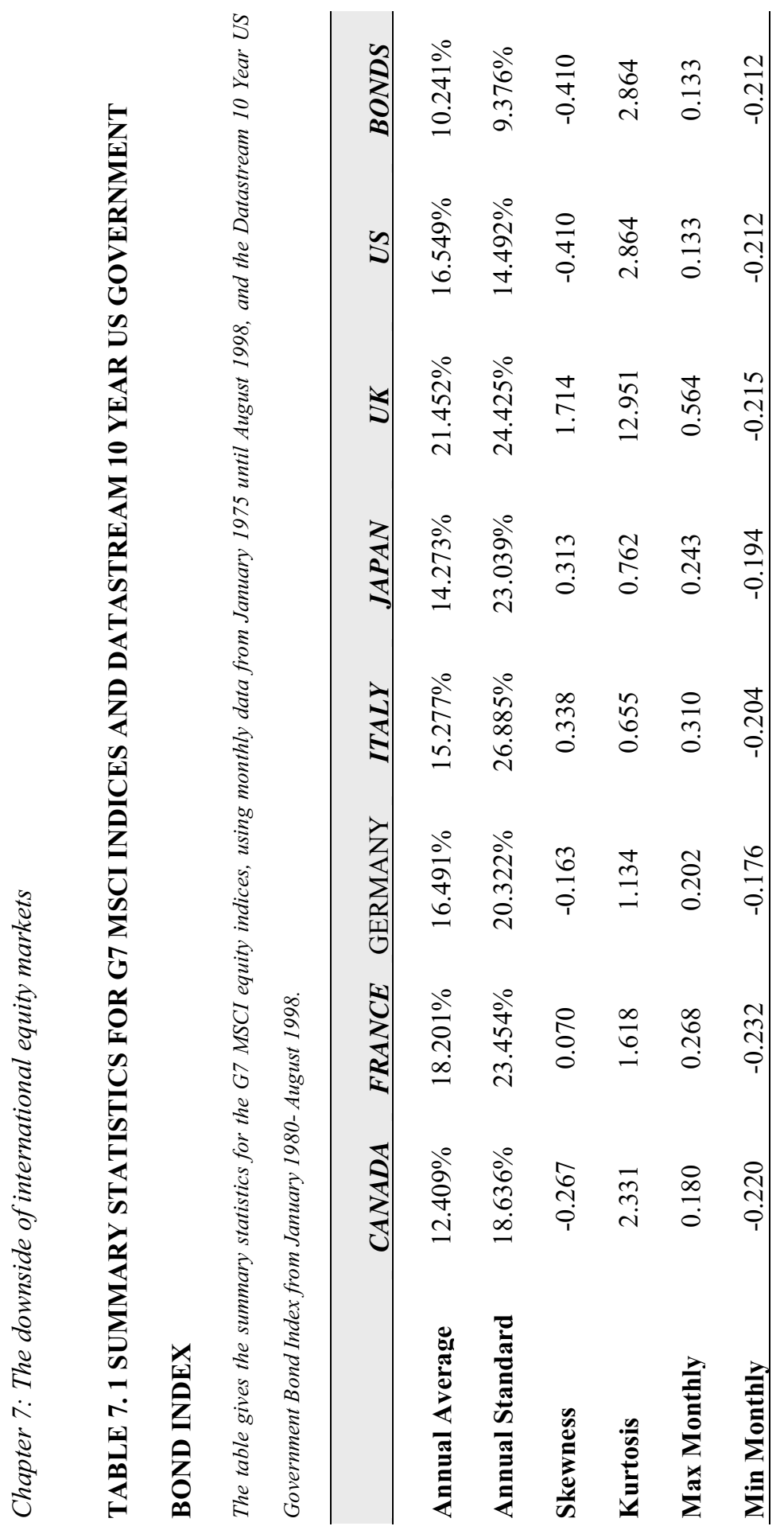


association with the downside risk constraint. This is shown by the efficient VaR frontiers in Figures $7.1 \& 7.2$ for the $95 \%$ and $99 \%$ confidence levels respectively.

\section{FIGURE 7.1 EFFICIENT VAR FRONTIER ñ MONTHLY VAR AT 95\%}

\section{CONFIDENCE LEVEL}

The figure presents the risk return trade off for portfolios of Stocks and Bonds whereby risk is measured by the downside risk measure $\varphi$ of the portfolio at the 95\% confidence level. The returns and VaR estimates are obtained using monthly data on the US MSCI Returns Index and the 10- Year Datastream US Benchmark Government Bond Index for the period January 1980 until August 1998. We present the efficient frontier for the empirical distribution and the parametric normal approach.

\section{Efficient VaR Frontier: 95\% Confidence Monthly Data January 1980 - August 1998}

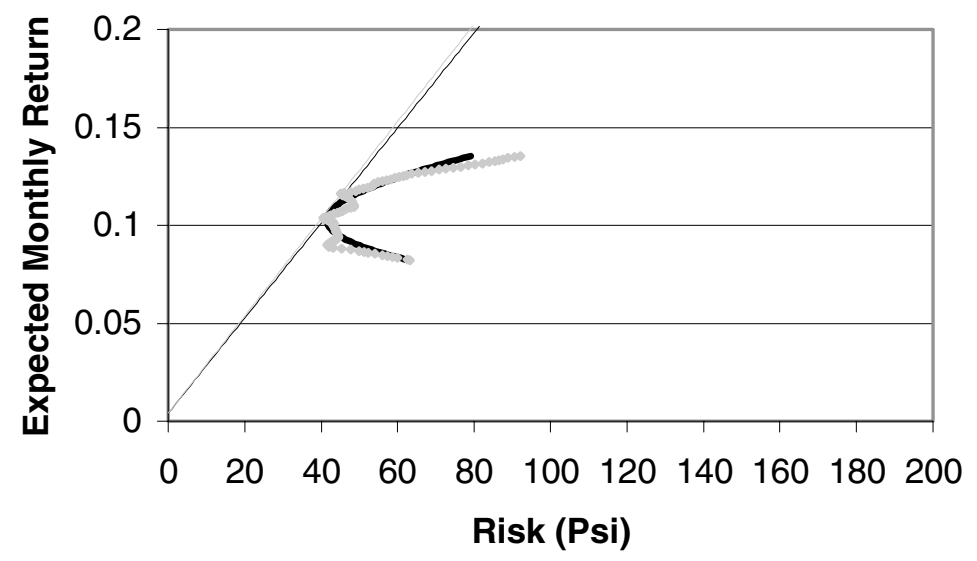

\begin{tabular}{|ll|}
\hline Historical & Normal \\
SML Normal & SML Empirical \\
\hline
\end{tabular}


Chapter 7: The downside of international equity markets

FIGURE 7.2 EFFICIENT VAR FRONTIER ñ MONTHLY VAR AT 99\%

\section{CONFIDENCE LEVEL}

The figure presents the risk return trade off for portfolios of Stocks and Bonds whereby risk is measured by the downside risk measure $\varphi$ of the portfolio at the 99\% confidence level. The returns and VaR estimates are obtained using monthly data on the US MSCI Returns Index and the 10- Year Datastream US Benchmark Government Bond Index for the period January 1980 until August 1998. We present the efficient frontier for the empirical distribution and the parametric normal approach.

\section{Efficient VaR Frontier: 99\% Confidence Monthly Data January 1980 - August 1998}

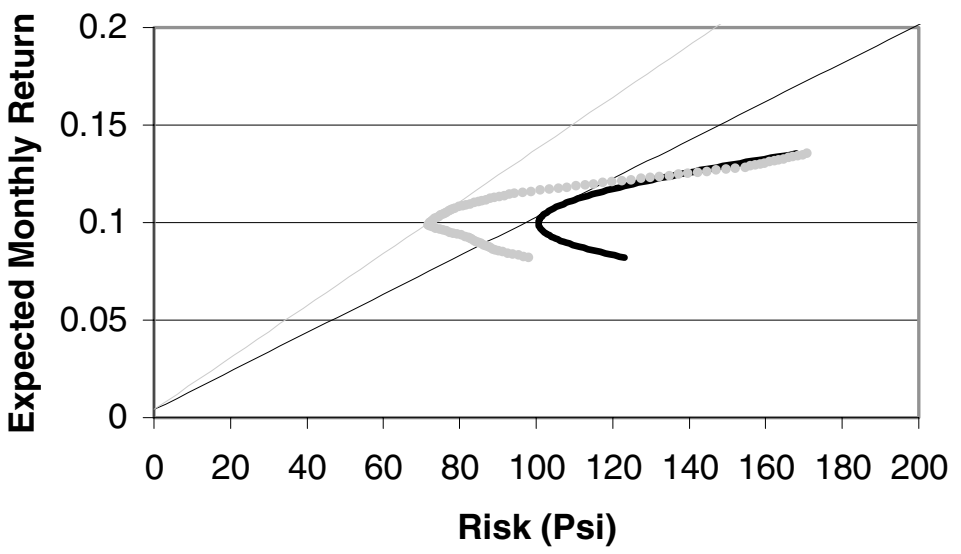

\begin{tabular}{|ll}
\hline Historical & Normal \\
SML Normal & SML Empirical
\end{tabular}

The efficient VaR frontier is similar to the mean-variance frontier, apart from the assumptions behind the definition of risk. Risk is defined in terms of the probability of downside loss relative to the riskfree rate and the confidence level defines the individualís 


\section{Chapter 7: The downside of international equity markets}

risk aversion. This results in an additional dimension being able to be captured through the use of the risk aversion level of the investor, which changes the risk-return trade off accordingly. The downside risk constraint also determines the decision on how much of the portfolio should be held at the risk free rate $\tilde{\mathrm{n}}$ the borrowing or lending decision; however the movement along the Security Market Line depends on the efficient VaR frontier. This contrasts with the expected utility theory approach whereby the optimal allocation is found regardless of the level of risk aversion, and only comes into play on the decision whether to borrow or lend. A movement along the Security Market Line determines this with the trade-off between risk and return remaining unaltered. This result is due also to the parametric assumption of normality, which is required for the meanvariance efficient frontier; however within the downside risk framework, the empirical distribution or a parametric distribution may be used.

Assuming the existence of a representative investor the model represents the general equilibrium in the economy. By focusing on the size of the risk premium of equities over the riskfree rate, we can then see from the efficient VaR frontier how the risk aversion level of the representative investor results in alternative trade-offs for risk and return. The greater the risk aversion of the investor, the less risk the investor is willing to take for a given level of return. This can be formulated alternatively as the higher the return required for taking on the same level of risk. It is in this manner that we are able to provide an alternative approach to assessing risk-return trade-offs in financial markets, and can provide an alternative justification as to why the return on equities has been so high relative to the risk $\tilde{\mathrm{n}}$ the presence of additional downside risk to the investor. In our analysis so far we have not needed to define the exact nature of the stochastic discount 


\section{Chapter 7: The downside of international equity markets}

factor and meet the inequality of equation (7.7). However it is certainly of interest to determine at exactly what confidence level general equilibrium is found in the economy, so that a general asset-pricing model in terms of the SDF can be determined. This depends on assumptions as to the risk averseness of the representative average investor, and assumptions to be made regarding intertemporal substitution. For a general equilibrium model we would need to specify more specifically how the downside risk constraint changes over time.

As we shall see from the data on international financial markets, the downside risk approach leads us to suspect that the representative investor concerned with monthly returns focuses on a confidence level of around $99 \%$ for the downside risk constraint. We now therefore turn to an empirical analysis of the risk-return trade-offs for international equity markets using the downside risk model.

\section{3 EMPIRICAL RESULTS FOR INTERNATIONAL EQUITY MARKETS}

Using the data on the MSCI indices we are able to derive how the downside risk model optimally allocates international equities with respect to various numeraire currencies. Starting with a domestic US investor we can see from Figure 7.3 that using the empirical distribution the US investor allocates a greater proportion of his or her portfolio in the domestic market as the confidence level associated with the downside risk constraint increases. If however the assumption of normality is assumed, the proportion held in the 
Chapter 7: The downside of international equity markets domestic market is constant, regardless of the confidence level chosen in association with the downside risk $^{66}$.

\section{FIGURE 7. 3 INTERNATIONAL DIVERSIFICATION FOR US INVESTORS}

The figure gives the optimal portfolio selection for an international equity portfolio in the G7 MSCI equity indices for a US investor. Optimal allocations are found using the empirical distribution, using monthly data from January 1975 until December 1998, and under the assumption of multivariate normality, for a variety of confidence levels.

\section{International Diversification US}

MSCI Indices January 1975 - December 1998

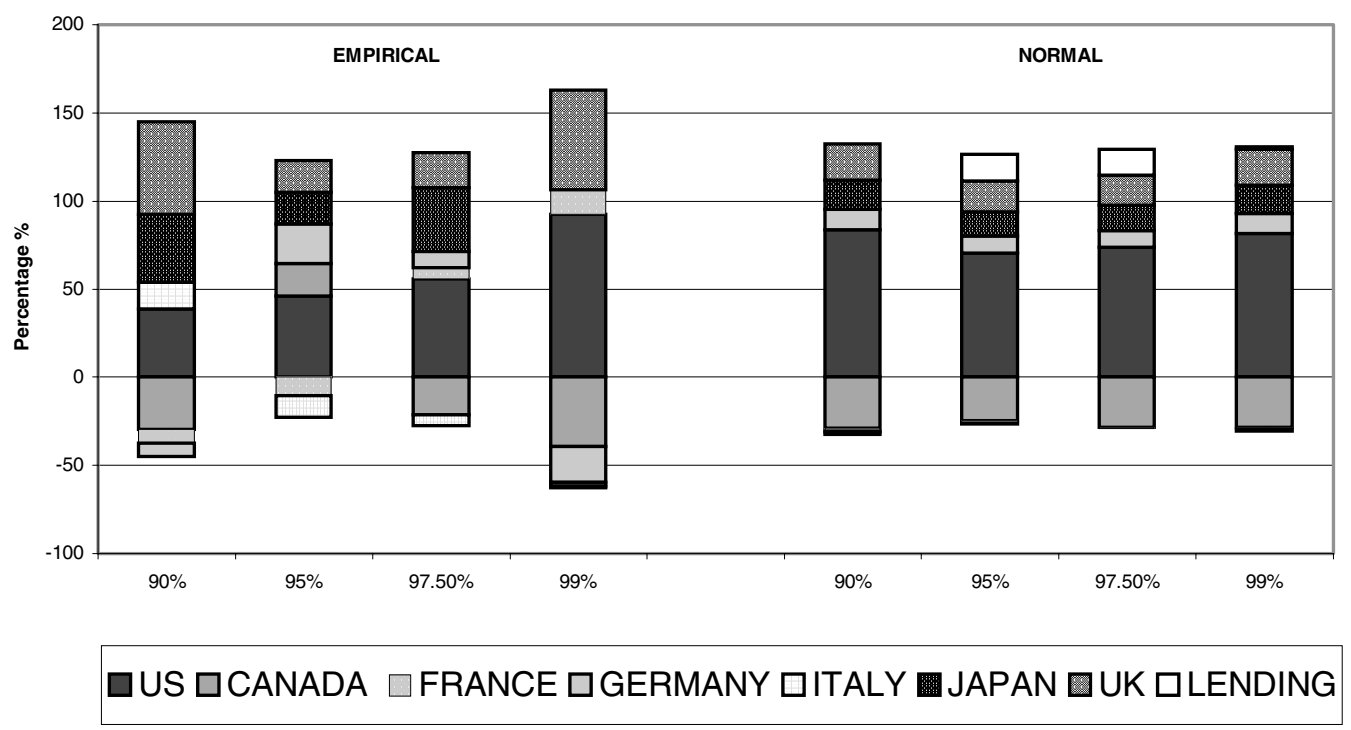

66 The result of a home bias is not affected by the imposition of short sale constraints. 
We compare the results from the empirical distribution using the downside risk model to those assuming bivariate normality for the model. This enables us to compare the results with those in a mean-variance world, whereby the parametric assumption of normality results in the optimum point occurring at the same point regardless of the confidence level $\operatorname{chosen}^{67}$. It is interesting to observe how the use of bivariate normality results in the international allocation being identical (only statistical error is incurred) whereas the home-bias phenomenon is captured by the use of the empirical distribution in the downside risk model. The figures for the US domestic investor are given in Table 7.2.

To explain the lack of international diversification the confidence level chosen by the investor must be greater, so that the risk aversion level of the representative investor has a confidence level of over $97.5 \%$. The optimal portfolio allocation is less into those assets with greater probabilities of extreme losses. Using the notion of regret, the regret is higher when investing abroad since the deviation from the benchmark is greater. Thus the confidence level with which the investor is concerned with is higher.

We also provide results for alternative numeraire currencies in Tables 7.3, 7.4 and 7.5, for UK, German, and Japanese investors.

67 See R. Campbell et al (2000) for a more detailed analysis of the use of parametric assumptions in the downside risk model. 


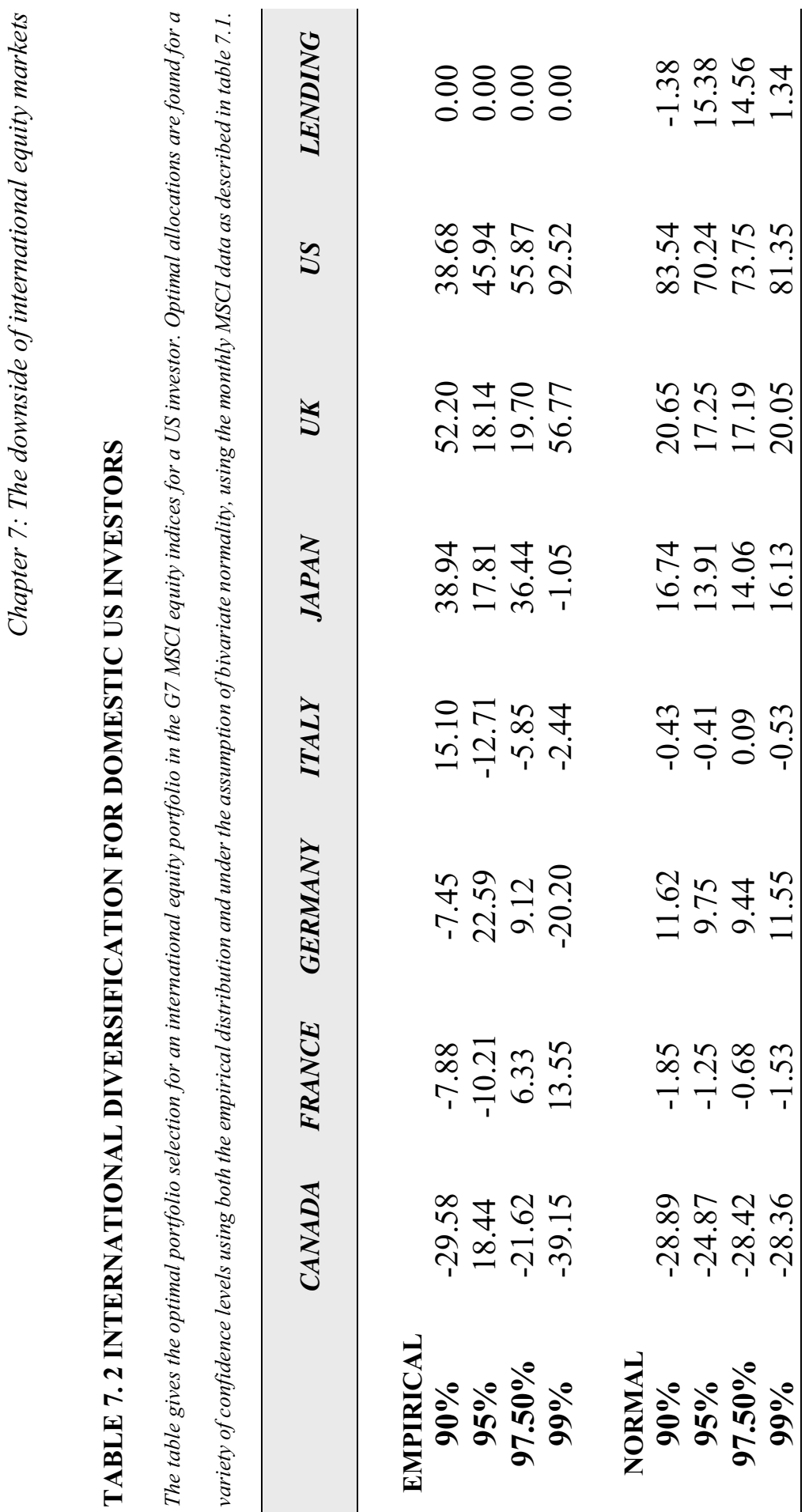



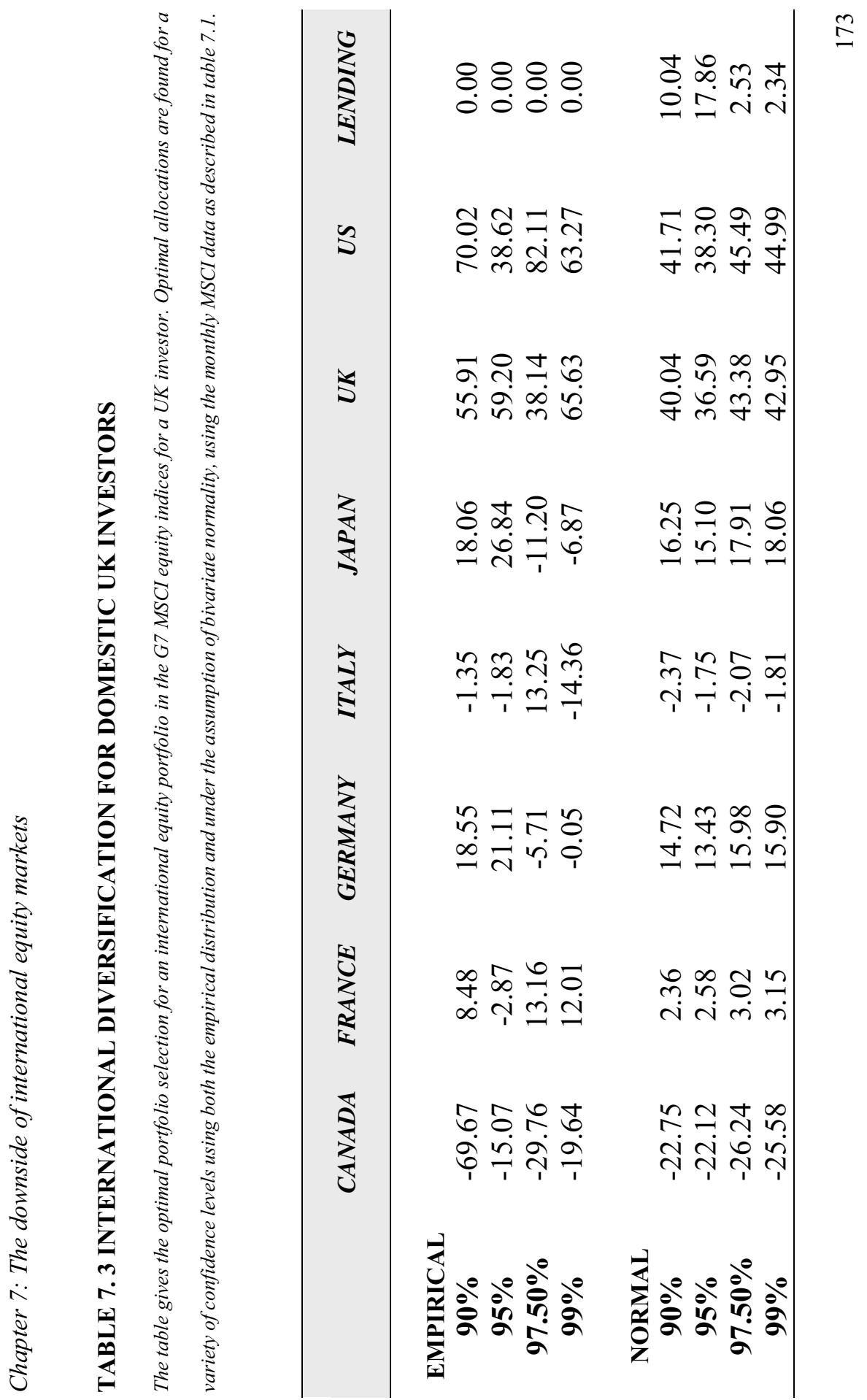

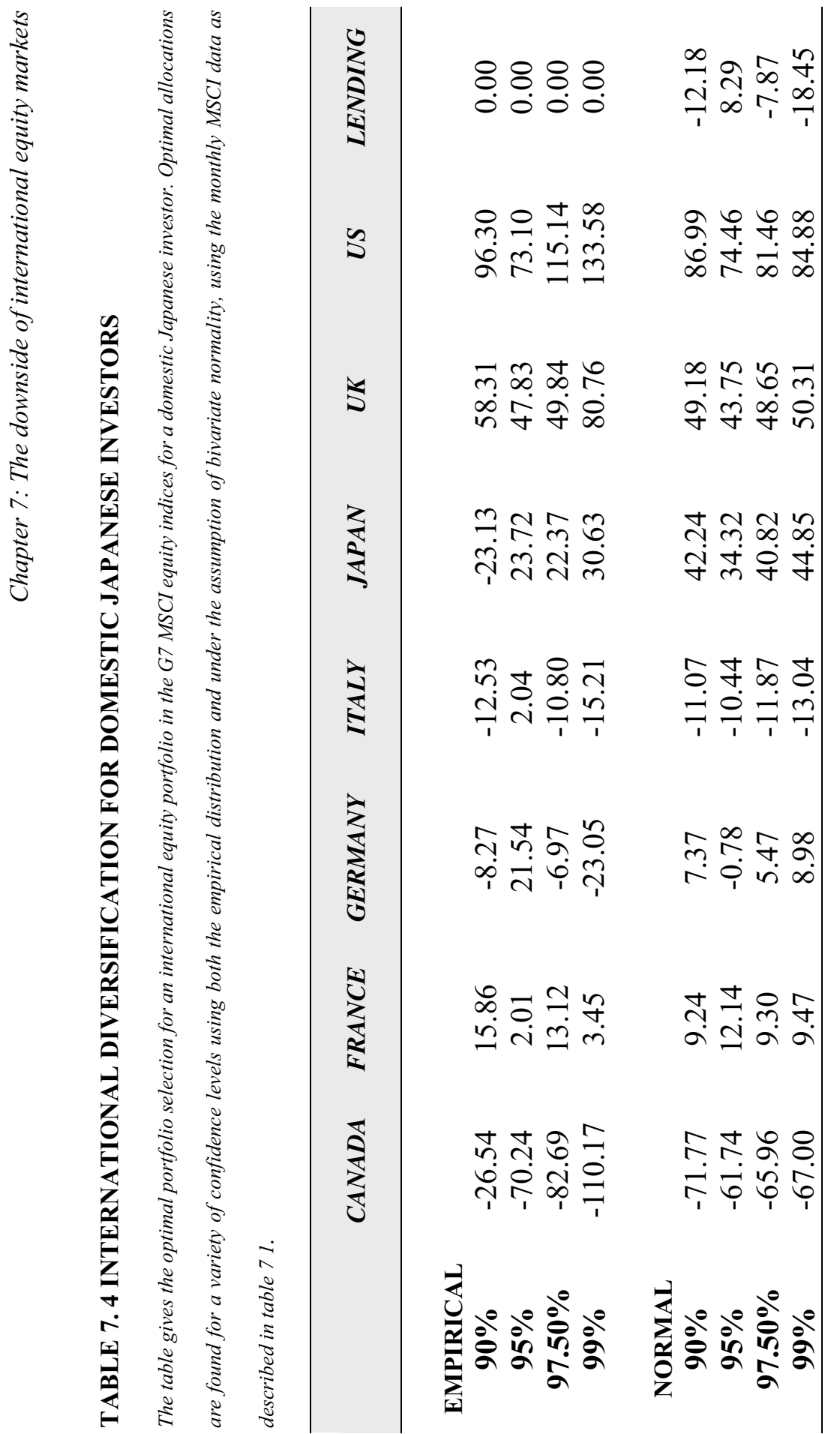


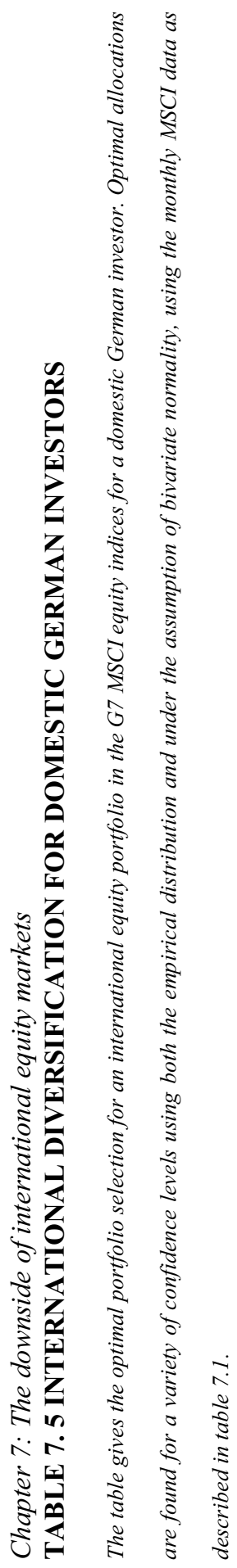

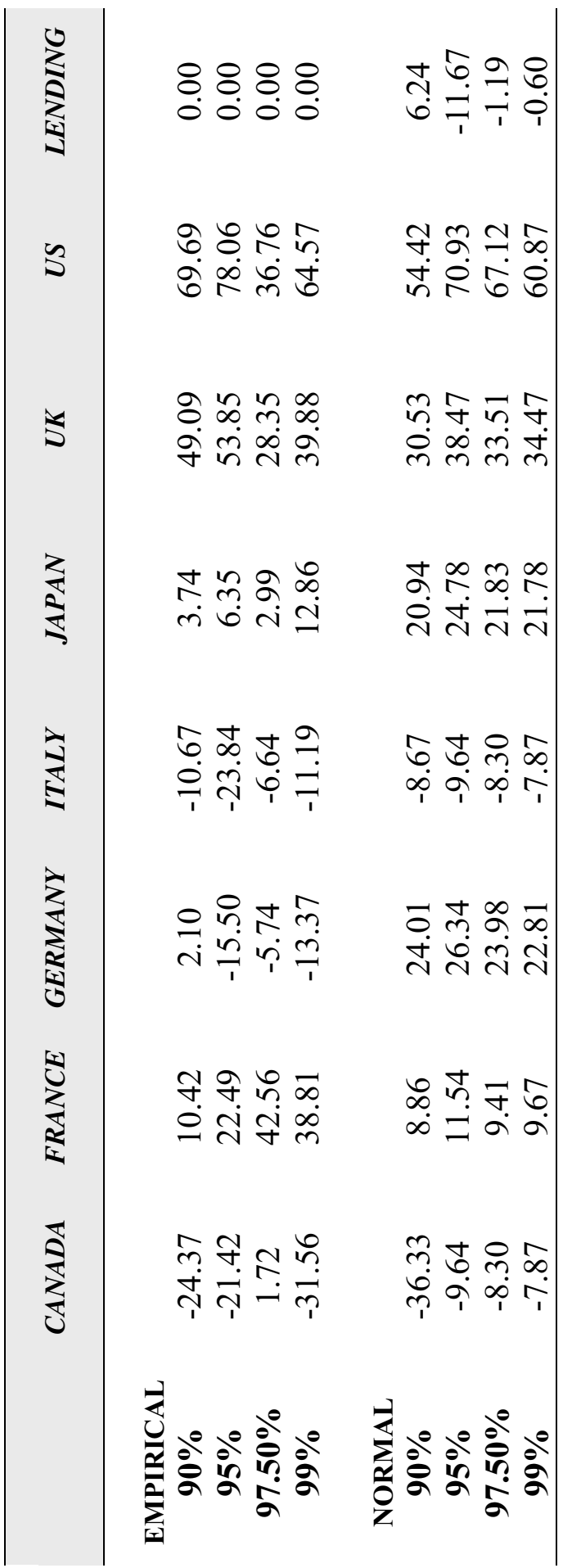


Chapter 7: The downside of international equity markets The downside risk model also provides evidence of a home bias in the UK, with the percentage held in the domestic market greater than that under the assumption of normality. However for Germany and Japan a smaller proportion of the domestic market is held, with German investors tending to have a greater tendency to short the market the greater the confidence level of the associated downside risk constraint. It could well be that the more volatile nature of the yen and the mark against the US dollar and their poor performance over the more recent years leads to greater downside risk of holding equities in both Japan and Germany, even for the domestic investor.

In the downside risk framework the impact of the correlation coefficient between markets is captured in the measure for risk. If correlation increases then the correlation coefficient becomes vital in the decision whether to hold international assets. Empirical research has however shown that as we move further into the tails of the distribution, where extreme events occur, we find that the correlation between international markets increases, and hence the benefits to diversification are reduced ${ }^{68}$. In the downside risk framework for optimal asset allocation we need not assume a constant coefficient for the joint distribution of returns, since we can use the empirical estimate for correlation for the associated confidence level, so the effects of increasing correlation coefficients are captured. It is probably the effect of increasing correlation in the left tail of the distribution which accounts for the changing empirical optimum.

68 See Butler and Joaquin (2000), who estimate that the occurrence of greater correlation in bear markets results in the Sharpe ratio being more than $50 \%$ too large, as well as Campbell, Koedjik and Kofman (2000), and Longin and Solnik (1999) who provide empirical evidence of increasing correlation in the left tail of the distribution. 


\section{Chapter 7: The downside of international equity markets}

This effect is not captured in the mean-variance framework, where correlation is assumed to be constant over the distribution, and no distinction is made between the associated confidence level of the investor. If it is the case that investors focus on a high confidence level, where correlation is higher between international financial markets, then the benefits to international diversification could be dramatically reduced. The results therefore indicate a rational explanation of the lack of international diversification and the phenomenon of the home bias, where investors are generally more worried about potential downside losses, rather than having to resort to very high levels of risk aversion.

The use of downside risk with an alternative measure of relative risk aversion results in an alternative approach to assessing risk-return trade offs in financial markets. The results are therefore of significant interest for further research in asset pricing and portfolio management, whereby it is of interest to specify the utility function for how a downside risk investor changes his or her specification of the confidence level for alternative time horizons. This would enable a direct test of the downside risk approach against the consumption-based approach in finance theory.

\section{3 CONCLUDING REMARKS}

In this chapter we investigate the use of downside risk, focussing on negative movements in stock markets for the assessment of risk, and see if the downside risk approach to asset allocation is able to provide some greater insight behind the puzzles in current finance 
Chapter 7: The downside of international equity markets theory. Using MSCI data for the G7 countries and US Government Bond returns we are able to express the risk-return trade-off in financial markets in an alternative way, shedding new light on the puzzle surrounding the size of the equity premium. We also find that contrary to the assumption of mean-variance portfolio analysis investors concerned with downside risk tend to hold a larger proportion of their portfolio in domestic equities the more averse to risk they become. The results highlight the greater downside risk of holding international equities without the need to assume exuberant levels for risk aversion. 


\section{CHAPTER 8 CONCLUDING THOUGHTS}

The collection of essays in this thesis aim to contribute to the existing literature and exemplify the importance of correctly characterising how we measure risk in international financial markets. The implications of rethinking how we currently look at risk in financial markets lend themselves to many areas of finance, and in doing so we hope to try to improve some of the existing applications to current finance theory.

In the second chapter we focus on the use of potential loss (downside risk) as the appropriate measure of how investors perceive risk in financial markets and develop a portfolio selection model. We are able to determine the optimal selection of assets to be held for an investor concerned with a particular level of risk, as measured by the potential loss function. Modern portfolio theory optimises the risk-return trade-off with risk 
Chapter 8: Concluding thoughts

measured as the degree of dispersion (variance) of the return distribution. The model developed here allows for an alternative specification for risk to be incorporated into the portfolio allocation decision, namely downside risk; so that for an acceptable level of downside risk the portfolio attaining the highest achievable return is formed. This approach allows us to gauge the investorsí appetite for risk (degree of risk aversion) as a confidence level associated with the potential loss, rather than having to resort to the specification of a utility function. It also enables alternative parametric distributions to be be used, so that for financial returns differing from normality an alternative distribution may be used. We show just how sensitive the portfolio decision is to the degree of risk aversion, the time horizon over which the investor is concerned, and to the choice of parametric distribution.

Indeed for symmetrical distributions which can be proxied by the gaussian normal, the variance is able to capture the total variation in the probability distribution of returns and hence the full amount of risk facing the potential investor. The downside risk measure of Chapter 2 is only then compatible with the variance as a measure for risk. Financial returns however tend to be skewed and leptokurtotic. This means that not only is the probability of positive returns occurring different from that of negative returns, but also that the probability of large movements is greater than captured under the assumption of gaussian normality. This results in the variance not being able to fully capture the riskiness of financial market returns, and is the main motivation for the use of an alternative risk measure to be used. In order to capture the true riskiness of the dispersion of returns we need a measure for risk that not only focuses on the downside of the distribution (potential losses rather than potential gains) but also on the additional probability mass associated 


\section{Chapter 8: Concluding thoughts}

with large losses occurring. Only if the true downside risk can be estimated can the riskreturn trade-off be accurately understood and used in financial risk management.

Chapter 3 focuses on how we can improve upon downside risk estimation for financial risk management, so that we can incorporate the occurrence of fat tails and skewness into the estimates for market risk. Market risk is commonly estimated using Value-at-Risk, a measure for the value of the portfolio which is in danger of being lost for a given confidence level. We develop a model VaR-x, which is shown to work well for a variety of international equity markets.

In Chapter 4, following on from the research on improving the risk estimates for market risk, we develop a similar approach for Credit-at-Risk modeling and for the pricing of credit risk derivatives. Credit risk includes the additional risk over market risk of the potential default probability of the counterparty. If credit spreads widen then the credit risk also becomes greater. Modeling this additional risk can also be improved upon when incorporating the fat tailed and skewed nature of credit spread distributions.

Risk management is especially crucial in times of crisis, and it is only then that financial risk management techniques are really stressed. In Chapter 6 we develop the VaR-x approach, using a time conditional approach for improved estimates of market risk during extremely volatile periods on international equity markets. We look at the Asian financial crisis to exemplify the need for such an approach, and find that the conditional VaR-x methodology provides robust results. Indeed when looking at European stock markets over 
Chapter 8: Concluding thoughts

the more volatile period of the previous decade the use of the conditional approach has provided significant improvements ${ }^{69}$.

Even though emerging markets, such as the Asian-Tiger markets have been characterised as highly risky their apparent low correlation with international markets renders them capable of achieving overall high returns through risk diversification. In theory the diversification benefits are thought to be huge; however investors have shied away from these markets in more recent times, with the potential for diversification appearing to be severely under-exploited. Another explanation for this phenomenon is that the correlation structure for large negative movements in international markets is different from the correlation between returns in more-normal market conditions. If the correlation indeed is under-estimated for large potential losses, then the benefits to diversification are significantly curtailed, and hence the risk of investing in these markets is also underestimated. In Chapter 6 we therefore focus on an alternative measure for size conditional correlation, and observe theoretical and empirical correlation structures for various international equity markets. We find that under the assumption of bivariate normality, the correlation structure does increase for more extreme movements in bear markets (times of falling markets). We also find that the assumption of constant correlation may however be kept if an alternative parametric distribution, such as the student-t, is used. The correlation of such a distribution is able to capture the greater simultaneity of large movements in international equity markets.

69 See Huisman, Koedijk and Pownall (1999). 


\section{Chapter 8: Concluding thoughts}

The implications of the essays in Chapters 2-6 are brought together in the final research chapter, Chapter 7. Using the methodology of the downside risk portfolio model and the greater correlation structure which is implied through the adoption of the empirical distribution, we are able to see how an investor concerned with downside risk would optimise his or her international equity portfolio. Indeed we find evidence that the riskreturn trade-off in international equity markets is greater than previously thought, which gives rise to an alternative explanation of the current conundrum of the so called equity premium puzzle. We find that not only do investors allocate a greater share of their portfolio in the domestic market, home bias, but also that in order to explain the risk-return trade-off observed empirically the average representative investor has to have a fairly low appetite for risk, high risk aversion.

The essays put together here not only focus on an alternative view of risk in financial risk management but also more importantly emphasise a need to rethink how current risk management affects much of mainstream finance. The risk-return trade-off lies at the heart of investment banking and international finance, however little attention has been paid to the correct measure of risk. The research within this thesis stemming from an alternative approach to measuring risk derived from a risk management framework therefore provides an integral framework within which financial institutions can improve portfolio analysis. The managerial implications involve fore mostly reformulating the risk-return trade-off using the downside risk approach. The use of a fatter tailed distribution provides a tradeoff more in line with that found using historical data, and hence provides a more prudent investment strategy in line with the appetite for risk, which investors appear to have. A further crucial point is the determination of an investorís aversion to risk. In order to gauge 
Chapter 8: Concluding thoughts this risk within the framework outlined in this thesis, then an investor needs only to be able to address his maximum level of loss as a percentage of his investment holding, such a figure is of relative ease to determine with the use of a few psychological questions. Indeed a lot easier to determine than an investors utility curve, which is the foundation of current mainstream applications in finance. This thesis therefore provides a number of paths for further research within both risk management and portfolio analysis. In particular in behavioural finance, applications for highly skewed distribution, as well as for derivatives and tech-stocks. Of course a researcherís work is never finished. Having had the opportunity to ërethink risk in international financial marketsí has enabled me to draw the conclusions presented in this thesis. It is my hope that they shall be of benefit to practitioners in the field of finance. 


\section{CHAPTER 9 REFERENCES}

Abel, A. B. (1990) ëAsset Pricing under Habit Formation and Catching Up with the Jonesesí, American Economic Review, May, Vol. 80. No. 2, 38-42.

Aiyagari, S. R. and M. Gertler (1991) ëAsset Returns with Transaction Costs and Uninsured Individual Riskí, Journal of Monetary Economics, June, Vol. 27, No. 3, 311-331.

Allais, M. (1953) ëLe Comportement de líHomme Rationnel devant le Risque, Critique des Postulats et Axiomes de líEcole Americaineí, Econometrica, 21, 503-546.

Ang, A. and J. Chen (2000) ì Asymmetric Correlations of Equity Portfolios,̂̂ Working Paper Columbia University.

Arrow, K. J. (1971) ëEssays in the Theory of Risk Bearingi, Markham, Chicago, IL.

Arzac, E. R. and V. S. Bawa (1977) ëPortfolio Choice and Equilibrium in Capital Markets with Safety-First Investorsí, Journal of Financial Economics, 4, 277-288. 
Chapter 9: References

Bain, L.J. and M. Engelhardt (1987), ëIntroduction to Probability Theory and Mathematical Statisticsí, Duxbury Press Boston.

Ball, C.A., and W.N. Torous (1999) ëStochastic Correlation Across International Stock Markets,í Working paper.

Barnett, V. (1976) èThe Ordering of Multivariate Data,í Journal of the Royal Statistical Society, Series A, 139, 318-354.

Basak, S. and A. Shapiro (1999). ëValue-at-Risk Based Risk Management: Optimal Polices and Asset Pricesí. Working Paper.

Basle Committee on Banking Supervision (1996), ëAmendment to the Capital Accord to Incorporate Market Risksí, Basle Report nr.24, BIS.

Bekaert, G., C. Erb, C. Harvey, and T., Viskanta (1998) ëDistributional Characteristics of Emerging Market Returns and Asset Allocationí, Journal of Portfolio Management, 24, 102-116.

Bekaert, G., and C. Harvey (1995) èTime-Varying World market Integrationí, Journal of Finance, Vol. L, No. 2, p 403-444.

Bollerslev, T. (1986) ëGeneralised Autoregressive Conditional Heteroskedasticityí, Journal of Econometrics, 31,307-327.

Bollerslev, T (1987) ëA Conditionally Heteroskedastic Time Series Model for Speculative Prices and Rates of Returní, The Review of Economics and Statistics, 69, 542-547.

Bollerslev, T., R. Engle, and J. Wooldridge (1988) ëA Capital Asset Pricing Model with Time Varying Covariances,í Journal of Political Economy, 96, 116-131. 
Chapter 9: References

Boothe, P. and D. Glassman (1987) ëThe Statistical Distribution of Exchange Rates: Empirical Evidence and Economic Implicationsí, Journal of International Economics, $22,297-320$.

Boyer, B.H., M.S. Gibson, and M. Loretan. (1999) ëPitfalls in Tests for Changes in Correlations,í International Finance Discussion Papers, Nr. 597, Board of Governors of the Federal Reserve Board.

Butler, K. C and D. Joaquin (2000), ëAre the Gains from International Portfolio Diversification Exaggerated? The Influence of Downside Risk in Bear Marketsí, Working Paper.

Campbell, J. Y, (2000) ëAsset Pricing at the Millenniumí, The Journal of Finance, vol. LV, No. 4, August 2000, 1515-1567.

Campbell, J. Y. and J. Cochrane (1995), ëBy Force of Habit: A Consumption-Based Explanation of Aggregate Stock market Behaviourí, NBER Working Paper 4995.

Campbell, J. Y, A. W. Lo and A. C. MacKinlay, (1997), ëThe Econometrics of Financial Marketsí, Princeton University Press, Second Edition.

Campbell, R. A., P. Eicholtz, and R. Huisman, (2000), ëBusts in House Pricesí, Working Paper, LIFE, Maastricht University and Erasmus University Rotterdam.

Campbell, R. A., R. Huisman and C. G. Koedijk (2001), ëOptimal Asset Allocation in a Value-at-Risk Frameworkí, Forthcoming in The Journal of Banking and Finance.

Campbell, R.A., C.S. Forbes, K.G. Koedijk and P. Kofman (2001) ì Diversification Meltdown or the Impact of Fat Tails on Conditional Correlation,î Working Paper, Erasmus University Rotterdam. 
Campbell, R.A., K.G. Koedijk and P. Kofman (2001) ì Increased Correlation in Bear Markets,î Forthcoming in The Financial Analysts Journal.

Christensen, M., K. B. Jensen, and S. Olesen. (1999) ëValue at Risk in Time-Varying Covariance Models,í Working paper Aarhus School of Business: Aarhus.

Cochrane, J. H. (2000), ëAsset Pricingí, Unpublished manuscript, University of Chicago.

Constantindes, G. M. (1990) ëHabit Formation: A Resolution of the Equity Premium Puzzleí, Journal of Political Economy, June 1990, Vol. 93, 3, 519-543.

Constantinides, G. M., J. B. Donaldson and R. Mehra (2000) ëJunior Canít Borrow: A New Perspective on the Equity Premium Puzzleí, Working Paper University of California.

Coval, J. D. and T. J. Moskowitz (1999), ëHome Bias at home: Local Equity Preference in Domestic Portfoliosí, University of Michigan Business School and University of Chicago Working Paper.

Das, D. and R. Uppal (1999) ëThe Effect of Systemic Risk on International Portfolio Choiceí. Working Paper.

Danielsson, J., and C. G. de Vries (1997) ëValue-at-Risk and Extreme Returnsí, Working Paper, London School of Economics.

Danielsson, J., and C. G. de Vries (1998) èThe Cost of Conservatism: Extreme Returns, Value-at-Risk and the Basle ëMultiplication Factorî, LSE Working Paper.

Economist Intelligence Unit (1998a) ëSecuring the Downside: A Proposal for Solving Financial Crisesí, Strategic Finance, June to August 1998. 
Chapter 9: References

Economist Intelligence Unit (1998b) ëHow Firms are Reacting to Turmoil in Asiaí, Strategic Finance, June to August 1998.

Engle, R. (1982) ëAutoregressive Conditional Heteroskedasticity with Estimates of the Variance of the United Kingdom Inflationí, Econometrica 50, p 391-407.

Engle, R., and K. Kroner (1995) ëMultivariate Simultaneous Generalized ARCH,í Econometric Theory, 11 (1995), 122-150.

Engle, R. F. and S. Manganelli (1997) ëCAViaR: Conditional Autoregressive Value at Risk by Regression Quantilesí University of California, Working Paper.

Epstein, L. G. and S. E. Zin (1989), ëSubstitution, Risk Aversion, and the Temporal Behaviour of Consumption and Asset returns: A Theoretical Frameworkí, Econometrica, Vol. 57, No. 4, July 1989, 937-969.

Epstein, L. G. and S. E. Zin (1990), ì First-orderí Risk Aversion and the Equity Premium Puzzleí, Journal of Monetary Economics, Vol. 26, 387-407.

Epstein, L. G. and S. E. Zin (1991), ëSubstitution, Risk Aversion, and the Temporal Behaviour of Consumption and Asset returns: An Empirical Analysisí, Journal of Political Economy, Vol. 99, No. 2, 263-286.

Erb, C.B., C.R. Harvey and T.E. Viskanta (1994) ëForecasting International Correlation,í Financial Analysts Journal, 50, 32-45.

Fama, E. F. and R. Roll (1968) ëSome Properties of Symmetric Stable Distributionsí, Journal of the American Statistical Association, 63, 817-846.

French, K. R. and J. M. Poterba (1991), ëInvestor Diversification and International Equity Marketsí American Economic Review, 81, 222-226. 
Gali, J. (1994) ëKeeping Up with the Joneses: Consumption Externalities, Portfolio Choice, and Asset Pricesí, Journal of Money Credit and Banking, Feb. Vol. 26, No.1, $1-8$.

Harlow, W. V. (1991) ëAsset Allocation in a Downside-Risk Framework,í Financial Analysts Journal, Vol. 47, September-October, 28-40.

Harvey, C. and A. Siddique (1999). ëConditional Skewness in Asset Pricing Testsí. Forthcoming in Journal of Finance.

Heaton, J. (1995) ëAn Empirical Investigation of Asset Pricing with Temporally Dependent Preference Specificationsí Econometrica, May 1995, Vol. 63, No. 3, 681717.

Heaton, J. and D. Lucus (1995) ëEvaluating the Effects of Incomplete Markets on Risk Sharing and Asset Pricingí Working Paper, Northwestern University.

Hendriks, D. (1996) ëEvaluation of Value-at-Risk Models Using Historical Dataí, Federal Reserve Bank of New York Economic Policy Review, April.

Hill, B. (1975) ëA Simple General Approach to Inference about the Tail of a distribution,í Annuals of Mathematical Statistics, 3, 1163-1174.

Huisman, R., C. G Koedijk, C. Kool, and F. Palm (1998) ėThe Fat-Tailedness of FX Returnsí, Working Paper, Limburg Institute of Financial Economics, Maastricht University.

Huisman, R., C. G Koedijk, C. Kool, and F. Palm, (2000) ëFat Tails in Small Samplesí, Forthcoming in the Journal of Business and Economic Statistics. 
Chapter 9: References

Huisman, R., C. G. Koedijk, and R. A. Pownall (1998) ëVaR-x: Fat Tails in Financial Risk Managementí, Journal of Risk, 1, Fall, 47-61.

Huisman, R., C. G. Koedijk, and R. A. Pownall (1999) ëDealing with Extremes: Introducing VaR-x to Financial Risk Managementí, Derivatives Weekly.

Jansen, D. W and C. G. de Vries (1991) ëOn the Frequency of Large Stock Returns: Putting Booms and Busts into Perspectiveí, The Review of Economics and Statistics, $73,18-24$.

Jansen, D.W., C. G. Koedijk, and C. G. de Vries (1998) ëPortfolio Selection with Limited Downside Riskí, LIFE Working Paper.

Jarrow, R.A. and S.M. Turnbull (1995) ëPricing Derivatives on Financial Securities subject to Credit Riskí, The Journal of Finance 50 No. 1 (March), 53-85.

Jarrow, R.A. and S.M. Turnbull (1998) ëCredit Riskí, in Alexander, Carol (Ed.) Risk Management and Analysis, Wiley, New York, 237-253.

Johnson, N.L., and S. Kotz (1972) ëDistributions in Statistics: Continuous Multivariate Distributionsí, John Wiley \& Sons, Inc.

Jorion, P. (1996) ëRisk²: Measuring the Risk in Value at Riskí, Financial Analysts Journal, November/December, 47-56.

Jorion, P. (1997) ëValue at Risk: The New Benchmark for Controlling Derivatives Riskí, McGraw-Hill.

Jorion, P. and W. N. Goetzman (1999) ëGlobal Stock Markets in the Twentieth Centuryíí The Journal of Finance, Cambridge; June 1999; Vol. 54, 3; 953-980. 
Chapter 9: References

J P Morgan Bank (1996) ëRiskMetrics Technical Manualí, New York: J P Morgan Bank, Fourth Edition.

Kahneman, D., and J. L. Knetsch and R. H. Thaler (1990) ëExperimental Tests of the Endowment Effect and the Coax Theoremí, Journal of Public Economics, 98, 13251350.

Kahneman, D. and M. Riepe (1998) íAspects of Investor Psychologyí, Journal of Portfolio Management, Summer, 52-65.

Kahneman, D. and A. Tversky (1979) ëProspect Theory: An Analysis of Decision under Riskí, Econometrica, Vol. 47, 263-291.

Kaminsky, G. L. and S. L. Schmukler (1998) ëWhat Triggers Market Jitters?: A Chronicle of the Asian Crisesí, World Bank Working Paper.

Karolyi, G. A. and R. M. Stulz (1996) ëWhy Do Markets Move Together? An Investigation of U.S.-Japan Stock Return Comovements,í Journal of Finance, 51, 951986.

Kendall, M. and A. Stuart (1977) ëThe Advanced Theory of Statisticsí, Charles Griffin \& Company Ltd.: Bucks, $4^{\text {th }}$ edition, Volume 1 (1977).

Kocherlakota, N. R. (1996) èThe Equity Premium: Itís still a Puzzleí, Journal of Economic Literature, Vol. XXXIV, March 1996, 42-71.

Koedijk, K. G., M. M. Schafgans and C. G. de Vries (1990) ėThe Tail Index of Exchange Rate Returnsí, Journal of International Money and Finance, 93-108. 
Chapter 9: References

Koedijk, K. G., P. A. Stork and C. G. de Vries (1992) ëDifferences Between Foreign Exchange Rate Regimes: The View from the Tailsí, Journal of International Economics, p 462-473.

Kupiec, P. H. (1995) èTechniques for Verifying the Accuracy of Risk Measurement Modelsí, The Journal of Derivatives, vol. 2 December, 73 ñ 84.

Leibowitz, M. L. and S. Kogelman (1991) ëAsset Allocation under Shortfall Constraintsí. Journal of Portfolio Management, Vol. 17, 18-23.

Linsmeier, T.J. and N.D. Pearson (2000) ì Value at Risk,î Financial Analysts Journal, March/April, 47-67.

Lintner, J. (1965) ëSecurity Prices, Risk and Maximal Gains from Diversificationí, Journal of Finance, 20, no. 4, 587-615.

Longin, F. (1997) ëBeyond the VaRí. ESSEC Working Paper.

Longin, F. and B. Solnik (1995) ëIs the International Correlation of Equity Returns Constant: 1960-1990?í Journal of International Money and Finance, February 1995, 14, 3-26.

Longin, F. and B. Solnik (2001) ì Extreme Correlation of International Equity Markets,î The Journal of Finance, 56, 2, 649-676.

Loretan, M. and W.B. English. (2000) ì Evaluating ëCorrelation Breakdownsí during Periods of Market Volatility,î International Finance Discussion Papers, Nr. 658, Board of Governors of the Federal Reserve Board. 
Chapter 9: References

Loretan, M. and P.C.B Phillips (1994) ëTesting the Covariance Stationarity of HeavyTailed Time Series: An Overview of the Theory with Applications to Several Financial Data Seriesí, Journal of Empirical Finance, 1, 211-248.

Lucas, A. and P. Klaassen (1998) ëExtreme Returns, Downside Risk, and Optimal Asset Allocationí, Journal of Portfolio Management, Vol. 25, 71-79.

Mandelbrot, B. (1963) èThe Variation of Certain Speculative Pricesí, Journal of Business, $36,394-419$.

Mehra, R. and E. Prescott (1985) èThe Equity Premium: a Puzzleí. Journal of Monetary Economics, March 1985, 145-161.

Mossin, J. (1966) ëEquilibrium in a Capital Asset Marketí Econometrica, 34, no. 4, 738783.

Pennings, J. M. and A. Smidts (2000) ëAssessing the Construct Validity of Risk Attitudeí, Management Science, Vol. 46, no. 10, 1337-1348.

Phoa, W. (1999) ëEstimating Credit Spread Risk Using Extreme Value Theoryí, The Journal of Portfolio Management, Spring, 69-73.

Pownall, R. A. and C. G. Koedijk (1999) ëCapturing Downside Risk in Financial Markets: the Case of the Asian Crisisí, Journal of International Money and Finance, Fall 1999, 853-870.

Pratt, J.W. (1964) ëRisk Aversion in the Small and in the Largeí Econometrica, Vol. 32, No. 1-2, 122-136.

Ramchand, L., and R. Susmel (1998) ì Volatility and cross correlation across major stock markets,î Journal of Empirical Finance, 5, 397-416. 
Regier, M. H. and M. A. Hamdan (1971) ëCorrelation in a Bivariate Normal Distribution with Truncation in Both Variables,í Australian Journal of Statistics, 13 (1971), 77-82.

Rogalski, R. J. and J. D. Vinso (1978) ëEmpirical Properties of Foreign Exchange Ratesí, Journal of International Business Studies, 9, 69-79.

Roy, A. D. (1952) ëSafety-First and the Holding of Assetsí, Econometrica, 20, 431-449.

Sharpe, W. (1964) ëCapital Asset Prices: A Theory of Market Equilibrium under Conditions of Riskí, Journal of Finance, 19, No.3, 425-442.

Sharpe, W. (1994) èThe Sharpe Ratioí, Journal of Portfolio Management, 21, 49-58.

Siegel, J. and R. Thaler (1997) ëAnomalies: The Equity premium Puzzleí, Journal of Economic Perspectives, Winter.

Stulz, R. M. (1981) ëOn the Effects of Barriers to International Investmentí Journal of Finance 36, 923-934.

Stutzer, M. (1998) ëA Portfolio Performance Index and its Implicationsí, University of Iowa, Working Paper.

Subrahmanyam, M., Y. Ho Eom, and J. Uno (1998) ëCredit Risk and the Pricing of Japanese Yen Interest Rate Swapsí, NYU-Stern working paper.

Taylor, S. (1986) ëModeling Financial Time Seriesí, John Wiley \& Sons Ltd.

Theodore, S. and E. de Bobard, (1999), ëBrave New Worldi, Credit Risk Special Report, April, 21 ñ 22. 
Chapter 9: References

Von Neumann, J. and O. Morgernstern (1944) ëTheory of games and Economic Behaviourí, Princeton: Princeton University Press.

Weil, P. (1992) ëEquilibrium Asset Prices with Undiversifiable Labour Income Riskí, Journal of Economic Dynamic Control, July/Oct. 1992, Vol. 16, No. 3-4, 769-790. 
CHAPTER 10

\section{SUMMARY IN DUTCH NEDERLANDSE SAMENVATTING}

De verzameling essays in dit proefschrift beoogt een bijdrage te leveren aan de bestaande literatuur door het belang te illustreren van het juist meten van risico in internationale financî̂le markten. De gevolgen van een andere kijk op risico in financî̂le markten strekken zich uit tot vele gebieden van de financî̂le economie. Door op een andere manier naar risico te kijken, hopen we enkele bestaande toepassingen van de financieringstheorie te kunnen verbeteren.

In hoofdstuk 2 richten we ons op het gebruik van potentieel verlies (downside risk) als maat voor hoe beleggers risico in financî̂le markten waarnemen en ontwikkelen we een portefeuille-selectie-model. We zijn in staat een optimale selectie effecten te bepalen voor een belegger met een bepaald risiconiveau, gemeten door de potentî̂le verliesfunctie. Moderne portefeuilletheorie optimaliseert de trade-off tussen risico en rendement waarbij 
Chapter 10: Summary in Dutch risico gemeten wordt als de variantie van de rendementsverdeling. In het model dat hier ontwikkeld wordt, is het mogelijk een alternatieve specificatie van risico te gebruiken bij het samenstellen van de beleggingsportefeuille, namelijk potentieel verlies; dit betekent dat voor een acceptabel risiconiveau de portefeuille met het maximaal haalbare rendement wordt gevormd. Deze aanpak maakt het mogelijk de mate van risico-aversie (appetite for risk) uit te drukken als een betrouwbaarheidsniveau behorend bij het potentieel verlies; het specificeren van een nutsfunctie is op deze manier niet nodig. Bovendien kunnen alternatieve parametrische verdelingen gebruikt worden, waardoor voor financî̂le rendementen die afwijken van de normale verdeling gekozen kan worden voor een alternatieve verdeling. We laten zien hoe gevoelig de portefeuillekeuze is voor de mate van risico-aversie, de tijdshorizon van de belegger en de keuze van de parametrische verdeling.

Voor symmetrische verdelingen die benaderd kunnen worden met de normale verdeling, is de variantie in staat de totale variatie van de kansverdeling van de rendementen uit te drukken, en dus het volledige risico waarmee een mogelijke belegger te maken krijgt. Alleen dan is de downside-risk-maatstaf uit hoofdstuk 2 vergelijkbaar met variantie als maat voor risico. Financî̂le rendementen hebben echter de neiging om scheef verdeeld en dikstaartig te zijn. Dit betekent niet alleen dat de kans op positieve rendementen verschilt van de kans op negatieve rendementen, maar ook dat er een grotere kans is op grote bewegingen dan de aanname van een normale verdeling impliceert. Dit leidt ertoe dat het risico van financî̂le rendementen niet volledig uitgedrukt wordt in de variantie, en dit is de belangrijkste reden om een alternatieve maatstaf voor risico te gebruiken. Om het risico van de spreiding van rendementen op een juiste manier uit te drukken, hebben we een 


\section{Chapter 10: Summary in Dutch}

maatstaf nodig die zich niet alleen concentreert op de onderkant van de verdeling (potentî̂le verliezen in plaats van potentî̂le winsten) maar ook op de extra kansmassa behorend bij grote verliezen. Alleen wanneer het werkelijke downside risico geschat kan worden, is een juist begrip en gebruik van de trade-off tussen risico en rendement in financieel risicomanagement mogelijk.

Hoofdstuk 3 concentreert zich op de vraag hoe we schattingen van downside risico voor financieel risicomanagement kunnen verbeteren, zodanig dat we het optreden van dikke staarten en scheefheid van de verdeling kunnen opnemen in schattingen voor marktrisico. Een gebruikelijke methode om marktrisico te schatten is door middel van Value-at-Risk, een maat voor de waarde van de portefeuille die verloren kan gaan bij een zeker betrouwbaarheidsniveau. We ontwikkelen een model, VaR-x, dat goed blijkt te werken voor diverse internationale aandelenmarkten.

Voortbordurend op het onderzoek naar verbetering van schattingen voor marktrisico, ontwikken we in hoofdstuk 4 een soortgelijke aanpak voor Credit-at-Risk modellen en voor het prijzen van kredietrisico-derivaten. Kredietrisico is het risico van default van de tegenpartij, dat nog boven op het marktrisico komt. Als credit spreads groter worden, neemt het kredietrisico ook toe. Dit additionele risico kan beter worden gemodelleerd als rekening gehouden wordt met de dikke staarten en scheefheid van de verdelingen van credit spreads.

Risicomanagement is in het bijzonder van belang in tijden van crisis en vooral dan wordt er veel aandacht besteed aan financieel risicomanagement-technieken. In hoofdstuk 6 
Chapter 10: Summary in Dutch

ontwikkelen we de VaR-x aanpak verder, waarbij we gebruik maken van een tijdsafhankelijke benadering van marktrisicoschattingen gedurende extreem volatiele perioden op internationale aandelenmarkten. De noodzaak van een dergelijke aanpak wordt geÔllstreerd aan de hand van de Azî̂ crisis; de conditionele VaR-x methodologie geeft robuuste resultaten. Ook als we kijken naar Europese aandelenmarkten gedurende het volatielere deel van het vorig decennium, heeft de conditionele benadering significante verbeteringen opgeleverd ${ }^{70}$.

Zelfs al worden opkomende markten, zoals die van de Aziatische Tijgers, als zeer risicovol beschouwd, zijn deze door de lage correlatie die zij vertonen met internationale markten toch geschikt om door middel van diversificatie hoge overall rendementen te behalen. Volgens de theorie zouden de voordelen van diversificatie enorm zijn; de laatste tijd zijn deze markten echter gemeden door beleggers waardoor de mogelijkheden van diversificatie niet voldoende uitgebuit lijken te worden. Een andere verklaring voor dit fenomeen is dat de correlatiestructuur voor grote negatieve bewegingen in internationale markten verschilt van de correlatie tussen rendementen onder ënormalereí marktomstandigheden. Als de correlatie bij grote potentî̂le verliezen inderdaad wordt onderschat, dan zijn de voordelen van diversificatie kleiner en dus de risicoís van beleggen in deze markten groter. In hoofdstuk 6 concentreren we ons daarom op een alternatieve mat voor correlatie die afhangt van de grootte van de rendementen (size conditonal correlation) en bekijken we theoretische en empirische correlatiestructuren voor diverse internationale aandelenmarkten. We vinden dat onder aanname van een bivariate normale

\footnotetext{
70 Zie Huisman, Koedijk en Pownall (1999).
} 


\section{Chapter 10: Summary in Dutch}

verdeling, de correlatiestructuur inderdaad toeneemt bij extreme bewegingen in dalende markten (bear markets). We vinden ook dat de veronderstelling van een constante correlatie gehandhaafd kan blijven wanneer een alternatieve parametrische verdeling, zoals de student-t, gebruikt wordt. De correlatie van een dergelijke verdeling is in staat de grotere gelijktijdigheid van grote bewegingen in internationale aandelenmarkten uit te drukken.

De implicaties van de essays in hoofdstuk 2-6 worden samengebracht in het laatste hoofdstuk van het onderzoek. Door gebruik te maken van de methodologie van het downside risk-portefeuillemodel en de grotere correlatiestructuur die wordt geÔmpliceerd door toepassing van de empirische verdeling, kunnen we zien hoe een belegger die kijkt naar downside risico zijn of haar internationale aandelenportefeuille zou optimaliseren. We vinden inderdaad bewijs voor een grotere trade-off tussen risico en rendement in internationale aandelenmarkten dan voorheen aangenomen werd, wat een alternatieve verklaring voor het raadsel van de zogenaamde equity premium puzzle zou kunnen zijn. We vinden dat beleggers niet alleen een groter deel van hun portefeuille in de thuismarkt beleggen, home bias, maar ook dat om de empirisch gevonden trade-off tussen risico en rendement te verklaren, de gemiddelde belegger een vrij hoge risico-aversie moet hebben.

De essays die hier verzameld zijn concentreren zich niet alleen op een alternatieve kijk op risico in financieel risicomanagement maar, belangrijker nog, benadrukken ook de noodzaak om opnieuw na te denken over hoe het huidige risicomanagement een groot deel van de gangbare financiering beÔnvledt. De trade-off tussen risico en rendement is essentieel in investment banking en internationale financiering, ookal wordt er weinig 
aandacht besteed aan het correct meten van risico. Het onderzoek in dit proefschrift, dat voortkomt uit een alternatieve benadering van het meten van risico, afgeleid uit een risicomanagement kader, verschaft daarom een volledig kader waarbinnen financî̂le instellingen hun portefeuille-analyse kunnen verbeteren. De implicaties voor de praktijk houden in de eerste plaats een herformulering in van de trade-off tussen risico en rendement door gebruik te maken van de downside-risk-benadering. Het gebruik van een verdeling met dikkere staarten geeft een trade-off die meer in lijn is met de trade-off die in historische data wordt gevonden, en levert dus een voorzichtigere beleggingsstrategie op, in lijn met de mate van risico-aversie die beleggers blijken te hebben. Een ander cruciaal punt is het vaststellen van de mate van risico-aversie van een belegger. Om dit risico uit te drukken binnen het kader dat in dit proefschrift is weergegeven, hoeft een belegger slechts in staat te zijn zijn maximale verlies uit te drukken als een percentage van zijn investering. Dit getal is relatief gemakkelijk vast te stellen aan de hand van enkele psychologische vragen. Het is zeker een stuk gemakkelijker te bepalen dan de nutscurve van een belegger, waarop de huidige gangbare toepassingen in de financiering zijn gebaseerd. Daarom biedt dit proefschrift een aantal wegen voor verder onderzoek in zowel risicomanagement als portefeuille-analyse. In het bijzonder in behavioural finance zijn er toepassingen voor zeer scheve verdelingen, maar ook voor derivaten en technologie-aandelen. Natuurlijk is het werk van een onderzoeker nooit afgerond. De kans die ik gekregen heb om op een andere manier naar risico in financil̂le markten te kijken, heeft mij in staat gesteld tot de conclusies te komen die in dit proefschrift gepresenteerd zijn. Ik hoop dat ze van nut zullen zijn voor de financî̂le praktijk. 
Chapter 10: Summary in Dutch 
Chapter 10: Summary in Dutch 
Curriculum Vitae

\section{CURRICULUM VITAE}

Rachel Campbell (nee Pownall) was born on 28 December 1973 in Torquay, England. She attended Kingsbridge Comprehensive School, studying Maths, Economics and German to A-level. In June 1996 she obtained a first class BA degree in International Economics and Econometrics (German) from the University of Kent at Canterbury, England, which involved spending a year at the Phillips University, Marburg, Germany. In October 1997 Rachel obtained a Masters in International Economics from Maastricht University in The Netherlands, specialising in Econometrics and Finance. She joined the Finance and Accounting department in Maastricht in December 1997, where she was employed by the NWO to study for a Ph.D. in international finance. In January 1999 she moved to the Financial Management department at the Erasmus University in Rotterdam, The Netherlands, where she continued with her Ph.D. In October 1999 she was appointed Assistant Professor of Financial Risk Management within the department. Rachel has published her research in The Journal of Banking and Finance, The Journal of International Money and Finance, Financial Analysts Journal, The Journal of Risk, and Derivatives Weekly. Rachel is currently involved in further research, as well as recently becoming Managing Director of TheFinanceReader.com, an online company set up in January 2001 in partnership with Finedge BV and RiskTec, to help bridge the knowledge gap between academia and business. 




\title{
ERASMUS RESEARCH INSTITUTE OF MANAGEMENT
}

\author{
ERIM PH.D. SERIES \\ RESEARCH IN MANAGEMENT
}

Title:

Author:

Promotor(es):

Defended:

Series number:

Published:

ISBN:

Title:

Author:

Promotor(es):

Defended:

Series number:

Published:

ISBN:

Title:

Author:

Promotor(es):

Defended:

Series number:

Published:

ISBN:

Title:

Author:

Promotor(es):

Defended:

Series number:

Published:

ISBN:

\section{Operational Control of Internal Transport}

J. Robert van der Meer

Prof. dr. M.B.M. de Koster en Prof. dr. ir. R. Dekker September 28, 2000

1

ERIM Ph.D. series Research in Management

90-5892-004-6

\section{Quantitative Models for Reverse Logistics}

Moritz Fleischmann

Prof. dr ir J.A.E.E. van Nunen, Prof. dr ir R. Dekker, dr R. Kuik

October 5, 2000

2

Lecture Notes in Economics and Mathematical Systems, Volume 501, 2001, Springer Verlag, Berlin, 3540417117

\section{Optimization Problems in Supply Chain Management} Dolores Romero Morales

Prof. dr ir J.A.E.E. van Nunen, dr H.E. Romeijn

October 12, 2000

3

ERIM Ph.D. series Research in Management

90-9014078-6

\section{Layout and Routing; Methods for Warehouses}

Kees Jan Roodbergen

Prof.dr.ir. M.B.M. de Koster, Prof. dr ir J.A.E.E. van Nunen May 10, 2001

4

ERIM Ph.D. series Research in Management 90-5892-005-4 





\section{Rethinking Risk in International Financial Markets}

Rethinking Risk in International Financial Markets This thesis aims to address many of the issues raised concerning the appropriate definition and measurement of risk. An alternative approach to the estimation of risk, and the risk-return trade-off in international financial markets is investigated. Rather than focusing on the deviation of returns as the appropriate measure for risk, the more relevant negative domain when defining risk is focused upon. The notion of downside risk is applied as a more appropriate measure for risk. The focus is on a variety of international financial markets and applications of downside risk are used for improving market risk and credit risk management models. A downside risk approach for portfolio management is also derived, providing a pragmatic approach to implementing an alternative risk measure into international finance.

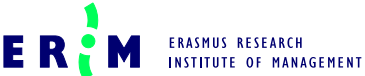

The Erasmus Research Institute of Management (ERIM) is the Research School (Onderzoeksschool) in the field of management of the Erasmus University Rotterdam. 